\title{
Coupled thermo-fluid-mechanical peridynamic model for analysing composite under
}

\section{fire scenarios}

\author{
Yan Gao, Selda Oterkus*
}

Department of Naval Architecture, Ocean and Marine Engineering, University of Strathclyde, Glasgow G4 0LZ, UK

\begin{abstract}
This study presents a coupled thermo-fluid-mechanical peridynamic model to predict composite responses under fire scenarios. Three-layer laminated composite is modelled by using ordinary state-based thermo-mechanical peridynamics. Both the mechanical deformation and the heat conduction are simulated by considering thermal degradation properties of composites. The fire is simulated as a heat-conducting fluid by using a thermo-fluid model based on the peridynamic differential operator. The ISO temperature-time curve is utilized to present the fire-induced high temperature. The composite damages due to direct contact with fire and due to hot gas induced by fire are investigated.
\end{abstract}

Keywords: composites, fire, fluid, thermal fluid-structure coupling, peridynamic, peridynamic differential operator

\section{Nomenclature}

\begin{tabular}{|c|c|c|c|}
\hline \multicolumn{2}{|c|}{ Composite material parameters } & \multicolumn{2}{|c|}{ Fluid parameters } \\
\hline$c_{v}$ & Specific heat capacity & $c_{f}$ & Specific heat capacity of fluid \\
\hline$C_{\text {convection }}$ & $\begin{array}{l}\text { Coefficient for natural } \\
\text { convection }\end{array}$ & $c_{\text {sound }}$ & $\begin{array}{l}\text { Artificial speed of sound of } \\
\text { fluid }\end{array}$ \\
\hline$E_{1}$ & $\begin{array}{l}\text { Elastic modulus in fibre } \\
\text { direction }\end{array}$ & $k_{f}$ & Fluid thermal conductivity \\
\hline$E_{2}$ & $\begin{array}{l}\text { Elastic modulus in } \\
\text { transverse direction }\end{array}$ & $P_{f}$ & Fluid pressure \\
\hline$E_{m}$ & $\begin{array}{l}\text { Elastic modulus of matrix } \\
\text { material }\end{array}$ & $S^{*}$ & $\begin{array}{l}\text { Non-dimensional Sutherland's } \\
\text { constant }\end{array}$ \\
\hline$G_{12}$ & Major shear modulus & $\alpha_{d f, 0}$ & $\begin{array}{l}\text { Initial value of fluid thermal } \\
\text { diffusivity }\end{array}$ \\
\hline$G_{m}$ & $\begin{array}{l}\text { Shear modulus of matrix } \\
\text { material }\end{array}$ & $\beta_{f}$ & $\begin{array}{l}\text { Thermal expansion coefficient } \\
\text { of fluid }\end{array}$ \\
\hline$G_{I C}$ & $\begin{array}{l}\text { Critical strain energy release } \\
\text { rate for first failure mode }\end{array}$ & $\gamma_{f}$ & $\begin{array}{l}\text { Ratio of specific heat capacity } \\
\text { of fluid }\end{array}$ \\
\hline
\end{tabular}

*Corresponding Author: Selda Oterkus, Department of Naval Architecture, Ocean and Marine Engineering, University of Strathclyde. Email: selda.oterkus@strath.ac.uk. 


\begin{tabular}{|c|c|c|c|}
\hline$G_{I I C}$ & $\begin{array}{l}\text { Critical strain energy release } \\
\text { rate for second failure mode }\end{array}$ & $\mu_{f}$ & Fluid viscous coefficient \\
\hline$k_{1}$ & $\begin{array}{l}\text { Thermal conductivity in } \\
\text { fibre direction }\end{array}$ & $\rho_{f}$ & Fluid density \\
\hline$k_{2}$ & $\begin{array}{l}\text { Thermal conductivity in } \\
\text { transverse direction }\end{array}$ & $\operatorname{Pr}$ & Prandtl number \\
\hline$k_{m}$ & $\begin{array}{l}\text { Thermal conductivity of } \\
\text { matrix material }\end{array}$ & $\mathrm{Ra}$ & Rayleigh number \\
\hline$K_{m}$ & $\begin{array}{l}\text { Bulk modulus of matrix } \\
\text { material }\end{array}$ & \multicolumn{2}{|c|}{ General and peridynamic parameters } \\
\hline$Q_{11}, Q_{22}, Q_{12}, Q_{66}$ & $\begin{array}{l}\text { Reduced stiff matrix } \\
\text { parameters }\end{array}$ & $g$ & $\begin{array}{l}\text { Magnitude of the gravitational } \\
\text { acceleration }\end{array}$ \\
\hline$\alpha_{1}$ & $\begin{array}{l}\text { Thermal expansion } \\
\text { coefficient in fibre direction }\end{array}$ & $t$ & Time \\
\hline$\alpha_{2}$ & $\begin{array}{l}\text { Thermal expansion } \\
\text { coefficient in transverse } \\
\text { direction }\end{array}$ & $T$ & Temperature \\
\hline$\alpha_{m}$ & $\begin{array}{l}\text { Thermal expansion } \\
\text { coefficient of matrix } \\
\text { material }\end{array}$ & $\mathbf{x}$ & Point position \\
\hline$\alpha_{d s 1}$ & $\begin{array}{l}\text { Thermal diffusivity in fibre } \\
\text { direction }\end{array}$ & $\mathbf{v}$ & Velocity \\
\hline$\alpha_{d s 2}$ & $\begin{array}{l}\text { Thermal diffusivity in } \\
\text { transverse direction }\end{array}$ & $\mathbf{u}$ & Displacement \\
\hline$\alpha_{d s m}$ & $\begin{array}{l}\text { Thermal diffusivity of } \\
\text { matrix material }\end{array}$ & $\mathbf{b}$ & Volumetric body force \\
\hline$\varepsilon$ & Surface emissivity & $\delta$ & $\begin{array}{l}\text { In-plane peridynamic horizon } \\
\text { size }\end{array}$ \\
\hline$\mu_{m}$ & $\begin{array}{l}\text { Lamé constant of matrix } \\
\text { material }\end{array}$ & $\hat{\delta}$ & $\begin{array}{l}\text { Horizon size in interlayer } \\
\text { normal direction }\end{array}$ \\
\hline$\rho_{s}$ & Density of composite & $\tilde{\delta}$ & $\begin{array}{l}\text { Horizon size in interlayer shear } \\
\text { direction }\end{array}$ \\
\hline$v_{12}$ & Major Poisson's ratio & $\Delta t$ & Time step size \\
\hline$v_{21}$ & Minor Poisson's ratio & $\Delta x$ & Initial point spacing \\
\hline$\sigma_{1 t}$ & $\begin{array}{l}\text { Tensile strength in fibre } \\
\text { direction }\end{array}$ & $\left({ }_{s}\right)$ & $\begin{array}{l}\text { Subscript denotes the } \\
\text { composite }\end{array}$ \\
\hline$\sigma_{\text {stefan-boltzmann }}$ & Stefan Boltzman constant & $\left({ }_{f}\right)$ & Subscript denotes the fluid \\
\hline
\end{tabular}

\section{Introduction}

The composite material response under fire load is a complex research topic since multidisciplinary fields are involved [1], e.g. thermodynamics, fluid mechanics, solid mechanics including composite material mechanics, chemical reaction theory, etc.[2]. 
Therefore, it would be a challenging task to numerically simulate this phenomenon by considering all the aspects.

Regarding the modelling of fire, understanding the mechanism and predicting the behaviour of fire is important and meaningful [3]. The fire can be considered as a summation of numerous flames [4]. Therefore, the flame can be treated as a basic element of fire. For the investigation of flame, the following fields are involved: the fluid field, the thermal field, the chemical reaction theory, and the species transport process.

- Fluid field: the flame can be categorized as a laminar flame or a turbulent flame. In laminar flames, the burning gas flows in a regular pattern, which is contrary to the turbulent flames [3]. For laminar flames, the Navier-Stokes equation can be directly adopted by numerical methods to simulate their behaviours. On the other hand, the large eddy simulation (LES) method is usually utilized for a turbulent flame [5].

- Thermal field: the heat transfer and chemical reaction heat generation are the main two aspects in the numerical thermal model. Enthalpy is considered a primary variable in a heat transfer process. While the Arrhenius Law is usually employed in the heat generation due to chemical reaction [6].

- Chemical Reaction: the reaction model should represent the reaction mechanism. Besides, the chemical kinetics of the elementary reactions [4] and their rates, e.g. chemical time scale, should also be decided [4]. A popular way to simulate the chemical reaction process is to use the commercial software Chemkin III [7] or the open-source software Cantera developed by Python [8].

- Species transport: The flame is a multi-phase and multi-component fluid flow [9]. Consequently, a mixture fluid model which can predict the species diffusion process is required if all the solid components are considered [10].

As it is introduced above, the flame can be classified as a multi-component, multi-phase, chemically reactive, and heat transfer fluid flow [11]. As a result, the flame mechanism involves the knowledge of thermodynamics, thermochemistry, chemical kinetics, and fluid mechanics, etc. [12]. In other words, the mass transfer, heat transfer, momentum transfer, and species transfer exist in the flame motion.[13] Since the governing equations are often too complicated for analytical solutions, the technique of numerical simulation is widely applied [3]. There are various numerical methods for flame modelling, i.e. finite element method (FEM) [14], finite volume method (FVM) [15], finite difference method (FDM) [16]. For these grid- 
based approaches, the space discretization should be fine enough for this complex combustion system. On the other hand, because of the presence of high gradients of physical variables in the flame, special attention should be paid to time integration stability [15]. The computation time and memory requirements should be taken into consideration. On the contrary to the above grid-based approaches (FEM, FVM, FDM), Lagrangian methods can predict the non-linear convection term exactly [17]. Direct Lagrangian methods have been applied to simulate the reacting flows [18-20]. The remeshed smoothed particle hydrodynamics (rSPH) in [17] is applied for the chemical reacting flows. A hydrogen/air opposed-jet burner numerical simulation is conducted with considering the Mach number effect [17]. The current existing numerical flame models cannot capture all the aforementioned aspects because of fire's complicated physical and chemical processes [3]. In the current study, the fire model is simplified as a heat-conducting single-phase fluid flow, ignoring the chemical reaction process and the diffusion of the components.

As to the investigation of composite response under fire loads which has been extensively studied [21-25], the key difficulty to the modelling is the complexity of the thermal, chemical, physical, and failure processes which control the composite structure behaviour [26]. The firing process of composites can be concluded as follows [26]:

- Thermal process: the heat conduction in composite materials due to fire-induced temperature change [27], heat generation from decomposition and charring[28], convective heat loss, etc.

- Chemical process: viscous softening, melting, decomposition, and char-fibre reactions, etc.[1].

- Physical process: thermal expansion and contraction, matric cracking, fibre-matrix interface debonding, softening, delamination damage, etc.

- Failure process: charring, pores creation (creation of gas from resin decomposition) [29], thermal degradation of elasticity properties, micro-cracking, and delamination, etc.[30].

The current numerical models cannot capture all composites' responses as explained above. Therefore, simplification is applied in the numerical simulations [31]. For most researches regarding the composite response in fire scenarios, the decoupling of fire from composites is widely adopted in many simulation approaches [32]. The fire is simply treated as a controlled heat flux or temperature boundary condition. The dynamics of fire are usually ignored. However, in the current study, the basic dynamics of fire motion, i.e. the thermal fluid flow, 
are considered during the composite response simulation. As a result, the physical problem of composite materials under fire belongs to the field of thermal fluid-structure interaction. The thermal degradation properties of composite due to high temperature [33] are considered. Furthermore, both matrix damage and fibre damage are taken into consideration.

In this study, as a novel numerical approach, peridynamics (PD) is utilised. PD is originally developed for predicting damages in isotropic materials [34, 35]. Then, the formulation is extended for modelling damage in composite materials [36-49]. The original form of PD is also extended for predicting thermal damages for both isotropic [50-53] and composite materials [54-58]. Moreover, PD is also used for predicting corrosion and oxidation damage for both isotropic and composite materials [50, 56, 59-62]. Therefore, due to the prediction capability of PD in many different applications, in this study PD is used to predict the composite damage under fire scenarios. The PD theory can be categorized as bond-based, ordinary state based and non-ordinary state based peridynamics [34, 35, 47]. According to bond-based PD theory, the PD force density vectors are equal in magnitude and parallel to the relative position vector. The Poisson's ratio is fixed as $1 / 3$ for two-dimensional and $1 / 4$ for three-dimensional isotropic materials [34, 35, 47]. Similarly, for laminated composite materials the Poisson's ratio is limited to $1 / 3$ for bond based PD theory $[48,63]$. Therefore, the four independent composite material parameters $\left(E_{1}, E_{2}, G_{12}, v_{12}\right)$ will reduce to two $\left(E_{1}, E_{2}\right)$ when the bond-based PD is used $[48,63]$. According to ordinary state based PD theory, the PD force density vectors are parallel to the relative position vector [64-68]. On the other hand, the PD force densities in nonordinary state based PD theory are in arbitrary directions [69-73]. Hence, the limitation on the Poisson's ratio in bond-based PD theory is removed [34, 35, 47]. In this study, ordinary state based PD theory is used for modelling laminated composites. For modelling fluid flow, peridynamic differential operator (PDDO) is used [74-76]. Consequently, higher-order derivatives existing in the Navier-Stokes equations are approximated by using integrations with the corresponding higher-order PDDO [74-76]. Therefore, in this study the PDDO based fluid model developed in $[77,78]$ is used to represent the fire flow model.

This study is organized as follows. The PD composite model which considers the thermal degradation properties is described in Section 2. Besides, the fire-induced temperature-time curve is provided to represent the fire effect in the thermal field. The PD thermo-fluid model and PD composite thermal model in a non-dimensional form are briefly reviewed in Section 3. Section 4 describes the numerical implementations. Then, Section 5 describes the numerical 
simulations. In this section, composite damages due to direct contact with fire and composite damages due to hot gas induced by fire are investigated. Finally, the conclusion is provided in Section 6.

\section{PD thermomechanical composite model under fire}

In this section, the temperature profile of fire and the thermal degradation properties of the composite material are provided. Subsequently, the PD composite model including these thermal properties is presented.

\subsection{Temperature profile of fire}

The fire temperature profile as a function of time is used to represent the fire effect. In many thermal modelling approaches, fire is simulated as a controlled heat flux or a temperature boundary condition $[26,79]$, which brings simplification for numerical simulations. Therefore, in the current study, the ISO 834 standard temperature-time curve [80] is utilized to represent the temperature profile of fire as

$$
T(t)=20+345 \log (8 t / 60+1)
$$

where $T$ represents the temperature in ${ }^{\circ} \mathrm{C}$ and $t$ represents the time in $\mathrm{s}$. The temperature plot is provided in Fig. 1.

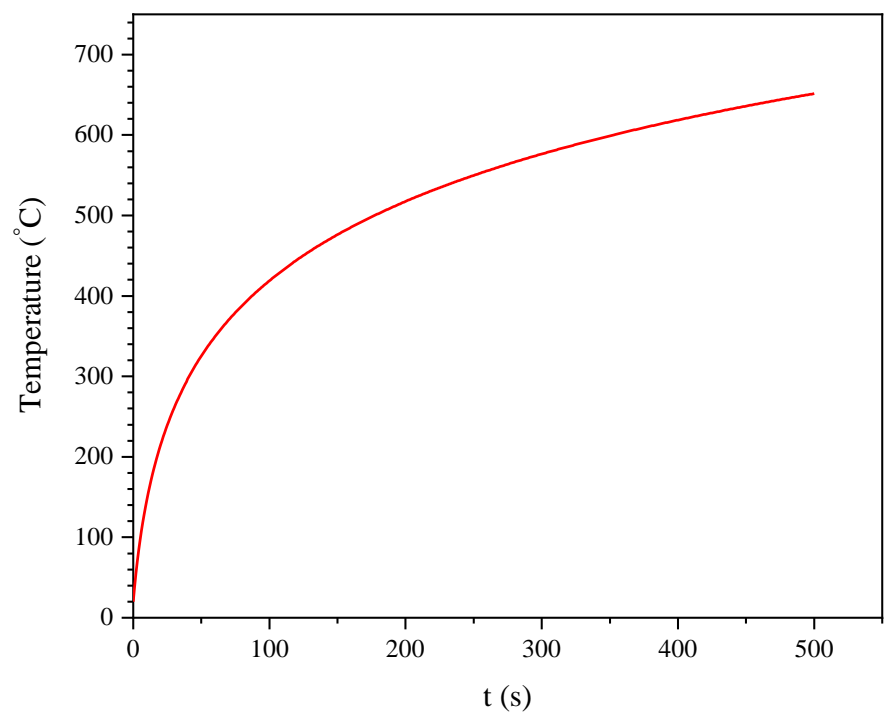

Fig. 1 ISO temperature-time curve representing fire [80] 


\subsection{Material degradation of composite material caused by fire}

In the current study, a carbon fibre-epoxy composite material is considered. The material properties are considered as functions of temperature since material softening will occur under high temperature conditions. The thermal degradation properties from [81] are utilized and incorporated into the PD model. Regarding the material thermal properties, the thermal conductivity in the fibre direction, $k_{1}$, is assumed to be constant according to the experiment observation $[27,82]$.

The variation of transverse thermal conductivity, $k_{2}$, is shown in Fig. 2 (a). The temperature ranging from $348 \mathrm{~K}$ to $373 \mathrm{~K}$ (shown in blue) represents the glass transition region, as $T_{g_{-} \text {low }}=348 \mathrm{~K}$ to represent the lower glass transition temperature and $T_{g_{-} \text {high }}=373 \mathrm{~K}$ to represent the higher glass transition temperature. It can be observed that the transverse thermal conductivity decreases with temperature increasing in glass transition region. When the temperature is higher than $573 \mathrm{~K}\left(T_{\text {ignited }}=573 \mathrm{~K}\right)$, the composite is ignited. Then the material property is assumed to be constant for $T>573 \mathrm{~K}$ due to the lack of measured data. The variation of $k_{2}$ is represented as

$$
k_{2}(\mathrm{~W} /(\mathrm{m} \cdot \mathrm{K}))= \begin{cases}0.362+7.57 \times 10^{-4} T, & 273 \mathrm{~K}<T \leq 348 \mathrm{~K} \\ 1.488-2.48 \times 10^{-3} T, & 348 \mathrm{~K}<T \leq 373 \mathrm{~K} \\ 0.104+1.23 \times 10^{-3} T, & 373 \mathrm{~K}<T \leq 573 \mathrm{~K} \\ 0.809, & 573 \mathrm{~K}<T\end{cases}
$$

The variation of specific heat capacity, $c_{v}$, is shown in Fig. 2 (b) and represented as

$$
c_{v}(\mathrm{~J} /(\mathrm{Kg} \cdot \mathrm{K}))= \begin{cases}-204+3.692 T, & 273 \mathrm{~K}<T \leq 348 \mathrm{~K} \\ -2775+11.08 T, & 348 \mathrm{~K}<T \leq 373 \mathrm{~K} \\ 665+1.858 T, & 373 \mathrm{~K}<T \leq 573 \mathrm{~K} \\ 1730, & 573 \mathrm{~K}<T\end{cases}
$$




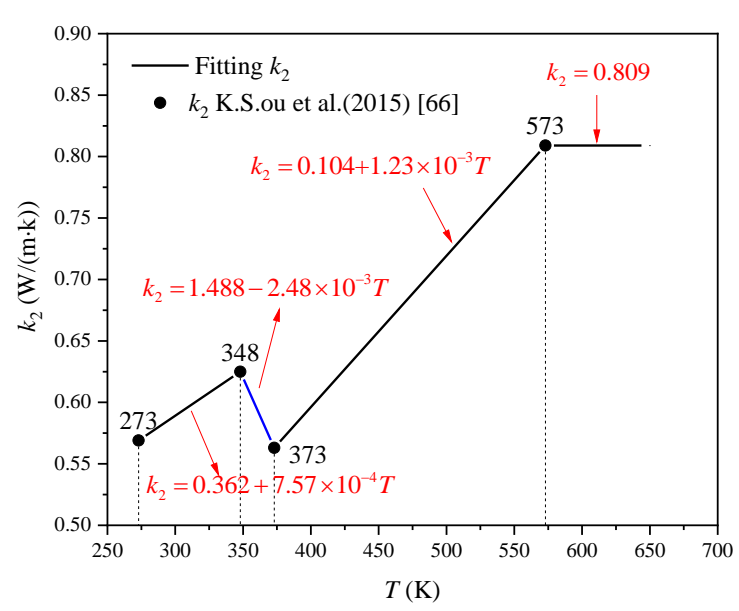

(a)

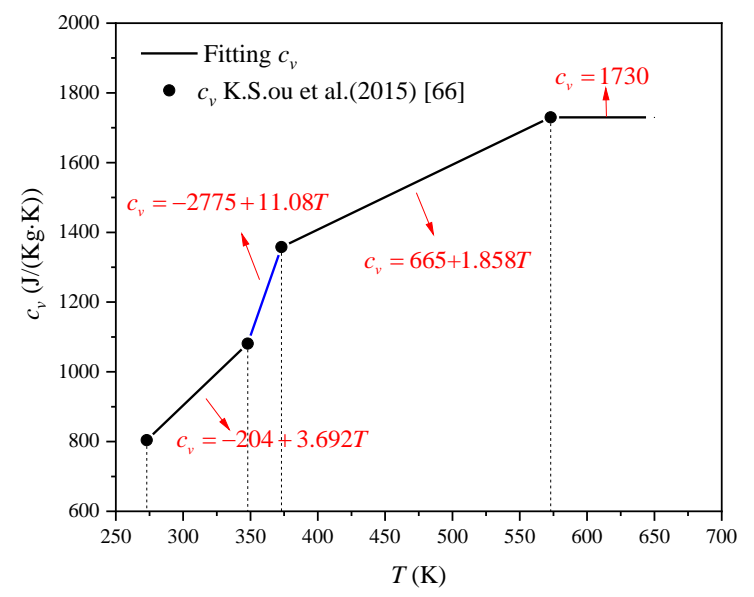

(b)

Fig. 2 Temperature dependent (a) transverse thermal conductivity $k_{2}$ and (b) specific heat capacity $c_{v}$

To represent the mechanical material properties, the following function is used [33]

$$
C(T)=\frac{C_{0}+C_{\infty}}{2}-\frac{C_{0}-C_{\infty}}{2} \tanh \left(C_{1}\left(T-T_{g_{-} h i g h}\right)\right)
$$

where $C$ represents the material property, $C_{0}$ represents the unrelaxed (initial) material property, and $C_{\infty}$ the represents the relaxed (final) property. The term $C_{1}$ is a constant value and $T_{g_{-} h i g h}$ represents the higher glass transition temperature as $T_{g_{-} h i g h}=373 \mathrm{~K}$ in the current composite material. By using the Matlab curve fitting tool, the data is presented in Fig. 3:

The elastic modulus in the transverse direction, $E_{2}$, is presented as

$$
E_{2}(\mathrm{GPa})=\left\{\begin{array}{lc}
4.645-4.529 \tanh (0.02177(T-373)) & , 273 \mathrm{~K} \leq T<475 \mathrm{~K} \\
0.1, & 475 \mathrm{~K} \leq T
\end{array}\right.
$$

The major shear modulus, $G_{12}$, is represented as

$$
G_{12}(\mathrm{GPa})=2.29-2.238 \tanh (0.0199(T-373))
$$




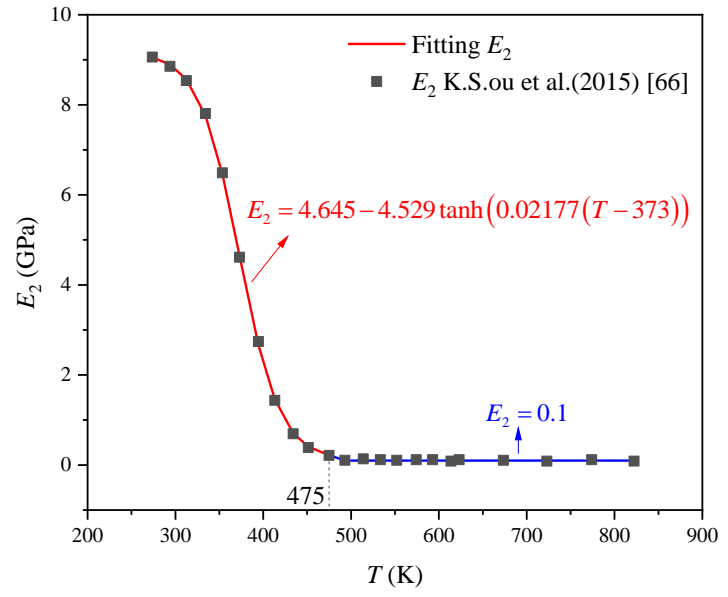

(a)

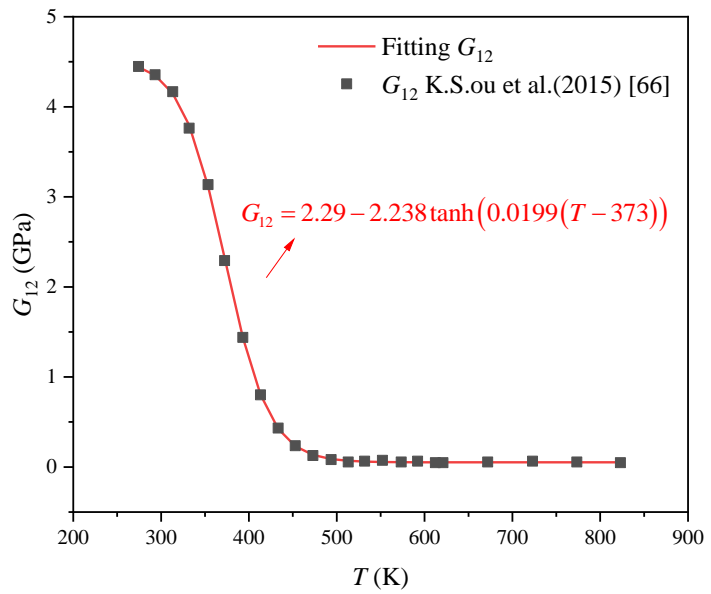

(b)

Fig. 3 Temperature dependent (a) transverse elastic modulus $E_{2}$ and (b) major shear modulus $G_{12}$

Instead of using Eq.(4) for fitting the elastic modulus in the fibre direction, $E_{1}$ is fitted as Eq.(7) which can give a better fitting result as

$$
E_{1}(\mathrm{GPa})=\left\{\begin{array}{l}
146.64937-0.06535 T, 273 \mathrm{~K} \leq T<475 \mathrm{~K} \\
110.86495+905.51983 e^{(-T / 91.34695)}, 475 \mathrm{~K} \leq T<825 \mathrm{~K}
\end{array}\right.
$$

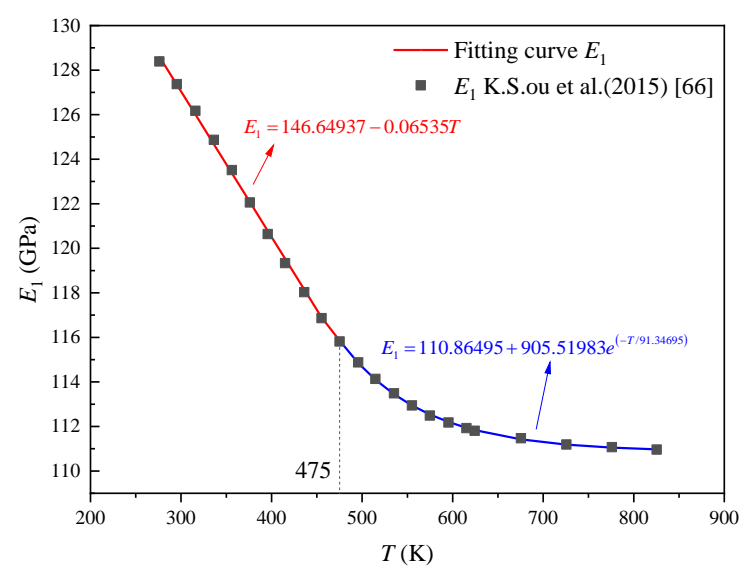

Fig. 4 Temperature dependent elastic modulus in fire direction 


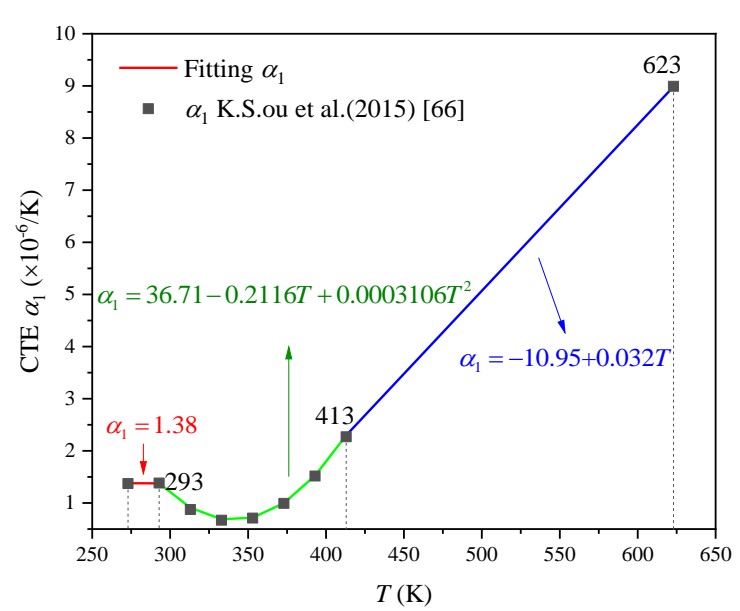

(a)

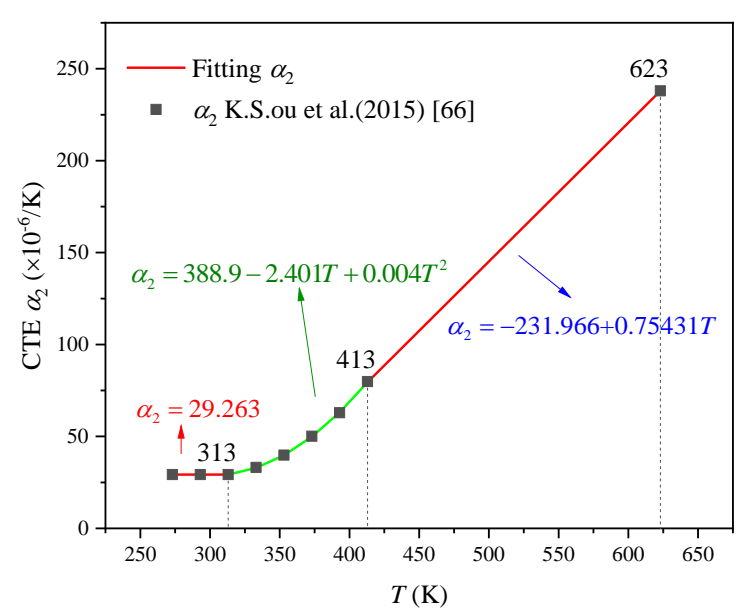

(b)

Fig. 5 Temperature dependent (a) CTE in fibre direction $\alpha_{1}$, (b) CTE in transverse direction $\alpha_{2}$

The coefficients of the thermal expansion (CTE) both in fibre direction $\alpha_{1}$ and in transverse direction $\alpha_{2}$ are piecewise fitted as Fig. 5 and presented as

$$
\begin{aligned}
& \alpha_{1}\left(\times 10^{-6} / \mathrm{K}\right)=\left\{\begin{array}{lr}
1.38, & 273 \mathrm{~K}<T \leq 293 \mathrm{~K} \\
36.71-0.2116 T+0.0003106 T^{2}, & 293 \mathrm{~K}<T \leq 413 \mathrm{~K} \\
-10.95+0.032 T, & 413 \mathrm{~K}<T \leq 623 \mathrm{~K}
\end{array}\right. \\
& \alpha_{2}\left(\times 10^{-6} / \mathrm{K}\right)=\left\{\begin{array}{lr}
29.263, & 273 \mathrm{~K}<T \leq 313 \mathrm{~K} \\
388.9-2.401 T+0.004 T^{2}, & 313 \mathrm{~K}<T \leq 413 \mathrm{~K} \\
-231.966+0.75431 T, & 413 \mathrm{~K}<T \leq 623 \mathrm{~K}
\end{array}\right.
\end{aligned}
$$

According to the PD failure criterion, the critical energy release rate is an important property in the damage simulation. The variation of energy release rate [83] is adopted as

$$
G_{I C}\left(\mathrm{~J} / \mathrm{m}^{2}\right)=\left\{\begin{array}{lc}
265.568+3.49 T-0.00745 T^{2}, & 273 \mathrm{~K} \leq T<535 \mathrm{~K} \\
0, & 535 \mathrm{~K} \leq T
\end{array}\right.
$$




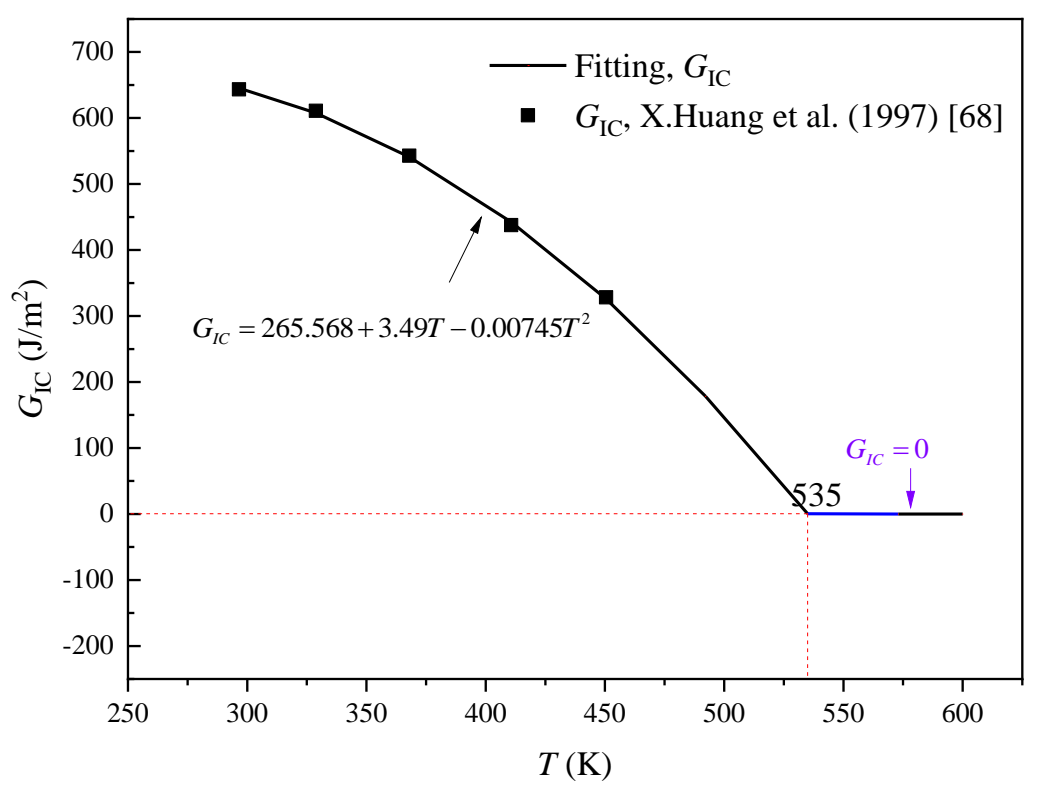

Fig. 6 Temperature dependent critical energy release rate $G_{I C}$

It is assumed that the bulk modulus $K_{m}(T)$ and Lamé constant $\mu_{m}(T)$ of the matrix material are linked to the transverse elastic modulus as [47]

$$
\begin{aligned}
& K_{m}(T)=\frac{E_{2}(T)}{2\left(1-v_{12}\right)} \\
& \mu_{m}(T)=\frac{E_{2}(T)}{2\left(1+v_{12}\right)}
\end{aligned}
$$

with the major Poisson's ratio being assumed to be a constant as $v_{12}=0.33$.

\subsection{PD mechanical model for composite material}

Peridynamic theory (PD) [34] belongs to the non-local theory, which uses integral equations instead of differential equations. The PD point can interact with other points within a distance called horizon size. Therefore, peridynamics provides an alternative way for solid and fluid mechanics problems. In this section, the PD composite model including temperature degradation is introduced [63]. Because of the temperature dependent material properties in classical continuum mechanics, the corresponding PD parameters also become temperature dependent, i.e. they are functions of temperature. Consequently, the formulations of the PD composite model are expressed as follows. 


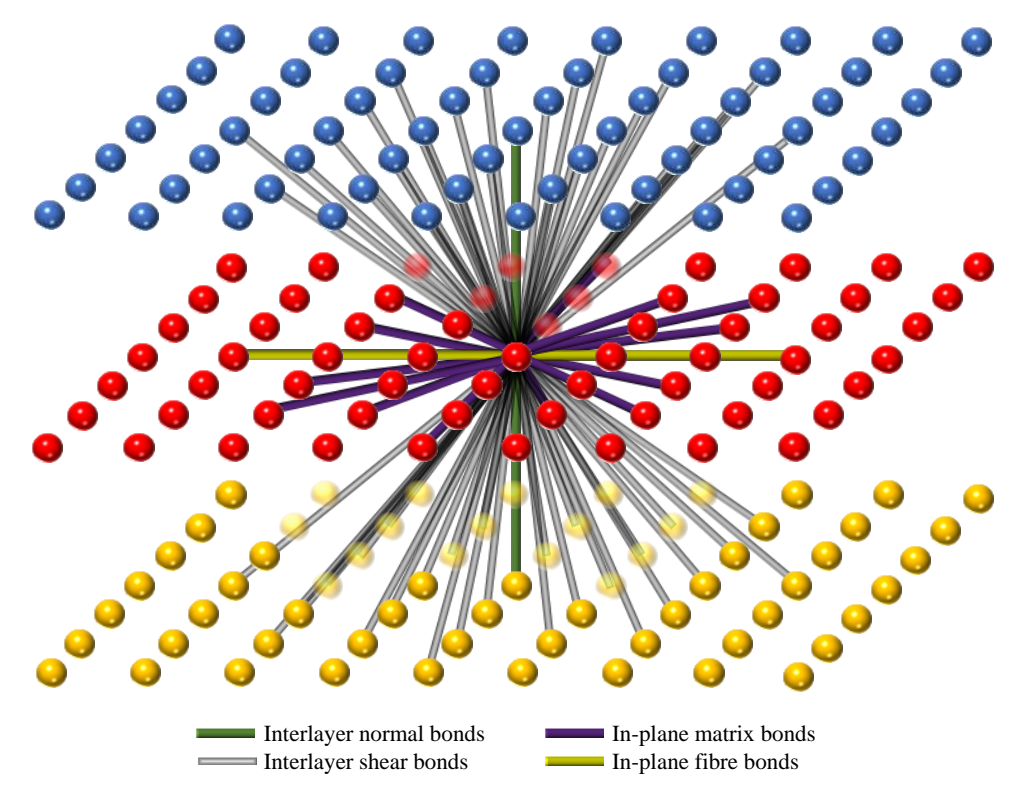

Fig. 7 Four types of PD bonds in a PD laminate mechanical model, for horizon size $\delta=3 \Delta x$ and fibre direction $\Phi=0$

Due to the directional dependency of composite material properties, there are four types of PD bonds existing in the laminate PD mechanical model [47], i.e. in-plane matrix bonds, inplane fibre bonds, interlayer normal bonds, and interlayer shear bonds, as illustrated in Fig. 7. The ordinary state-based formulation of PD equation of motion for composite material is described as [47]

$$
\rho_{s} \ddot{\mathbf{u}}_{s}\left(\mathbf{x}_{s, i}^{n}, t\right)=\sum_{j=1}^{N_{s, p l y, i}}\left(\mathbf{t}_{s, i j}^{n}-\mathbf{t}_{s, j i}^{n}\right) V_{s, j}^{n}+\sum_{m=n+1, n-1} \mathbf{P}_{s, i}^{n m} V_{s, i}^{m}+2 \sum_{m=n+1, n-1} \sum_{j=1}^{N_{s, s h e a r, i}} \mathbf{q}_{s, i j}^{n m} V_{s, j}^{m}+\mathbf{b}_{s}\left(\mathbf{x}_{s, i}^{n}, t\right)
$$

where $\rho_{s}$ is the density of composite. The point of interested is denoted by its initial coordinate as $\mathbf{x}_{s, i}^{n}$, where the subscript $\left({ }_{s}\right)$ denotes the composite and $\left({ }_{i}\right)$ denotes the point number. The superscript $\left({ }^{n}\right)$ represents the layer number. $\ddot{\mathbf{u}}_{s}$ is the acceleration and $\mathbf{b}_{s}$ is the volumetric body force. $N_{s, p l y, i}$ represents the total number of family members which are in the same ply of the interested point $\mathbf{x}_{s, i}^{n}$. On the other hand, $N_{s, s h e a r, i}$ represents the total number of the family members which interact with $\mathbf{x}_{s, i}^{n}$ through the interlayer shear bonds. $V_{s, j}^{n}$ represents the volume of material point at $\mathbf{x}_{s, j}^{n}$. The second term on the right-hand side of Eq.(13) represents the PD forces associated with interlayer normal bonds [47], as shown in Fig. 8 in blue. Here $m$ represents the number of the adjacent layer of $n^{\text {th }}$ ply, i.e. $m=n+1$ or $m=n-1$. The interlayer normal PD force density between point $\mathbf{x}_{s, i}^{n}$ and $\mathbf{x}_{s, i}^{m}$ is denoted by $\mathbf{P}_{s, i}^{n m}$. The third 
term on the right-hand side of Eq.(13) represents the PD forces associated with interlayer shear bonds [47], as shown in Fig. 8 in black. The term $\mathbf{q}_{s, i j}^{n m}$ represents the PD interlayer shear force density between points $\mathbf{x}_{s, i}^{n}$ and $\mathbf{x}_{s, j}^{m}$.

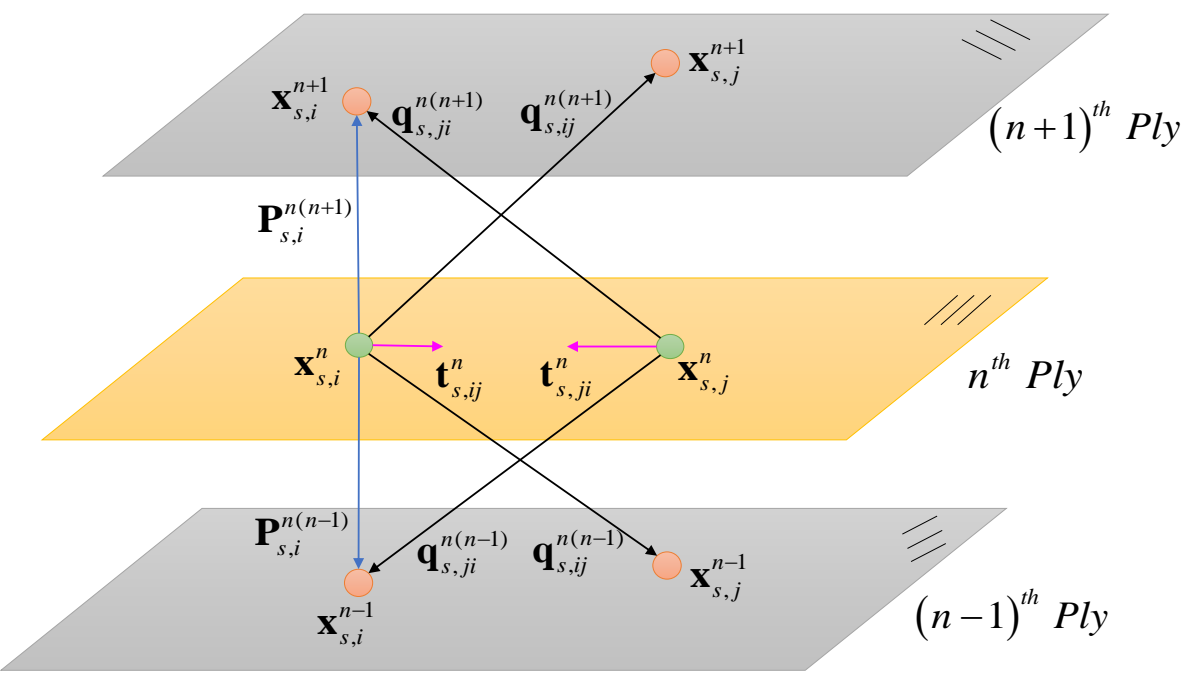

Fig. 8 Illustration of PD force densities for the point of interested $\mathbf{x}_{s, i}^{n}$

The first term on the right-hand side of Eq.(13) represents the PD forces associated with inplane bonds, including the in-plane matrix bonds and in-plane fibre bonds, as illustrated in Fig. 8 (shown in pink). The terms $\mathbf{t}_{s, i j}^{n}$ and $\mathbf{t}_{s, j i}^{n}$ are the PD force densities between points $\mathbf{x}_{s, i}^{n}$ and $\mathbf{x}_{s, j}^{n}[47]$ as

$\mathbf{t}_{s, i j}^{n}=2 \delta\left[\begin{array}{l}\frac{d \Lambda_{i j}}{\left|\mathbf{x}_{s, j}^{n}-\mathbf{x}_{s, i}^{n}\right|} a_{s, i}\left(\theta_{s, i}-\left(\alpha_{1, i}+\alpha_{2, i}\right)\left(T_{s, i}^{n}-T_{s, i}^{n, 0}\right)\right) \\ +\left(\mu_{f} \chi_{F} b_{F, i}+\mu_{m} \chi_{T} b_{T, i}+\mu_{m} b_{F T, i}\right)\left(s_{i j}-\alpha_{\varphi, i j}\left(T_{s, i}^{n}-T_{s, i}^{n, 0}\right)\right)\end{array}\right] \frac{\mathbf{y}_{s, j}^{n}-\mathbf{y}_{s, i}^{n}}{\left|\mathbf{y}_{s, j}^{n}-\mathbf{y}_{s, i}^{n}\right|}$

and

$$
\begin{aligned}
& \mathbf{t}_{s, j i}^{n}= \\
& -2 \delta\left[\begin{array}{l}
\frac{d \Lambda_{i j}}{\left|\mathbf{x}_{s, j}^{n}-\mathbf{x}_{s, i}^{n}\right|} a_{s, j}\left(\theta_{s, j}-\left(\alpha_{1, j}+\alpha_{2, j}\right)\left(T_{s, j}^{n}-T_{s, j}^{n, 0}\right)\right) \\
+\left(\mu_{f} \chi_{F} b_{F, j}+\mu_{m} \chi_{T} b_{T, j}+\mu_{m} b_{F T, j}\right)\left(s_{i j}-\alpha_{\varphi, i j}\left(T_{s, j}^{n}-T_{s, j}^{n, 0}\right)\right)
\end{array}\right] \mid \frac{\mathbf{y}_{s, j}^{n}-\mathbf{y}_{s, i}^{n}}{\left|\mathbf{y}_{s, j}^{n}-\mathbf{y}_{s, i}^{n}\right|}
\end{aligned}
$$


where $T_{s, i}^{n, 0}$ and $T_{s, j}^{n, 0}$ represent the initial temperature of point $\mathbf{x}_{s, i}^{n}$ and $\mathbf{x}_{s, j}^{n}$. The term $\mathbf{y}_{s, i}^{n}$ represents the updated position of material point at $\mathbf{x}_{s, i}^{n}$. The horizon size for the in-plane PD interactions is denoted by $\delta$.

The PD stretch $s_{i j}$ and the auxiliary parameters $\Lambda_{i j}$ and $d$ are [47]

$$
\begin{gathered}
s_{i j}=\frac{\left|\mathbf{y}_{s, j}^{n}-\mathbf{y}_{s, i}^{n}\right|-\left|\mathbf{x}_{s, j}^{n}-\mathbf{x}_{s, i}^{n}\right|}{\left|\mathbf{x}_{s, j}^{n}-\mathbf{x}_{s, i}^{n}\right|} \\
\Lambda_{k j}=\left(\frac{\mathbf{y}_{s, j}^{n}-\mathbf{y}_{s, i}^{n}}{\left|\mathbf{y}_{s, j}^{n}-\mathbf{y}_{s, i}^{n}\right|}\right) \cdot\left(\frac{\mathbf{x}_{s, j}^{n}-\mathbf{x}_{s, i}^{n}}{\left|\mathbf{x}_{s, j}^{n}-\mathbf{x}_{s, i}^{n}\right|}\right) \\
d=\frac{2}{\pi h_{\text {thick }} \delta^{3}}
\end{gathered}
$$

where $h_{\text {thick }}$ represents the thickness of one ply.

The supplementary parameters $\chi_{F}$ and $\chi_{T}$ are defined as

$$
\chi_{F}=\left\{\begin{array}{l}
1,\left(\mathbf{x}_{s, j}^{n}-\mathbf{x}_{s, i}^{n}\right) / / \text { fibre direction } \\
0, \text { otherwise }
\end{array}\right.
$$

and

$$
\chi_{T}=\left\{\begin{array}{l}
1,\left(\mathbf{x}_{s, j}^{n}-\mathbf{x}_{s, i}^{n}\right) \perp \text { fibre direction } \\
0, \text { otherwise }
\end{array}\right.
$$

The PD parameters in Eq.(14) and (15) [47] can be described as functions of temperature as

$$
\begin{gathered}
a_{s, i}=\frac{1}{2}\left(Q_{12 . i}-Q_{66, i}\right) \\
b_{F T, i}=\frac{6 Q_{66, i}}{\pi h_{\text {thick }} \delta^{4}} \\
b_{F, i}=\frac{\left(Q_{11, i}-Q_{12, i}-2 Q_{66, i}\right)}{2 \delta\left(\sum_{j=1}^{N_{s, f i b r e, i}}\left|\mathbf{x}_{s, j}^{n}-\mathbf{x}_{s, i}^{n}\right| V_{s, j}^{n}\right)}
\end{gathered}
$$




$$
b_{T, i}=\frac{\left(Q_{22, i}-Q_{12, i}-2 Q_{66, i}\right)}{2 \delta\left(\sum_{j=1}^{N_{s, f i b r e, i}}\left|\mathbf{x}_{s, j}^{n}-\mathbf{x}_{s, i}^{n}\right| V_{s, j}^{n}\right)}
$$

where $N_{s, f i b r e, i}$ represents the number of the family members of point $\mathbf{x}_{s, i}^{n}$ interacted through in-plane fibre bonds. The reduced stiff matrix parameters [84] are considered as

$$
Q_{11, i}=\frac{E_{1, i}}{1-v_{12} v_{21}}, Q_{22, i}=\frac{E_{2, i}}{1-v_{12} v_{21}}, Q_{12, i}=\frac{v_{12} E_{2, i}}{1-v_{12} v_{21}}, Q_{66, i}=G_{12, i}, v_{21}=v_{12} E_{2} / E_{1}
$$

The material parameters $E_{2, i}, G_{12, i}, E_{1, i}, \alpha_{1, i}, \alpha_{2, i}$ at position $\mathbf{x}_{s, i}^{n}$ are calculated as functions of temperature $T_{s, i}^{n}$ as provided in Eqs. (5)-(9). On the other hand, the minor Poisson's ratio, $v_{21}$, is calculated at the initial temperature $T_{s}^{0}$. The coefficient of the thermal expansion for an arbitrary direction $\alpha_{\varphi, i j}[48]$ is defined as

$$
\alpha_{\varphi, i j}=\alpha_{x, i} \cos ^{2}(\varphi)+\alpha_{y, i} \sin ^{2}(\varphi)+\alpha_{x y, i} \sin (\varphi) \cos (\varphi)
$$

where

$$
\begin{gathered}
\alpha_{x, i}=\alpha_{1, i} \cos ^{2}(\Phi)+\alpha_{2, i} \sin ^{2}(\Phi) \\
\alpha_{y, i}=\alpha_{1, i} \sin ^{2}(\Phi)+\alpha_{2, i} \cos ^{2}(\Phi) \\
\varphi=\arcsin \left(\frac{\left(\mathbf{x}_{s, j}^{n}-\mathbf{x}_{s, i}^{n}\right) \cdot \mathbf{e}_{x}}{\left|\mathbf{x}_{s, j}^{n}-\mathbf{x}_{s, i}^{n}\right|}\right)
\end{gathered}
$$

where $\varphi$ represents the PD bond angle with positive $x$ direction and $\mathbf{e}_{x}$ represents the unit vector in the positive $x$ direction. The term $\Phi$ represents the fibre direction.

The parameters $\theta_{s, i}$ and $\theta_{s, j}$ in Eqs.(14) and (15) are dilatation terms [47, 54] which are defined by using the temperature dependent parameters as

$$
\theta_{s, i}\left(\mathbf{x}_{s, i}^{n}, t\right)=d \delta \sum_{j=1}^{N_{s, p l y, i}} \mu_{d}\left(s_{i j}-\alpha_{\varphi, i j}\left(T_{s, i}^{n}-T_{s, i}^{n, 0}\right)\right) \Lambda_{i j} V_{s, j}^{n}+\left(\alpha_{1, i}+\alpha_{2, i}\right)\left(T_{s, i}^{n}-T_{s, i}^{n, 0}\right)
$$

and 


$$
\theta_{s, j}\left(\mathbf{x}_{s, j}^{n}, t\right)=d \delta \sum_{i=1}^{N_{s, n l y, j}} \mu_{d}\left(s_{i j}-\alpha_{\varphi, i j}\left(T_{s, j}^{n}-T_{s, j}^{n, 0}\right)\right) \Lambda_{i j} V_{s, i}^{n}+\left(\alpha_{1, j}+\alpha_{2, j}\right)\left(T_{s, j}^{n}-T_{s, j}^{n, 0}\right)
$$

with

$$
\mu_{d}=\left\{\begin{array}{l}
\mu_{f}, \text { for }\left(\mathbf{x}_{s, j}^{n}-\mathbf{x}_{s, i}^{n}\right) / / \text { fibre direction } \\
\mu_{m}, \text { otherwise }
\end{array}\right.
$$

The PD parameters accounting for the interlayer interactions are assumed to be temperature independent. Consequently, the PD force densities associated with interlayer bonds are defined as $[47,54]$

$$
\mathbf{P}_{s, i}^{n m}=\mu_{i n} 4 b_{N} \hat{\delta}\left(s_{i}^{n m}-\alpha_{m}\left(T_{s \text { avg }, i}^{n m}-T_{s a v g, i}^{n m, 0}\right)\right) \frac{\mathbf{y}_{s, i}^{m}-\mathbf{y}_{s, i}^{n}}{\left|\mathbf{y}_{s, i}^{m}-\mathbf{y}_{s, i}^{n}\right|}
$$

and

$$
\mathbf{q}_{s, i j}^{n m}=\mu_{i s} 4 b_{s} \tilde{\delta}\left(\left(s_{i j}^{n m}-\alpha_{m}\left(T_{s a v g, i j}^{n m}-T_{s a v g, i j}^{n m, 0}\right)\right)-\left(s_{j i}^{n m}-\alpha_{m}\left(T_{s a v g, j i}^{n m}-T_{s a v g, j i}^{n m, 0}\right)\right)\right) \frac{\mathbf{y}_{s, j}^{m}-\mathbf{y}_{s, i}^{n}}{\left|\mathbf{y}_{s, j}^{m}-\mathbf{y}_{s, i}^{n}\right|}
$$

with

$$
\begin{gathered}
T_{s \text { avg }, i j}^{n m}=\left(T_{s, i}^{n}+T_{s, j}^{m}\right) / 2, T_{s \text { avg }, j i}^{n m}=\left(T_{s, i}^{m}+T_{s, j}^{n}\right) / 2, T_{s \text { avg }, i}^{n m}=\left(T_{s, i}^{n}+T_{s, i}^{m}\right) / 2 \\
T_{s \text { avg }, i j}^{n m, 0}=\left(T_{s, i}^{n, 0}+T_{s, j}^{m, 0}\right) / 2, T_{s \text { avg }, j i}^{n m, 0}=\left(T_{s, i}^{m, 0}+T_{s, j}^{n, 0}\right) / 2, T_{s \text { avg }, i}^{n m, 0}=\left(T_{s, i}^{n, 0}+T_{s, i}^{m, 0}\right) / 2 \\
s_{i j}^{n m}=\frac{\left|\mathbf{y}_{s, j}^{m}-\mathbf{y}_{s, i}^{n}\right|-\left|\mathbf{x}_{s, j}^{m}-\mathbf{x}_{s, i}^{n}\right|}{\left|\mathbf{x}_{s, j}^{m}-\mathbf{x}_{s, i}^{n}\right|}, s_{j i}^{n m}=\frac{\left|\mathbf{y}_{s, j}^{n}-\mathbf{y}_{s, i}^{m}\right|-\left|\mathbf{x}_{s, j}^{n}-\mathbf{x}_{s, i}^{m}\right|}{\left|\mathbf{x}_{s, j}^{n}-\mathbf{x}_{s, i}^{m}\right|}, \\
s_{i}^{n m}=\frac{\left|\mathbf{y}_{s, i}^{n}-\mathbf{y}_{s, i}^{m}\right|-\left|\mathbf{x}_{s, i}^{n}-\mathbf{x}_{s, i}^{m}\right|}{\left|\mathbf{x}_{s, i}^{n}-\mathbf{x}_{s, i}^{m}\right|}
\end{gathered}
$$

where $\mathbf{y}_{s, i}^{m}$ and $\mathbf{y}_{s, j}^{m}$ represent the updated positions of points $\mathbf{x}_{s, i}^{m}$ and $\mathbf{x}_{s, j}^{m}$ located in the $m^{\text {th }}$ ply, as illustrated in Fig. 8. The term $\alpha_{m}$ is the CTE of matrix material as $\alpha_{m}=63 \times 10^{-6} / \mathrm{K}$ [54].

The definitions of these interlayer PD parameters are defined as [47] 


$$
\begin{aligned}
& b_{N}=\frac{E_{m}}{2 h_{\text {thick }} \hat{\delta}\left[V_{s, i}^{n+1}+V_{s, i}^{n-1}\right]} ; \\
& b_{S}=\frac{G_{m}}{16 \pi \tilde{\delta} h_{\text {thick }}^{3}\left(\left(\delta^{2}+2 h_{\text {thick }}^{2}\right) / \tilde{\delta}-2 h_{\text {thick }}\right)}
\end{aligned}
$$

where $E_{m}$ and $G_{m}$ are the elastic and shear moduli of the matrix material. $V_{s, i}^{n+1}$ and $V_{s, i}^{n-1}$ are the volumes of points at $\mathbf{x}_{s, i}^{n+1}$ and $\mathbf{x}_{s, i}^{n-1}$. In the above equations, $\hat{\delta}$ and $\tilde{\delta}$ represent the horizon size in the interlayer normal direction and interlayer shear direction as $\hat{\delta}=h_{\text {thick }}$ and $\tilde{\delta}=\sqrt{h_{\text {thick }}^{2}+\delta^{2}}[47]$.

In Eq.(14) and (15), the parameters $\mu_{f}, \mu_{m}$ are the failure parameters in the fibre and all other directions for lamina. On the other hand, in Eq.(33) and (34), the parameters $\mu_{i n}$ and $\mu_{i s}$ are the failure parameters for interlayer normal bonds and interlayer shear bonds [47]. These failure parameters are utilized to indicate the breakage of PD bonds. If the stretch of a bond is larger than the critical stretch, the bond is broken by considering the thermal degradation properties of the composites for different kinds of PD mechanical bonds as

$$
\begin{aligned}
& \mu_{f}\left(\mathbf{x}_{s, i}^{n}, \mathbf{x}_{s, j}^{n}, t\right)=\left\{\begin{array}{l}
1,\left(s_{i j}-\alpha_{1, i}\left(T_{\text {savg }, i j}^{n}-T_{s a v g, i j}^{n, 0}\right)\right)<s_{c f, i} \\
0,\left(s_{i j}-\alpha_{1, i}\left(T_{s a v g, i j}^{n}-T_{s a v g, i j}^{n, 0}\right)\right) \geq s_{c f, i}
\end{array}\right. \\
& \mu_{m}\left(\mathbf{x}_{s, i}^{n}, \mathbf{x}_{s, j}^{n}, t\right)=\left\{\begin{array}{l}
1,\left(s_{i j}-\alpha_{\varphi, i j}\left(T_{s a v g, i j}^{n}-T_{s a v g, i j}^{n, 0}\right)\right)<s_{c m, i} \\
0,\left(s_{i j}-\alpha_{\varphi, i j}\left(T_{s a v g, i j}^{n}-T_{s a v g, i j}^{n, 0}\right)\right) \geq s_{c m, i}
\end{array}\right. \\
& \mu_{i n}\left(\mathbf{x}_{s, i}^{n}, \mathbf{x}_{s, i}^{m}, t\right)=\left\{\begin{array}{l}
1,\left(s_{i}^{n m}-\alpha_{m}\left(T_{\text {savg }, i}^{n m}-T_{s \text { avg }, i}^{n m, 0}\right)\right)<s_{\text {cin }, i} \\
0,\left(s_{i}^{n m}-\alpha_{m}\left(T_{s \text { avg }, i}^{n m}-T_{s \text { avg }, i}^{n m, 0}\right)\right) \geq s_{\text {cin }, i}
\end{array}\right. \\
& \mu_{i s}\left(\mathbf{x}_{s, i}^{n}, \mathbf{x}_{s, j}^{m}, t\right)=\left\{\begin{array}{l}
1,\left(\begin{array}{l}
\left(s_{i j}^{n m}-\alpha_{m}\left(T_{s \text { avg }, i j}^{n m}-T_{s \text { avg }, i j}^{n m, 0}\right)\right) \\
-\left(s_{j i}^{n m}-\alpha_{m}\left(T_{s a v g, j i}^{n m}-T_{s \text { avg }, j i}^{n m, 0}\right)\right)
\end{array}\right)<s_{c i s, i j} \\
0,\left(\begin{array}{l}
\left(s_{i j}^{n m}-\alpha_{m}\left(T_{s \text { avg }, i j}^{n m}-T_{s \text { avg }, i j}^{n m, 0}\right)\right) \\
-\left(s_{j i}^{n m}-\alpha_{m}\left(T_{s a v g, j i}^{n m}-T_{s \text { avg }, j i}^{n m, 0}\right)\right)
\end{array}\right) \geq s_{c i s, i j}
\end{array}\right.
\end{aligned}
$$


where $s_{c f, i}, s_{c m, i}$ are the critical stretches in the fibre and all other directions for lamina. On the other hand, $s_{c i n, i}$, and $s_{c i s, i j}$ are the critical stretches for interlayer normal and interlayer shear bonds. Note that the critical stretch values are calculated at temperature value $T_{s, i}^{n}$. The critical stretches can be calculated as $[36,47]$

$$
\begin{gathered}
s_{c f, i}=\frac{\sigma_{1 t}}{E_{1, i}} \\
s_{c m, i}=\sqrt{\frac{G_{I C, i}}{\left(\frac{6}{\pi} \mu_{m, i}+\frac{16}{9 \pi^{2}}\left(K_{m, i}-2 \mu_{m, i}\right)\right) \delta}} \\
s_{c i s, i j}=\frac{2}{\mid \mathbf{x}_{s, i}^{n}-\mathbf{x}_{s, j}^{m}} \mid \sqrt{\frac{2 G_{I C, i}}{h_{t h i c k} E_{m}}}
\end{gathered}
$$

where $\sigma_{1 t}$ is the tensile strength in the fibre direction. In the present study, the value of $\sigma_{1 t}=2550 \mathrm{MPa}$ is chosen [85]. The term $G_{I I C}$ is the critical strain energy release rate for the second failure mode. In this study, the critical strain energy release rate for the second failure mode $G_{I I C}$ is simply assumed to be equal to the critical strain energy release rate for the first failure mode, $G_{I C} \cdot E_{m}$ and $G_{m}$ are the elastic and shear moduli of the matrix material, which have the values as $E_{m}=3.4 \mathrm{GPa}$ and $G_{m}=1.308 \mathrm{GPa}$ [49]. The material parameters $E_{1, i}, \mu_{m, i}$, $K_{m, i}$, and $G_{I C, i}$ are calculated at temperature $T_{s, i}^{n}$ as provided in section 2.2.

The variation of the critical stretch, $s_{c m, i}$ as a function of temperature is plotted in Fig. 9. It can be observed from the figure that within the range of $273 \mathrm{~K}<T \leq 348 \mathrm{~K}$, the material remains as a brittle material before the glass transition $[1,86]$. Then the composite material becomes a rubber-like ductile material $[1,86]$ for the approximate region $348 \mathrm{~K}<T \leq 493 \mathrm{~K}$. Then when the temperature is larger than the critical value of $493 \mathrm{~K}$, the critical stretch decreases as shown in Fig. 9. Finally, the material totally fails (critical stretch equals to zero) when the temperature is higher than $535 \mathrm{~K}[86]$. 


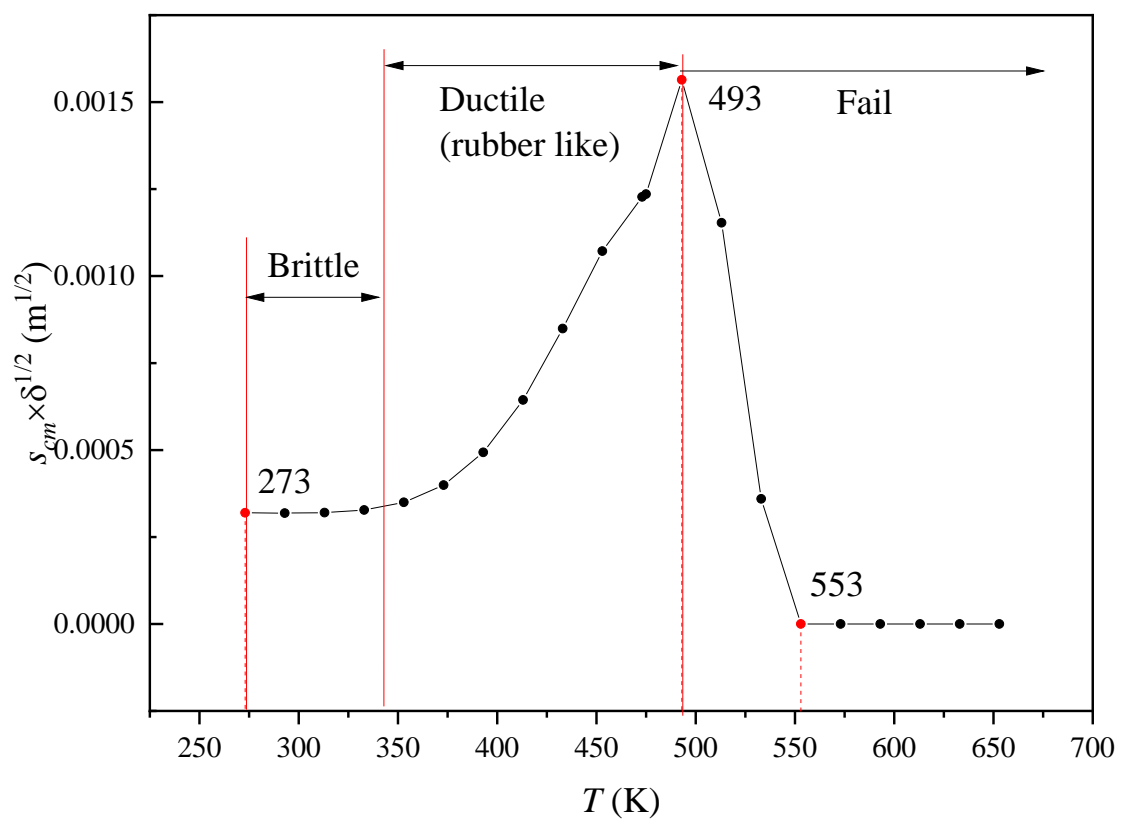

Fig. 9 The variation of $s_{c m} \delta^{1 / 2}$ as a function of temperature

The local damage parameters which represent the damage level are expressed as [63]

$$
\begin{aligned}
& \Psi_{f}\left(\mathbf{x}_{s, i}^{n}, t\right)=1-\frac{\sum_{j=1}^{N_{s, \text { fibre, } i}} \mu_{f}\left(\mathbf{x}_{s, i}^{n}, \mathbf{x}_{s, j}^{n}, t\right) V_{s, j}^{n}}{\sum_{j=1}^{N_{s, f i b r e, i}} V_{s, j}^{n}} \\
& \Psi_{m}\left(\mathbf{x}_{s, i}^{n}, t\right)=1-\frac{\sum_{j=1}^{N_{s, p l y, i}} \mu_{m}\left(\mathbf{x}_{s, i}^{n}, \mathbf{x}_{s, j}^{n}, t\right) V_{s, j}^{n}}{\sum_{j=1}^{N_{s, p l y, i}} V_{s, j}^{n}} \\
& \Psi_{i n}\left(\mathbf{x}_{s, i}^{n}, t\right)=1-\frac{\sum_{m=n+1, n-1} \mu_{i n}\left(\mathbf{x}_{s, i}^{n}, \mathbf{x}_{s, i}^{m}, t\right) V_{s, i}^{m}}{\sum_{m=n+1, n-1} V_{s, i}^{m}} \\
& \Psi_{i s}\left(\mathbf{x}_{s, i}^{n}, t\right)=1-\frac{\sum_{j=1}^{N_{s, s h e a r, i}} \mu_{i s}\left(\mathbf{x}_{s, i}^{n}, \mathbf{x}_{s, j}^{m}, t\right) V_{s, j}^{m}}{\sum_{j=1}^{N_{s, s h a a r, i}} V_{s, j}^{m}}
\end{aligned}
$$

It should be noted that the local damage parameters in Eqs.(47)-(50) are calculated by only considering mechanical deformation failure. 


\subsection{PD thermal model for composite material}

The heat conduction equation for multi-layer composite material in PD form can be expressed as $[54,55]$

$$
\begin{aligned}
& \rho_{s} c_{v, i}^{n} \frac{\partial T_{s, i}^{n}}{\partial t} \\
& =\sum_{j=1}^{N_{s, p l y, i}}\left[\left(\mu_{f} \chi_{F} \frac{\kappa_{F, i}^{n}+\kappa_{F, j}^{n}}{2}+\mu_{m} \frac{\kappa_{m, i}^{n}+\kappa_{m, j}^{n}}{2}\right) \frac{T_{s, j}^{n}-T_{s, i}^{n}}{\left|\mathbf{x}_{s, j}^{n}-\mathbf{x}_{s, i}^{n}\right|}\right] V_{s, j}^{n} \\
& +\sum_{m=n+1, n-1}\left[\mu_{i n} \kappa_{\mathrm{int} t r, i}^{n} \frac{T_{s, i}^{m}-T_{s, i}^{n}}{\left|\mathbf{x}_{s, i}^{m}-\mathbf{x}_{s, i}^{n}\right|}\right] V_{s, i}^{m}+\sum_{m=n+1, n-1} \sum_{j=1}^{N_{s, \text { shear }, i}}\left[\mu_{i s} \kappa_{\mathrm{int} e r, i}^{n} \frac{T_{s, j}^{m}-T_{s, i}^{n}}{\left|\mathbf{x}_{s, j}^{m}-\mathbf{x}_{s, i}^{n}\right|}\right] V_{s, j}^{m} \\
& +h_{s, i}^{n}
\end{aligned}
$$

where $c_{v, i}^{n}$ is the specific heat capacity as provided in Eq.(3), and $h_{s}$ is the volumetric heat source.

In Eq. (51), $\kappa_{F, i}^{n}$ represents the PD micro thermal conductivity in the fibre direction, $\kappa_{m, i}^{n}$ represents the PD micro thermal conductivity in all the other directions for lamina as [55]

$$
\begin{gathered}
\kappa_{F, i}^{n}=\frac{2\left(k_{1}-k_{2, i}\right)}{\sum_{j=1}^{N_{s, \text { fibre }, i}}\left|\mathbf{x}_{s, j}^{n}-\mathbf{x}_{s, i}^{n}\right| V_{s, j}^{n}} \\
\kappa_{m, i}^{n}=\frac{6 k_{2, i}}{\pi h_{\text {thick }} \delta^{3}}
\end{gathered}
$$

where $k_{2, i}$ is the thermal conductivity in the transverse direction located at position $\mathbf{x}_{s, i}^{n}$ and calculated at temperature $T_{s, i}^{n}$ as provided in Eq.(2). The parameter $k_{1}$ is the thermal conductivity in the fibre direction which is assumed constant as $k_{1}=8.3075 \mathrm{~W} /(\mathrm{m} \cdot \mathrm{K})[27,82]$.

In Eq. (51), $\kappa_{\text {inter, } i}^{n}$ represents the interlayer PD thermal conductivity as [54]

$$
\kappa_{\text {inter }, i}^{n}=\frac{k_{m}}{2 \pi h_{\text {thick }}^{3}\left(\tilde{\delta}-h_{\text {thick }}\right)}
$$


where $k_{m}$ is the thermal conductivity of matrix material which is assumed constant as $k_{m}=0.2 \mathrm{~W} /(\mathrm{m} \cdot \mathrm{K})[54]$. The parameters $c_{v, i}^{n}, h_{s, i}^{n}, \kappa_{F, i}^{n}, \kappa_{m, i}^{n}$, and $\kappa_{\text {inter }, i}^{n}$ are defined at location $\mathbf{x}_{s, i}^{n}$.

To represent the level of damage due to melting of composite material, a non-dimensional temperature, $\tilde{T}$, is defined as

$$
\tilde{T}=\frac{T_{g_{-} h i g h}-T_{s, i}^{n}}{T_{g_{-} h i g h}-T_{s, r e f}}
$$

and

$$
T_{s, i}^{n}=\left\{\begin{array}{c}
T_{\text {ignited }} \rightarrow \tilde{T}=-2.5 \\
T_{s, \text { ref }} \rightarrow \tilde{T}=1 \\
T_{g_{-} \text {high }} \rightarrow \tilde{T}=0 \\
T_{g_{-} \text {low }} \rightarrow \tilde{T}=0.3125
\end{array}\right.
$$

where $T_{s}$ is the temperature of the PD composite point, $T_{s, r e f}$ is the reference temperature $T_{s, \text { ref }}=293 \mathrm{~K}, T_{\text {ignited }}$ is the ignition temperature $T_{\text {ignited }}=573 \mathrm{~K}, T_{g_{-} \text {low }}$ is the lower glass transition temperature $T_{g_{-} \text {low }}=348 \mathrm{~K}$, and $T_{g_{-} \text {high }}$ is the higher glass transition temperature $T_{g_{-} \text {high }}=373 \mathrm{~K}[81]$. Therefore, $\tilde{T} \leq-2.5$ represents that the material is ignited, $\tilde{T} \leq 0$ represents the post glass transition period $\left(T_{s, i}^{n}>T_{g_{-} h i g h}\right)$, and $\tilde{T}=1$ represents composite is at the reference temperature, as illustrated in Fig. 10.

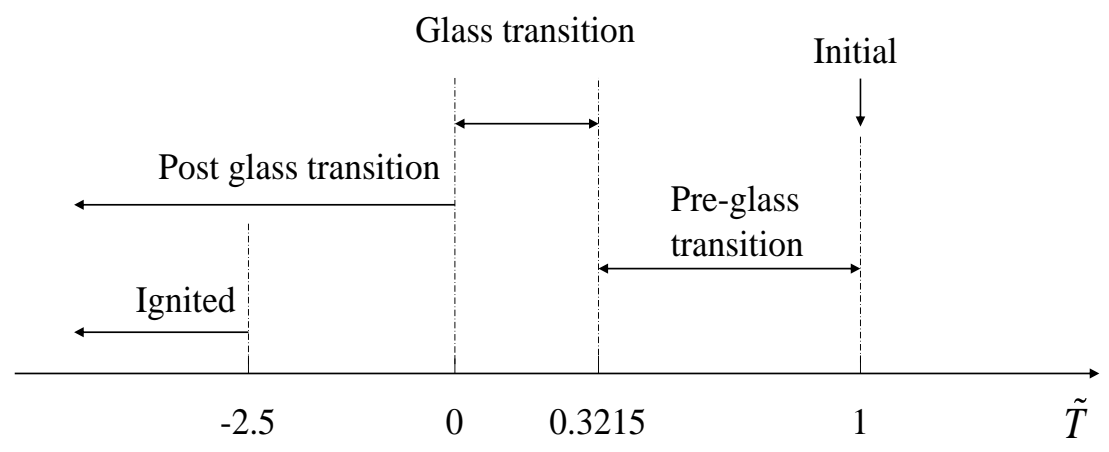

Fig. 10 Different domains distinguished by $\tilde{T} \quad$ [81] 


\section{Non-dimensional PD thermal model for composite and PD thermo-fluid model for hot gas flow}

\subsection{Non-dimensional PD thermo-fluid model}

In the current study, the PD thermo-fluid model [77] is used to represent the fire's thermofluid property. Furthermore, the fluid flow is assumed to be two-dimensional in the Eulerian description. In this study, the non-dimensional form of the non-local Naiver-Stokes equations [77] with Boussinesq approximation [87] is adopted by using the peridynamic differential operator (PDDO) [74].

The non-dimensional PDDO form of the governing equations are [77]:

Conservation of mass:

$$
\begin{aligned}
\frac{\partial \rho_{f, i}^{*}}{\partial t^{*}}=-\rho_{f, i}^{*} & \sum_{j=1}^{N_{f, i}}\left(\mathbf{v}_{f, j}^{*}-\mathbf{v}_{f, i}^{*}\right) \cdot \mathbf{g}_{1}\left(\mathbf{x}_{f, j}^{*}-\mathbf{x}_{f, i}^{*}\right) V_{f, j}^{*} \\
& -\sum_{j=1}^{N_{f, i}}\left(\rho_{f, j}^{*}-\rho_{f, i}^{*}\right)\left(\mathbf{v}_{f, i}^{*} \cdot \mathbf{g}_{1}\left(\mathbf{x}_{f, j}^{*}-\mathbf{x}_{f, i}^{*}\right)\right) V_{f, j}^{*}
\end{aligned}
$$

Conservation of momentum:

$$
\begin{aligned}
\frac{\partial \mathbf{v}_{f, i}^{*}}{\partial t^{*}}=-\sum_{j=1}^{N_{f, i}} & \left(P_{f, j}^{*}-P_{f, i}^{*}\right) \mathbf{g}_{1}\left(\mathbf{x}_{f, j}^{*}-\mathbf{x}_{f, i}^{*}\right) V_{f, j}^{*} \\
& +\operatorname{Pr} \mu_{f, i}^{*} \sum_{j=1}^{N_{f, i}}\left(\mathbf{v}_{f, j}^{*}-\mathbf{v}_{f, i}^{*}\right) \operatorname{Tr}\left(\mathbf{g}_{2}\left(\mathbf{x}_{f, j}^{*}-\mathbf{x}_{f, i}^{*}\right)\right) V_{f, j}^{*} \\
& -\sum_{j=1}^{N_{f, i}}\left(\mathbf{v}_{f, j}^{*}-\mathbf{v}_{f, i}^{*}\right)\left(\mathbf{v}_{f, i}^{*} \cdot \mathbf{g}_{1}\left(\mathbf{x}_{f, j}^{*}-\mathbf{x}_{f, i}^{*}\right)\right) V_{f, j}^{*} \\
& +\operatorname{Ra} \operatorname{Pr} T_{f, i}^{*}\left[\begin{array}{l}
0 \\
1
\end{array}\right]
\end{aligned}
$$

Conservation of energy:

$$
\begin{aligned}
\frac{\partial T_{f, i}^{*}}{\partial t^{*}}=k_{f, i}^{*} & \sum_{j=1}^{N_{f, i}}\left(T_{f, j}^{*}-T_{f, i}^{*}\right) \operatorname{Tr}\left(\mathbf{g}_{2}\left(\mathbf{x}_{f, j}^{*}-\mathbf{x}_{f, i}^{*}\right)\right) V_{f, j}^{*} \\
& -\sum_{j=1}^{N_{f, i}}\left(T_{f, j}^{*}-T_{f, i}^{*}\right)\left(\mathbf{v}_{f, i}^{*} \cdot \mathbf{g}_{1}\left(\mathbf{x}_{f, j}^{*}-\mathbf{x}_{f, i}^{*}\right)\right) V_{f, j}^{*}
\end{aligned}
$$


The energy dissipated by the viscous force is ignored in Eq.(58) since it is too small compared to the energy increased by fire [88]. The non-dimensional parameters shown with $\left({ }^{*}\right)$ are defined with respect to their reference values shown with $\left({ }_{0}\right)$ as [89]

$$
\mathbf{x}_{f}^{*}=\frac{\mathbf{x}_{f}}{L_{0}} ; \mathbf{v}_{f}^{*}=\frac{\mathbf{v}_{f}}{v_{0}} ; t^{*}=\frac{t}{\left(L_{0} / v_{0}\right)} ; \rho_{f}^{*}=\frac{\rho_{f}}{\rho_{f, 0}} ; P_{f}^{*}=\frac{P_{f}}{\rho_{f} v_{0}^{2}} ; T_{f}^{*}=\frac{T_{f}}{T_{0}}, \mu_{f}^{*}=\frac{\mu_{f}}{\mu_{f, 0}}, k_{f}^{*}=\frac{k_{f}}{k_{f, 0}}(
$$

where the parameters $\mathbf{x}_{f}, \mathbf{v}_{f}, \rho_{f}, P_{f}, T_{f}, \mu_{f}$, and $k_{f}$ represent the fluid point position, velocity, density, pressure, temperature, viscous coefficient, and thermal conductivity. $L_{0}, v_{0}$, and $T_{0}$ are the reference length, velocity, and temperature. $\rho_{f, 0}, \mu_{f, 0}$, and $k_{f, 0}$ are the reference fluid density, viscosity and thermal conductivity. $t$ and $t^{*}$ represent the time and non-dimensional time, respectively.

In Eqs.(56)-(58), the fluid point of interested is denoted by its coordinate as $\mathbf{x}_{f, i}^{*}$, and its family member is denoted by $\mathbf{x}_{f, j}^{*} . N_{f, i}$ is the total number of fluid family members of point $\mathbf{x}_{f, i}^{*}$. The volume of $\mathbf{x}_{f, j}^{*}$ is denoted by $V_{f, j}^{*}$. Tr represents the trace of a matrix. The parameters $\mathbf{v}_{f, i}^{*}, \rho_{f, i}^{*}, \mu_{f, i}^{*}, T_{f, i}^{*}$, and $k_{f, i}^{*}$ are defined at location $\mathbf{x}_{f, i}^{*}$

The non-dimensional parameters Prandtl number Pr, and Rayleigh number Ra in Eq. (58) are defined as [90]

$$
\operatorname{Pr}=\frac{\mu_{f, 0} c_{f}}{k_{f, 0}}, \operatorname{Ra}=\frac{g \beta_{f} \Delta T L_{0}^{3} \rho_{f, 0}^{2} c_{f}}{\mu_{f, 0} k_{f, 0}}
$$

where the parameters $c_{f}$ and $\beta_{f}$ are the specific heat capacity and the thermal expansion coefficient of the fluid. The term $g$ represents the magnitude of the gravitational acceleration. The parameter $\Delta T$ represents the temperature difference across length $L_{0}$.

In Eqs.(56)-(58), $\mathbf{g}_{1}\left(\xi^{*}\right)$ and $\mathbf{g}_{2}\left(\xi^{*}\right)$ denotes the PDDO matrices as [78]

$$
\mathbf{g}_{1}\left(\xi^{*}\right)=\left[\begin{array}{ll}
g^{10}\left(\xi^{*}\right) & g^{01}\left(\xi^{*}\right)
\end{array}\right]
$$

and 


$$
\mathbf{g}_{2}\left(\xi^{*}\right)=\left[\begin{array}{ll}
g^{20}\left(\xi^{*}\right) & g^{11}\left(\xi^{*}\right) \\
g^{11}\left(\xi^{*}\right) & g^{02}\left(\xi^{*}\right)
\end{array}\right]
$$

where $g^{10}(\xi), g^{01}(\xi), g^{20}(\xi), g^{02}(\xi), g^{11}(\xi)$ represent the peridynamic operators [74] and $\xi^{*}$ represents the relative position between fluid points $\mathbf{x}_{f, i}^{*}$ and $\mathbf{x}_{f, j}^{*}$ as $\xi^{*}=\mathbf{x}_{f, j}^{*}-\mathbf{x}_{f, i}^{*}$. The peridynamic operators are defined as [74]

$$
\left\{\begin{array}{l}
g^{10}\left(\xi^{*}\right) \\
g^{01}\left(\xi^{*}\right) \\
g^{20}\left(\xi^{*}\right) \\
g^{02}\left(\xi^{*}\right) \\
g^{11}\left(\xi^{*}\right)
\end{array}\right\}=\left(\mathbf{A}_{P D D O}^{-1} \mathbf{b}_{\text {PDDO }}\right)^{\text {Transpose }}\left\{\begin{array}{c}
w \xi_{x}^{*} \\
w \xi_{y}^{*} \\
w\left(\xi_{x}^{*}\right)^{2} \\
w\left(\xi_{y}^{*}\right)^{2} \\
w \xi_{x}^{*} \xi_{y}^{*}
\end{array}\right\}
$$

where

$$
\begin{aligned}
& \xi^{*}=\left[\begin{array}{ll}
\xi_{x}^{*} & \xi_{y}^{*}
\end{array}\right] \\
& w=e^{-\left(2\left|\xi^{*}\right| / \delta^{*}\right)^{2}} \\
& \mathbf{A}_{P D D O}=\sum_{j=1}^{N_{f, i}} w\left[\begin{array}{ccccc}
\left(\xi_{x}^{*}\right)^{2} & \xi_{x}^{*} \xi_{y}^{*} & \left(\xi_{x}^{*}\right)^{3} & \xi_{x}^{*}\left(\xi_{y}^{*}\right)^{2} & \left(\xi_{x}^{*}\right)^{2} \xi_{y}^{*} \\
\xi_{x}^{*} \xi_{y}^{*} & \left(\xi_{y}^{*}\right)^{2} & \left(\xi_{x}^{*}\right)^{2} \xi_{y}^{*} & \left(\xi_{y}^{*}\right)^{3} & \xi_{x}^{*}\left(\xi_{y}^{*}\right)^{2} \\
\left(\xi_{x}^{*}\right)^{3} & \left(\xi_{x}^{*}\right)^{2} \xi_{y}^{*} & \left(\xi_{x}^{*}\right)^{4} & \left(\xi_{x}^{*}\right)^{2}\left(\xi_{y}^{*}\right)^{2} & \left(\xi_{x}^{*}\right)^{3} \xi_{y}^{*} \\
\xi_{x}^{*}\left(\xi_{y}^{*}\right)^{2} & \left(\xi_{y}^{*}\right)^{3} & \left(\xi_{x}^{*}\right)^{2}\left(\xi_{y}^{*}\right)^{2} & \left(\xi_{y}^{*}\right)^{4} & \xi_{x}^{*}\left(\xi_{y}^{*}\right)^{3} \\
\left(\xi_{x}^{*}\right)^{2} \xi_{y}^{*} & \xi_{x}^{*}\left(\xi_{y}^{*}\right)^{2} & \left(\xi_{x}^{*}\right)^{3} \xi_{y}^{*} & \xi_{x}^{*}\left(\xi_{y}^{*}\right)^{3} & \left(\xi_{x}^{*}\right)^{2}\left(\xi_{y}^{*}\right)^{2}
\end{array}\right] V_{f} \\
& \mathbf{b}_{P D D O}=\left[\begin{array}{ccccc}
1 & 0 & 0 & 0 & 0 \\
0 & 1 & 0 & 0 & 0 \\
0 & 0 & 2 & 0 & 0 \\
0 & 0 & 0 & 2 & 0 \\
0 & 0 & 0 & 0 & 1
\end{array}\right]
\end{aligned}
$$

The fluid is treated as weakly compressible, where the pressure of fluid point $\mathbf{x}_{f, i}^{*}$ is calculated via the equation of state as [90] 


$$
P_{f, i}^{*}=\left(\frac{c_{\text {sound }}}{v_{0}}\right)^{2}\left(\left(\rho_{f, i}^{*}\right)^{\gamma_{f}}-1\right)
$$

where $\gamma_{f}$ is the ratio of specific heat capacity, $c_{\text {sound }}$ is the artificial speed of sound. The ratio of specific heat capacity is chosen as $\gamma_{f}=1$ for gas [90].

Furthermore, Sutherland's law [91-93] which reflects the temperature effect on the viscous coefficient and the thermal conductivity is adopted in a non-dimensional form as

$$
\mu_{f, i}^{*}=\left(T_{f, i}^{*}\right)^{1.5} \frac{1+S^{*}}{T_{f, i}^{*}+S^{*}} ; \quad k_{f, i}^{*}=\left(T_{f, i}^{*}\right)^{1.5} \frac{1+S^{*}}{T_{f, i}^{*}+S^{*}} ;
$$

where $S^{*}=111 / T_{0}$ with $T_{0}$ being in unit K [91].

\subsection{Non-dimensional PD composite thermal model}

The PD thermo-fluid model given in Section 3.1 is in non-dimensional form. Therefore, in order to couple the thermal-fluid model and the composite model, the non-dimensional form of the PD composite thermal model is used. It should be noted that the length, time, and temperature are cast into their non-dimensional values by using the same reference parameters both for the composite model and the fluid model. Furthermore, the temperature in the fitting functions of thermal degradation properties of composites is in a dimensional form. Therefore, the non-dimensional temperature should be converted into its dimensional value before the calculation of the composite material properties.

According to Eq.(59), the non-dimensional parameters for PD composite model are defined as

$$
\mathbf{x}_{s}^{*}=\frac{\mathbf{x}_{s}}{L_{0}} ; \mathbf{v}_{s}^{*}=\frac{\mathbf{v}_{s}}{v_{0}} ; T_{s}^{*}=\frac{T_{s}}{T_{0}} ; t^{*}=\frac{t}{\left(L_{0} / v_{0}\right)}
$$

where the values of $L_{0}, v_{0}$, and $T_{0}$ are the reference values used in Eq.(59). For both the composite and the fluid PD model, the reference velocity is chosen as

$$
v_{0}=\frac{\alpha_{d f, 0}}{L_{0}}
$$

where $\alpha_{d f, 0}$ is the initial value of the thermal diffusivity of air as 


$$
\alpha_{d f, 0}=\frac{k_{f, 0}}{\rho_{f, 0} c_{f}}
$$

Consequently, the nondimensional diffusivities of composites to the fluid are calculated as

$$
\alpha_{d s 1}^{*}=\frac{\alpha_{d s 1}}{\alpha_{d f, 0}} ; \alpha_{d s 2}^{*}=\frac{\alpha_{d s 2}}{\alpha_{d f, 0}} ; \alpha_{d s m}^{*}=\frac{\alpha_{d s m}}{\alpha_{d f, 0}}
$$

where $\alpha_{d s 1}$ is the thermal diffusivity in the fibre direction, $\alpha_{d s 2}$ is the thermal diffusivity in the transverse direction, and $\alpha_{d s m}$ is the thermal diffusivity of the matrix in the composite material. By substituting Eqs.(70)-(73) into Eq.(51), the non-dimensional form of the PD composite thermal model becomes

$$
\begin{aligned}
& \frac{\partial T_{s, i}^{n, *}}{\partial t^{*}} \\
& =\sum_{j=1}^{N_{s, p l y, i}}\left[\left(\mu_{f} \chi_{F} \frac{\kappa_{F, i}^{n, *}+\kappa_{F, j}^{n, *}}{2}+\mu_{m} \frac{\kappa_{m, i}^{n, *}+\kappa_{m, j}^{n, *}}{2}\right) \frac{\left(T_{s, j}^{n, *}-T_{s, i}^{n, *}\right)}{\left|\mathbf{x}_{s, j}^{n, *}-\mathbf{x}_{s, i}^{n, *}\right|}\right] V_{s, j}^{n, *} \\
& +\sum_{m=n+1, n-1}\left[\mu_{i n} \kappa_{\text {inter,i }}^{n, *} \frac{\left(T_{s, i}^{m, *}-T_{s, i}^{n, *}\right)}{\left|\mathbf{x}_{s, i}^{*, m}-\mathbf{x}_{s, i}^{n, *}\right|}\right] V_{s, i}^{m, *}+\sum_{m=n+1, n-1} \sum_{j=1}^{N_{s, s h e a r, i}}\left[\mu_{i s} \kappa_{\text {inter }, i}^{n, *} \frac{\left(T_{s, j}^{m, *}-T_{s, i}^{n, *}\right)}{\left|\mathbf{x}_{s, j}^{*, m}-\mathbf{x}_{s, i}^{n, *}\right|}\right] V_{s, j}^{m, *}+h_{s, i}^{n, *}
\end{aligned}
$$

By using Eqs.(52)-(54), the non-dimensional PD thermal diffusivities are defined as

$$
\begin{aligned}
& \kappa_{F, i}^{n, *}=\frac{2\left(\alpha_{d s 1}^{*}-\alpha_{d s 2, i}^{*}\right)}{\sum_{j=1}^{N_{s, f i b r e, i}}\left|\mathbf{x}_{s, j}^{n, *}-\mathbf{x}_{s, i}^{n, *}\right| V_{s, j}^{n, *}} \\
& \kappa_{m, i}^{n * *}=\frac{6 \alpha_{d s 2, i}^{*}}{\pi h_{t h i c k}^{*}\left(\delta^{*}\right)^{3}} \\
& \kappa_{\text {inter }, i}^{n * *}=\frac{\alpha_{d s m}^{*}}{2 \pi\left(h_{t h i c k}^{*}\right)^{3}\left(\tilde{\delta}^{*}-h_{\text {thick }}^{*}\right)}
\end{aligned}
$$

where the parameters $\kappa_{F, i}^{n, *}, \kappa_{m, i}^{n, *}$, and $\kappa_{\text {inter,i }}^{n, *}$ are defined at location $\mathbf{x}_{s, i}^{n, *}$.

In Eq. (74), $h_{s, i}^{*}$ is the non-dimensional volumetric heat source, which can be represented as 


$$
h_{s, i}^{n, *}=\frac{h_{s, i}^{n} L_{0}}{\rho_{s} C_{v} T_{0} v_{0}}
$$

\section{Numerical implementations}

The PD models in Section 2 and Section 3 are programmed by using Fortran programming language. The Math Kernel Library (MKL) [94] is utilized to calculate the PDDO. The following contents provide detailed information about the numerical implementations.

\subsection{Governing equations in discretised form}

The thermo-fluid governing equations in the discretised form are (according to Eq.(56)-(58)):

Conservation of mass:

$$
\begin{aligned}
& \rho_{f, i}^{*(\mathrm{n}+1)}=\rho_{f, i}^{*(\mathrm{n})} \\
& -\Delta t^{*}\left(\begin{array}{l}
\rho_{f, i}^{*(\mathrm{n})} \sum_{j=1}^{N_{f, i}}\left(\mathbf{v}_{f, j}^{*(\mathrm{n})}-\mathbf{v}_{f, i}^{*(\mathrm{n})}\right) \cdot \mathbf{g}_{1}\left(\mathbf{x}_{f, j}^{*}-\mathbf{x}_{f, i}^{*}\right) V_{f, j}^{*} \\
-\sum_{j=1}^{N_{f, i}}\left(\rho_{f, j}^{*(\mathrm{n})}-\rho_{f, i}^{*(\mathrm{n})}\right)\left(\mathbf{v}_{f, i}^{*(\mathrm{n})} \cdot \mathbf{g}_{1}\left(\mathbf{x}_{f, j}^{*}-\mathbf{x}_{f, i}^{*}\right)\right) V_{f, j}^{*}
\end{array}\right)
\end{aligned}
$$

Conservation of momentum:

$$
\begin{aligned}
& \mathbf{v}_{f, i}^{*(\mathrm{n}+1)}=\mathbf{v}_{f, i}^{*(\mathrm{n})} \\
& +\Delta t^{*}\left(\begin{array}{l}
-\sum_{j=1}^{N_{f, i}}\left(P_{f, j}^{*(\mathrm{n})}-P_{f, i}^{*(\mathrm{n})}\right) \mathbf{g}_{1}\left(\mathbf{x}_{f, j}^{*}-\mathbf{x}_{f, i}^{*}\right) V_{f, j}^{*} \\
+\sum_{j=1}^{N_{f, i}}\left(\mathbf{v}_{f, j}^{*(\mathrm{n})}-\mathbf{v}_{f, i}^{*(\mathrm{n})}\right)\left(\begin{array}{l}
\operatorname{Pr} \mu_{f, i}^{*(\mathrm{n})} \operatorname{Tr}\left(\mathbf{g}_{2}\left(\mathbf{x}_{f, j}^{*}-\mathbf{x}_{f, i}^{*}\right)\right) \\
-\left(\mathbf{v}_{f, i}^{*(\mathrm{n})} \cdot \mathbf{g}_{1}\left(\mathbf{x}_{f, j}^{*}-\mathbf{x}_{f, i}^{*}\right)\right)
\end{array}\right) V_{f, j}^{*} \\
+\operatorname{Ra} \operatorname{Pr} T_{f, i}^{*(\mathrm{n})}\left[\begin{array}{l}
0 \\
1
\end{array}\right]
\end{array}\right)
\end{aligned}
$$

Conservation of energy:

$$
\begin{aligned}
& T_{f, i}^{*(\mathrm{n}+1)}=T_{f, i}^{*(\mathrm{n})}+ \\
& \Delta t^{*} \sum_{j=1}^{N_{f, i}}\left(T_{f, j}^{*(\mathrm{n})}-T_{f, i}^{*(\mathrm{n})}\right)\left(\begin{array}{c}
k_{f, i}^{*(\mathrm{n})} \operatorname{Tr}\left(\mathbf{g}_{2}\left(\mathbf{x}_{f, j}^{*}-\mathbf{x}_{f, i}^{*}\right)\right) \\
-\left(\mathbf{v}_{f, i}^{*(\mathrm{n})} \cdot \mathbf{g}_{1}\left(\mathbf{x}_{f, j}^{*}-\mathbf{x}_{f, i}^{*}\right)\right)
\end{array}\right) V_{f, j}^{*}
\end{aligned}
$$


pressure:

$$
P_{f, i}^{*(\mathrm{n}+1)}=\left(\frac{c_{\text {sound }}}{v_{0}}\right)^{2}\left(\left(\rho_{f, i}^{*(\mathrm{n}+1)}\right)^{\gamma_{f}}-1\right)
$$

The thermal model for the composite is (according to Eq.(74)):

$$
\begin{aligned}
& T_{s, i}^{n, *(\mathrm{n}+1)}=T_{s, i}^{n, *(\mathrm{n})} \\
& +\Delta t^{*}\left(\begin{array}{l}
\sum_{j=1}^{N_{s, p l y, i}}\left[\left(\mu_{f} \chi_{F} \frac{\kappa_{F, i}^{n, *(\mathrm{n})}+\kappa_{F, j}^{n, *(\mathrm{n})}}{2}+\mu_{m} \frac{\kappa_{m, i}^{n, *(\mathrm{n})}+\kappa_{m, j}^{n, *(\mathrm{n})}}{2}\right) \frac{\left(T_{s, j}^{n, *(\mathrm{n})}-T_{s, i}^{n, *(\mathrm{n})}\right)}{\left|\mathbf{x}_{s, j}^{n, *}-\mathbf{x}_{s, i}^{n, *}\right|}\right] V_{s, j}^{n, *} \\
+\sum_{m=n+1, n-1}\left[\mu_{i n} \kappa_{\mathrm{int} e r, i}^{n, *(\mathrm{n})} \frac{\left(T_{s, i}^{m, *(\mathrm{~m})}-T_{s, i}^{n, *(\mathrm{n})}\right)}{\left|\mathbf{x}_{s, i}^{*, m}-\mathbf{x}_{s, i}^{n, *}\right|}\right] V_{s, i}^{m, *}+ \\
\quad \sum_{m=n+1, n-1} \sum_{j=1}^{N_{s, s h a a r, i}}\left[\mu_{i s} \kappa_{\mathrm{int} e r, i}^{n, *(\mathrm{n})} \frac{\left(T_{s, j}^{m, *(\mathrm{~m})}-T_{s, i}^{n, *(\mathrm{n})}\right)}{\left|\mathbf{x}_{s, j}^{*, m}-\mathbf{x}_{s, i}^{n, *}\right|}\right] V_{s, j}^{m, *}+h_{s, i}^{n, *(\mathrm{n})}
\end{array}\right)
\end{aligned}
$$

In the above equations, the current time step is denoted by the superscript $\left({ }^{(n)}\right)$ and the updated time step is denoted by the superscript $\left({ }^{(n+1)}\right)$. The non-dimensional time step size is denoted by $\Delta t^{*}$.

\subsection{Boundary conditions}

\subsubsection{Mechanical boundary conditions}

The boundary condition involved in the composite mechanical model is applied by setting displacement values in the fictitious layer as [47]

$$
\mathbf{u}_{s, b c}=0
$$

In Fig. 11, $R_{s}$ represents the composite material, and $R_{s, b c}$ represents the fictitious region. The thickness of the fictitious layer is set as the size of the horizon (shown in black points in Fig. 11 (b)). 


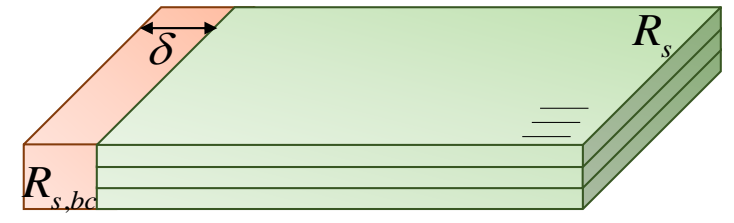

(a)

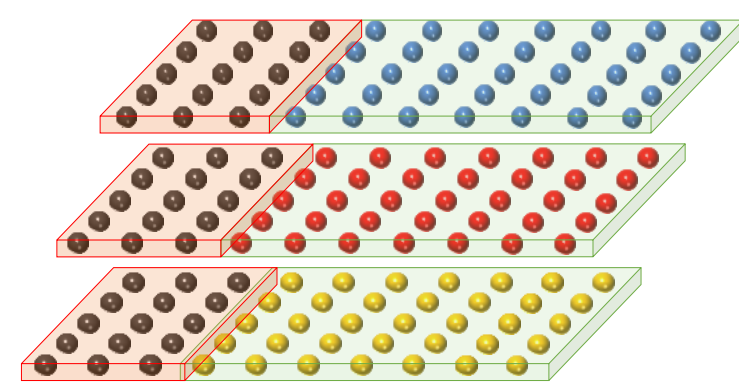

(b)

Fig. 11 Fixed boundary implementation (a) geometry illustration and (b) PD implementation for $\delta=3 \Delta x$

\subsubsection{Flow boundary conditions}

The boundary condition involved in the fluid field is applied by setting non-dimensional velocity and displacement values in the fictitious layer as

$$
\mathbf{v}_{f}^{*}\left(\mathbf{x}_{f, b c}^{*}\right)=0 ; \mathbf{u}_{f}^{*}\left(\mathbf{x}_{f, b c}^{*}\right)=0
$$

The pressure values at the fictitious points in $R_{f, b c}$ are defined in a non-dimensional form as $[48,77]$

$$
P_{f}^{*}\left(\mathbf{x}_{f, b c}^{*}, t^{*}\right)=\frac{\sum_{j=1}^{N_{f, b c}} P_{f}^{*}\left(\mathbf{x}_{f, j}^{*}, t^{*}\right) w\left(\mathbf{x}_{f, j}^{*}-\mathbf{x}_{f, b c}^{*}\right)}{\sum_{j=1}^{N_{f, b c}} w\left(\mathbf{x}_{f, j}^{*}-\mathbf{x}_{f, b c}^{*}\right)}
$$

where $\mathbf{x}_{f, b c}^{*}$ represents the fictitious rigid wall point, $\mathbf{x}_{f, j}^{*}$ is its family member belonging to the fluid. $N_{f, b c}$ represents the total number of family members of point $\mathbf{x}_{f, b c}^{*}$ belonging to the fluid field, as shown in Fig. 12. $P_{f}^{*}\left(\mathbf{x}_{f, j}^{*}, t^{*}\right)$ represents the pressure of point $\mathbf{x}_{f, j}^{*}$. The weighted function $w$ provided in Eq.(65) is used. 


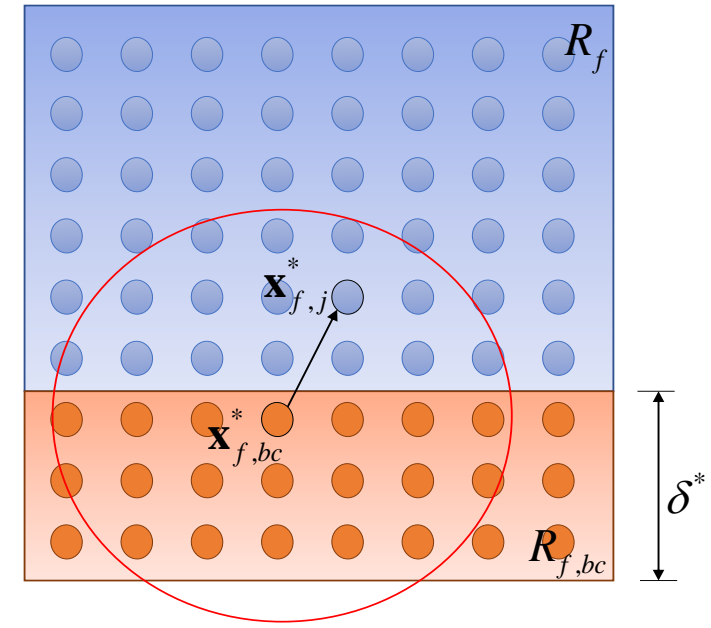

Fig. 12 Boundary condition implementation for fluid field with $\delta^{*}=3 \Delta x^{*}$

\subsubsection{Thermal boundary conditions}

The thermal boundary conditions are both involved in the composite model and fluid model. The temperature boundary condition is applied by adding fictitious layers as $[57,95]$

$$
T_{i}^{*}\left(t^{*}\right)=2 T_{b c}^{*}\left(t^{*}\right)-T_{j}^{*}\left(t^{*}\right)
$$

where $T_{b c}^{*}$ represents the non-dimensional boundary temperature. The parameters $T_{i}^{*}$ and $T_{j}^{*}$ represent the non-dimensional temperatures in the fictitious and real regions, respectively, as shown in Fig. 13.

No flux (insulated) boundary condition is applied by setting temperatures in the real region as [57, 95]

$$
T_{j}^{*}\left(t^{*}\right)=T_{k}^{*}\left(t^{*}\right)
$$




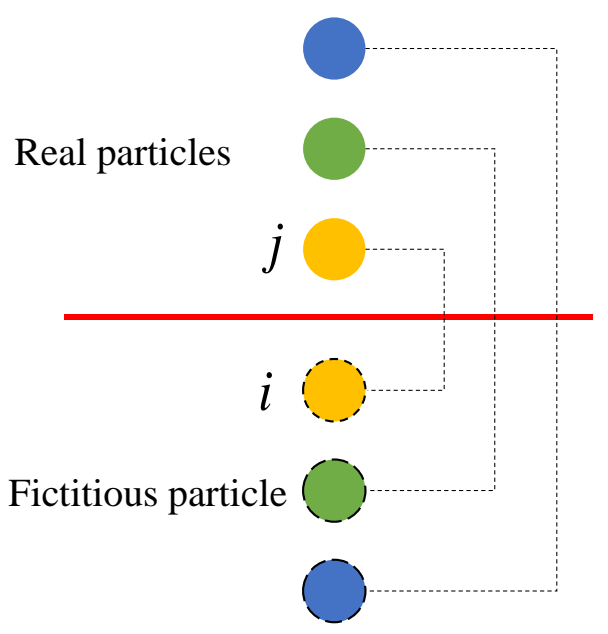

(a)

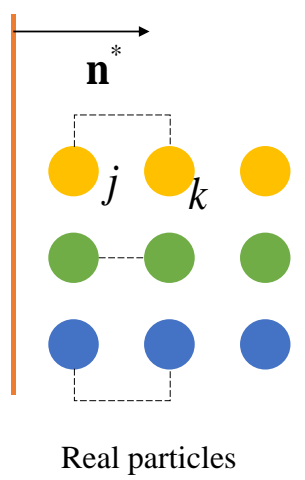

(b)

Fig. 13 Thermal boundary condition implementation (a) temperature boundary condition, and (b) insulated boundary condition

\subsection{Interface condition between composite and gas for thermal field}

The convection boundary condition at the composite is implemented by setting heat source at the boundary points as $[57,95]$

$$
h_{s}^{*}\left(\mathbf{x}_{s, \text { interface }}^{*}, t^{*}\right)=-\frac{1}{\Delta x^{*}} h_{\text {con }}^{*}\left(T_{s}^{*}\left(\mathbf{x}_{s, \text { interface }}^{*}, t^{*}\right)-T_{f, \text { local }}^{*}\right)
$$

where $h_{c o n}^{*}$ is the non-dimensional convective heat transfer coefficient, $T_{s}^{*}\left(\mathbf{x}_{s, \text { interface }}^{*}, t^{*}\right)$ is the temperature in the composite region, and $T_{f, l o c a l}^{*}$ is the local fluid temperature which is calculated as the average temperature of the fluid points which are within the horizon of $\mathbf{x}_{s, \text { interface }}^{*}$ as

$$
T_{f, \text { local }}^{*}=\frac{\sum_{N_{\text {sinteface, } f}} T_{f}^{*}\left(\mathbf{x}_{f}^{*}, t^{*}\right)}{N_{\text {s,interface }, f}}
$$

where $N_{\text {s,interface, }}$ represents the total number of the family members of point $\mathbf{x}_{s, \text { interface }}^{*}$ which belongs to the fluid as shown in Fig. 14. $T_{f}^{*}\left(\mathbf{x}_{f}^{*}, t^{*}\right)$ is the non-dimensional fluid temperature predicted by the fluid model. 


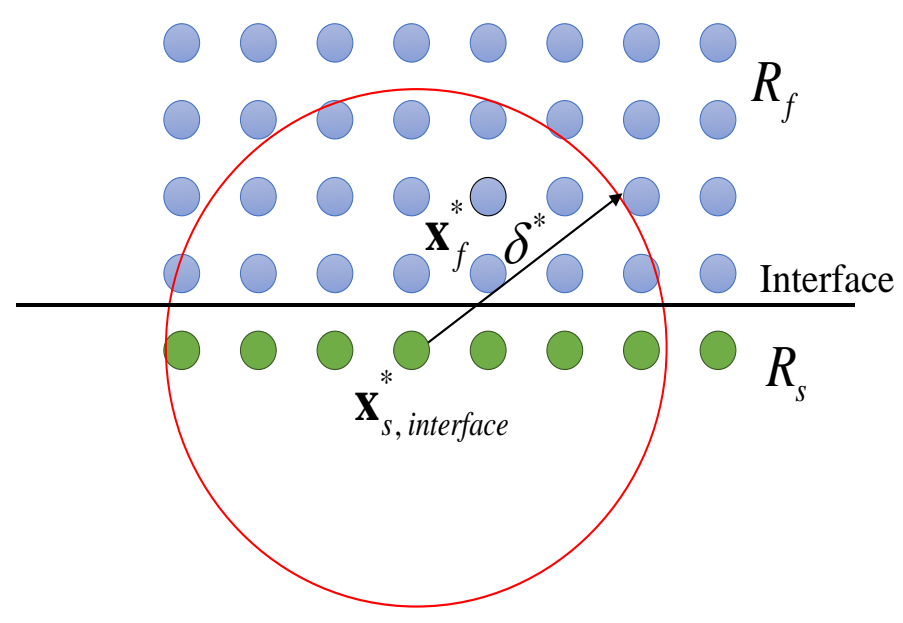

Fig. 14 Composite-gas interface illustration

The non-dimensional convection coefficient $[93,96]$ is

$$
h_{\text {con }}^{*}=\max \left(\frac{C_{\text {convection }} T_{0}^{1 / 3}\left|T_{s}^{*}\left(\mathbf{x}_{s, \text { interface }}^{*}, t^{*}\right)-T_{f, i}^{*}\right|^{1 / 3}}{\rho_{s} c_{v} v_{0}}, \frac{k_{f, 0}}{\rho_{s} c_{v} \alpha_{f, 0}} \frac{k_{f}^{*}}{L_{s}^{*}} 0.0037 \operatorname{Re}^{0.8} \operatorname{Pr}^{1 / 3}\right)
$$

where $C_{\text {convection }}$ is a coefficient for natural convection which is $C_{\text {convection }}=1.52 \mathrm{~W} /\left(\mathrm{m}^{2} \mathrm{~K}^{4 / 3}\right)$ for a horizontal surface and $C_{\text {convection }}=1.38 \mathrm{~W} /\left(\mathrm{m}^{2} \mathrm{~K}^{4 / 3}\right)$ for a vertical surface. The parameter $L_{s}^{*}$ is the non-dimensional length of the composite ply. $c_{v}$ is the specific heat capacity of composites provided in Eq.(3).

Similarly, the radiation boundary condition for the composite thermal field is implemented by setting heat source at the boundary as [57]

$$
h_{s}^{*}\left(\mathbf{x}_{\text {s,interface }}^{*}, t^{*}\right)=\sigma_{\text {stefan-boltzmann }}^{*} \varepsilon \frac{1}{\Delta x^{*}}\left(\left(T_{f, \text { local }}^{*}\right)^{4}-\left(T_{s}\left(\mathbf{x}_{s, \text { interface }}^{*}, t^{*}\right)\right)^{4}\right)
$$

where $\sigma_{\text {stefan-boltzmann }}^{*}$ is the non-dimensional Stefan Boltzman constant defined as

$$
\sigma_{\text {stefan-boltzmann }}^{*}=\frac{\sigma_{\text {stefan-boltzmann }} T_{0}^{3} L_{0}}{\rho_{s} c_{v} \alpha_{f, 0}}
$$

with $\sigma_{\text {stefan-boltzmann }}=5.67 \times 10^{-8} \mathrm{~W} /\left(\mathrm{m}^{2} \mathrm{~K}^{4}\right)$. The surface emissivity $\varepsilon$ is assumed to be one as $\varepsilon=1$ in the current study. 


\section{Numerical simulations}

In this section, two cases are presented. The first case investigates the damage in composite due to direct contact with fire. This case belongs to the thermomechanical analysis where the heat conduction and composite deformation are included. The second case investigates the damage in composite due to hot gas induced by fire. Fire is simulated as a temperature boundary condition which heats the air. Then the heated air transfers the heat energy to the composite material via convection and radiation, indicating a one-way coupling thermal fluid and structure interaction.

\subsection{Composite damage due to direct contact with the fire}

5.1.1. Problem description

As illustrated in Fig. 15, a three-layer laminated composite material is investigated. The laminate is $0.1 \mathrm{~m}$ in length $(L=0.1 \mathrm{~m})$ and $0.1 \mathrm{~m}$ in width $(\mathrm{W}=0.1 \mathrm{~m})$. Each layer has a uniform thickness as $h_{\text {thick }}=0.001 \mathrm{~m}$. The fibre direction of each layer is chosen as $\left[0 / 90^{\circ} / 0\right]$. The composite material properties $k_{1}, k_{2}, c_{v}, E_{1}, E_{2}, G_{12}, \alpha_{1}, \alpha_{2}, G_{I C}, K_{m}, \mu_{m}, v_{12}, \alpha_{m}, E_{m}$, and $G_{m}$ are provided in Section 2.2. The density of the composite is $\rho_{s}=1620 \mathrm{~kg} / \mathrm{m}^{3}$.

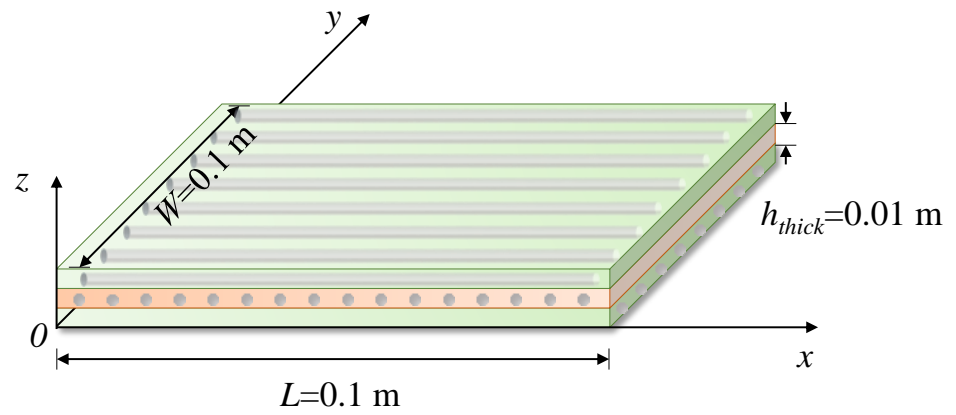

(a)

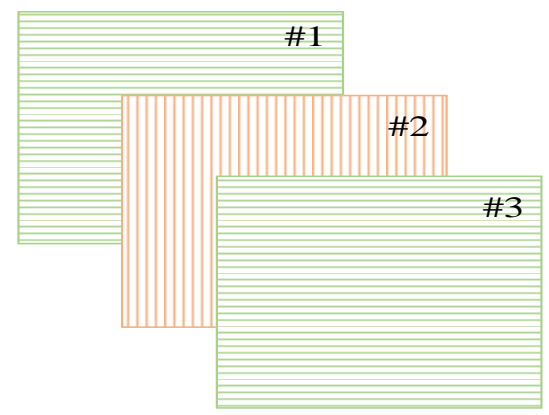

(b)

Fig. 15 (a) Three layers laminate geometry illustration and (b) fibre direction illustration

A uniform initial temperature is set for the composite as $T_{s}^{0}=T_{s, r e f}=293 \mathrm{~K}$. Besides, all composite points are static at the initial condition. The boundary conditions are provided as follows. 
Thermal boundary conditions:

As shown in Fig. 16, the edge on $x=0,|y-W / 2|<W / 8$ is under a temperature boundary condition induced by a localized fire. The other three edges $x=L, y=0$, and $y=W$ are insulated.

At $x=0$

$$
\begin{aligned}
& |y-W / 2|<W / 8: T_{s}=T_{\text {fire }}(t)=345 \log (8 t / 60+1)+T_{s}^{0} \\
& |y-W / 2| \geq W / 8: \frac{\partial T_{s}}{\partial x}=0
\end{aligned}
$$

At $x=L$

$$
\frac{\partial T}{\partial x}=0
$$

At $y=0$ and $y=W$

$$
\frac{\partial T}{\partial y}=0
$$

Mechanical boundary conditions:

As to the mechanical field, the right vertical edge is fixed, and the other three edges are free to deform as

At $x=L$

$$
\mathbf{u}_{s}=0
$$




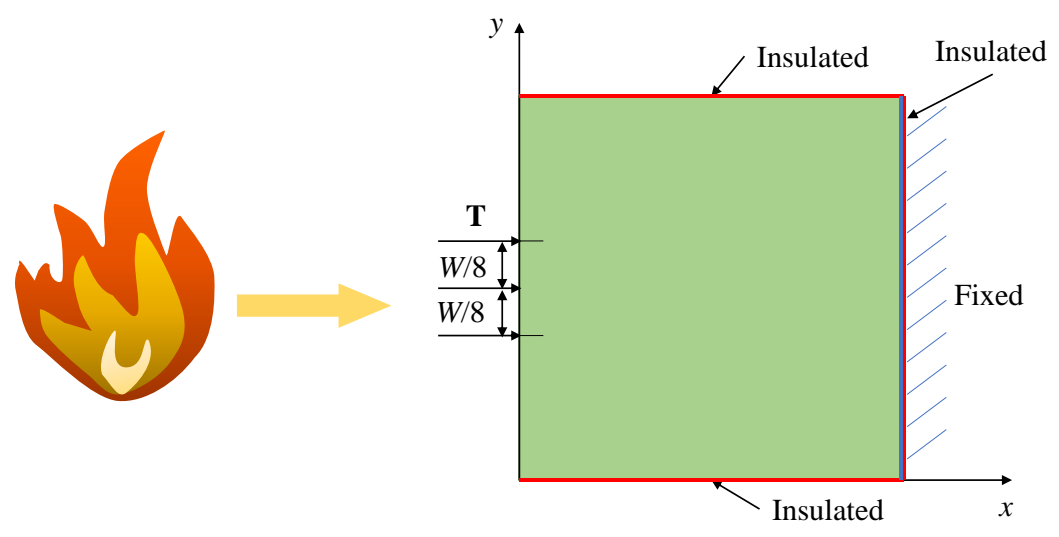

(a)

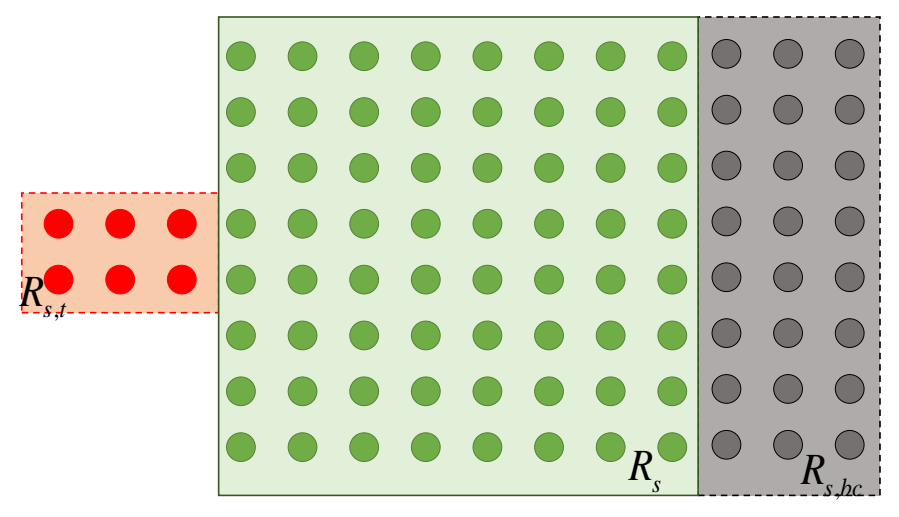

(b)

Fig. 16 Boundary sketch for composite directly under local fire (top view): (a) geometry illustration and (b) PD discretization

The top view of the PD discretization is illustrated in Fig. 16 (b). For the laminate ply, denoted by $R_{s}$, each layer is discretized by $40 \times 40$ points in the $x-y$ plane and one point in the $z$ direction. Therefore, the laminate totally contains $40 \times 40 \times 3$ PD points and the initial inplane point spacing is $\Delta x=0.0025 \mathrm{~m}$. The horizon size is chosen as $\delta=3.015 \Delta x$ for the inplane deformations for the composite model as suggested in [47, 48, 63]. The region denoted by $R_{s, t}$ is the thermal fictitious layer $(10 \times 3 \times 3 \mathrm{PD}$ points $)$ and $R_{s, b c}$ is the fictitious layer $(40 \times 3 \times 3$ PD points) representing the fixed boundary condition in the mechanical field. The initial values of the critical stretches are $s_{c m}=3.39 \times 10^{-3}, s_{c f}=1.99 \times 10^{-2}, s_{c i n}=1.97 \times 10^{-2}$, and $s_{c i s}=2.25 \times 10^{-2}$ which are calculated based on parameters $\delta_{1 t}, E_{1}, G_{I C}, \mu_{m}, K_{m}, E_{m}$, and $G_{m}$ as provided in Section 2.2 and Section 2.3.

The time step size for heat conduction analysis is set as $\Delta t=10^{-3} \mathrm{~s}$. The mechanical deformation analysis is conducted in a quasi-static solution for each 50 seconds by using the 
adaptive dynamic relaxation (ADR) technique [97] with iteration time step $N_{A D R}=8000$. The total simulation time is $500 \mathrm{~s}$.

\subsubsection{Numerical procedure}

In this thermomechanical analysis, the heat conduction is performed in a transient process, while the mechanical analysis is performed as a static case for each 50 seconds. The numerical procedure for the thermomechanical problem 5.1 is provided in Fig. 17. 


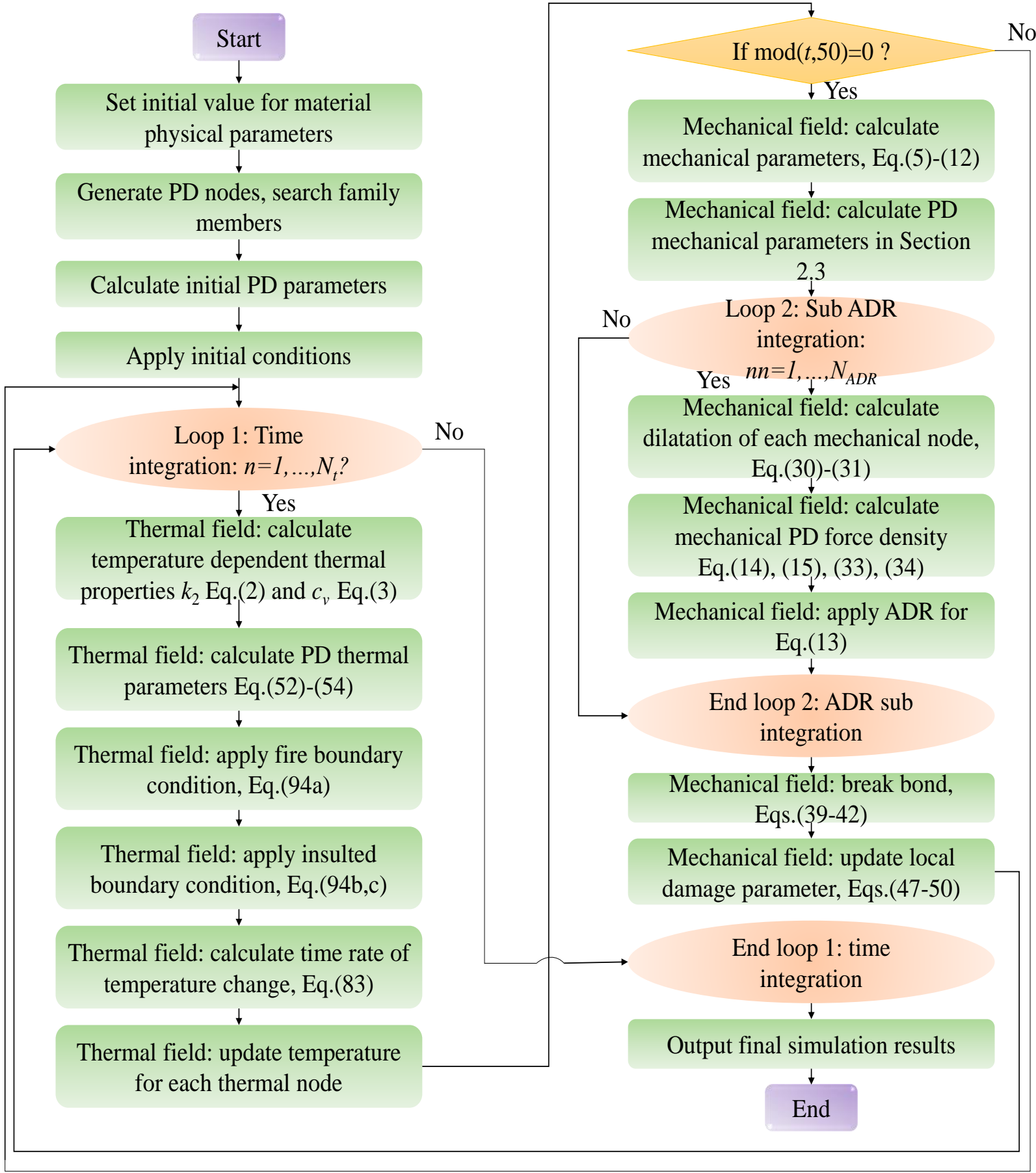

Fig. 17 Flowchart for thermo-mechanical model for composite for problem 5.1

\subsubsection{Numerical results}

The temperature change distributions of the laminate at different times are provided in Fig. 18. It can be observed that the temperature change distributions for the bottom and top plies are identical but differ from the distribution for the middle ply. The fibre direction which has a larger thermal conductivity is parallel to $x$ direction for the bottom and top plies, leading to a faster heat transfer in the $x$ direction compared to the middle ply. Therefore, the heated region 
of the top and bottom plies in the figures are more concentrated in the $x$ direction than the middle ply.

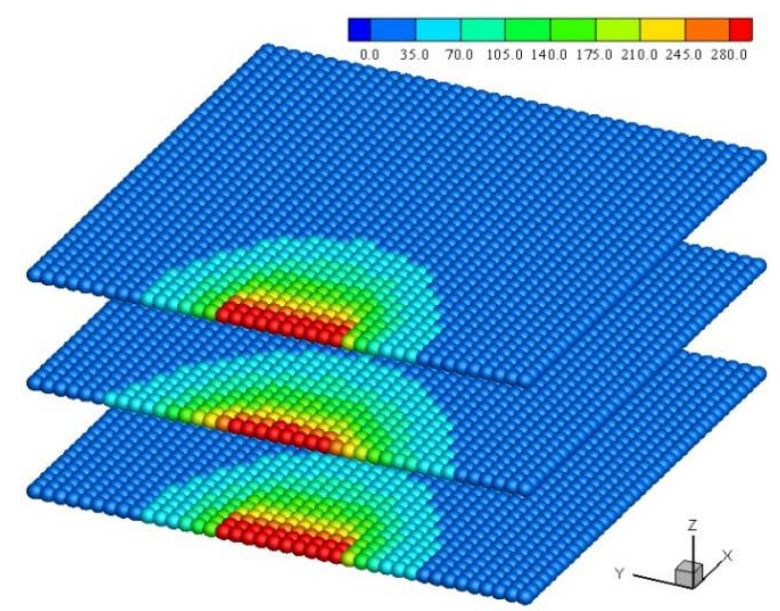

(a) $100 \mathrm{~s}$

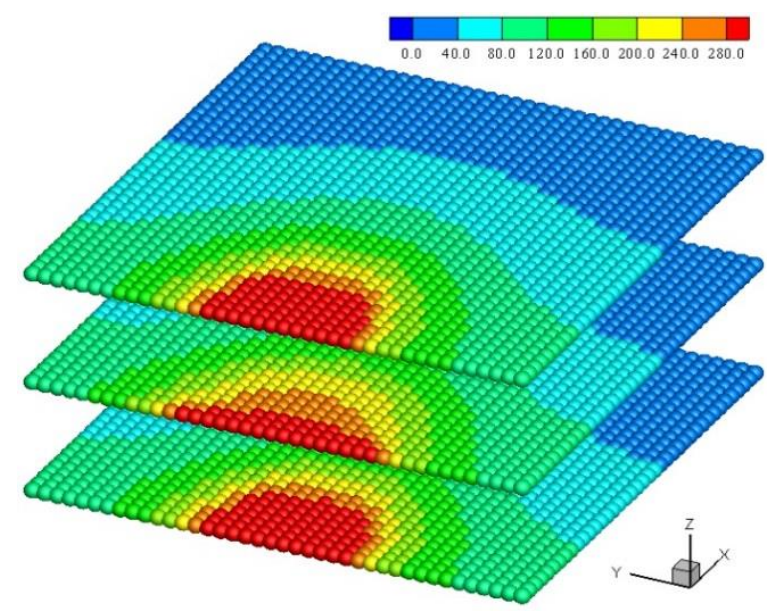

(c) $400 \mathrm{~s}$

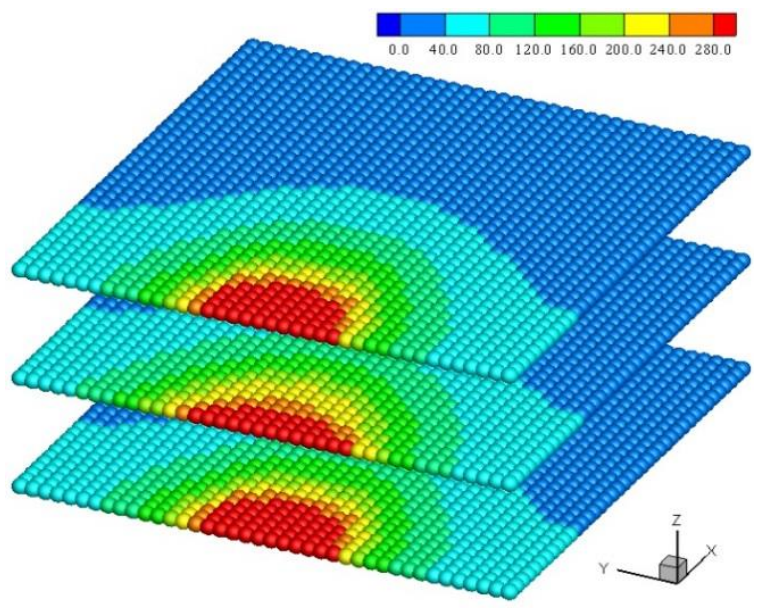

(b) $250 \mathrm{~s}$

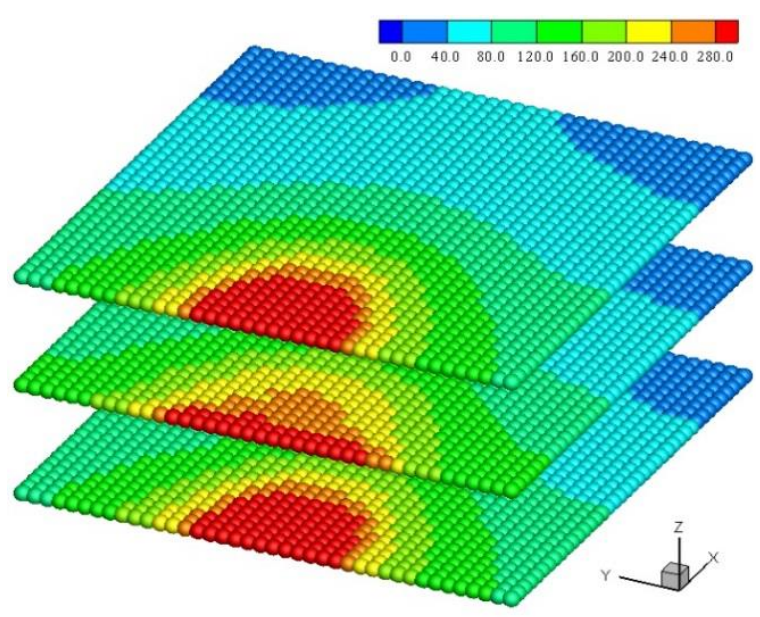

(d) $500 \mathrm{~s}$

Fig. 18 The temperature change distributions at different times

Fig. 19 represents the distribution of $\tilde{T}$ according to Eq.(55a) at $t=500 \mathrm{~s}$. Red colour represents the post glass transition period, the white colour represents the glass transition period, and the blue colour represents the period before the glass transition. The composite points where ignition occurs $\tilde{T}<-2.5$ are removed in the figure. It is observed from the figure that the composite is ignited near the location of the localized fire. 


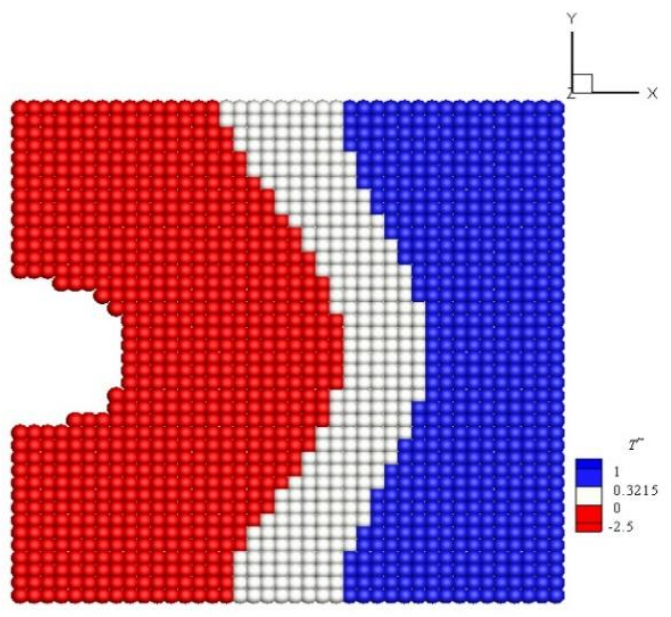

(a) bottom layer

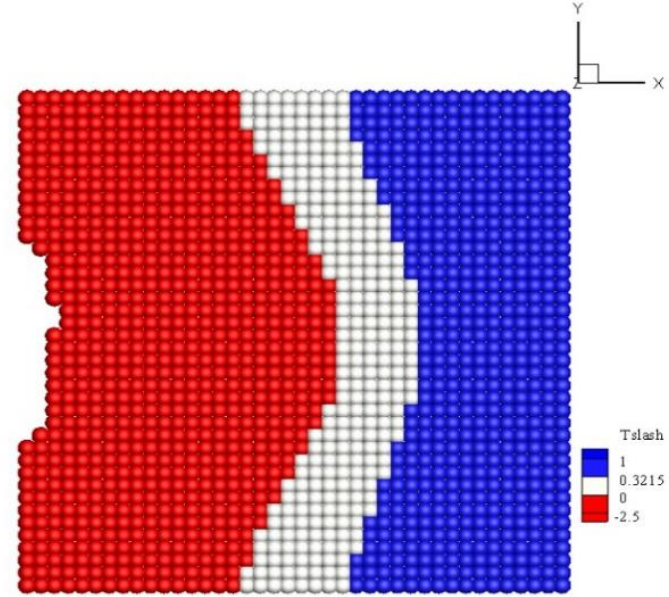

(b) middle layer

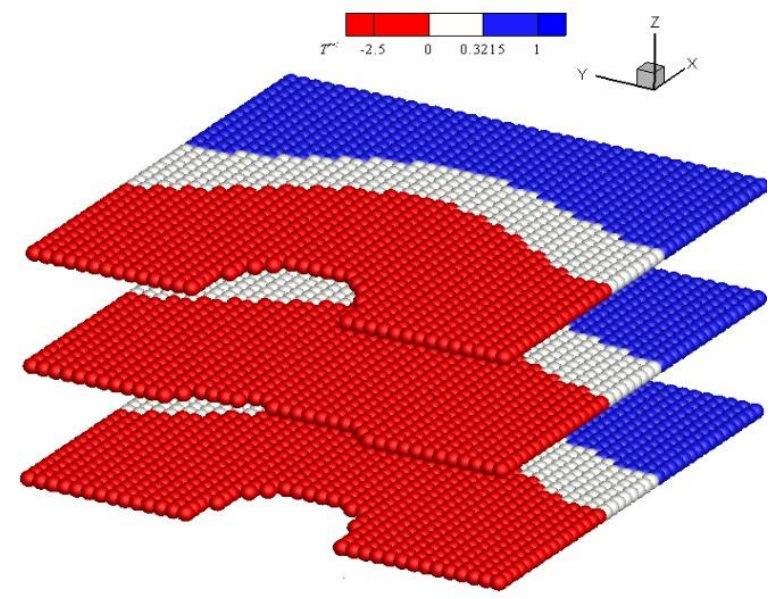

(c) 3D view of all the three layers

Fig. 19 The distribution of $\tilde{T}$ at $t=500 \mathrm{~s}$

The matrix damage, interlayer normal damage, and interlayer shear damage in the mechanical field predicted at different times are provided in Fig. 20 and Fig. 21. During the early stage of the simulation, the crack does not occur until $t=55 \mathrm{~s}$. Then the crack starts to propagate both in the plane and in the thickness direction. 


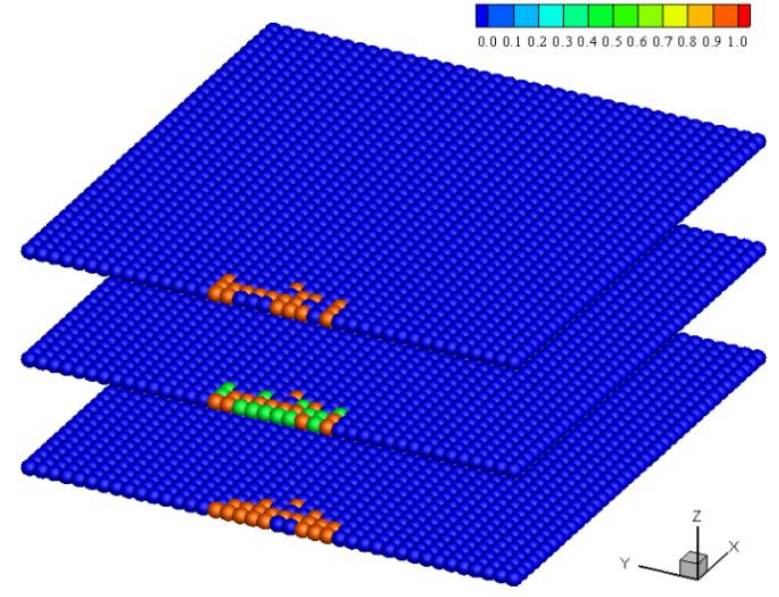

(a) Interlayer normal damage, $\Psi_{i n}$

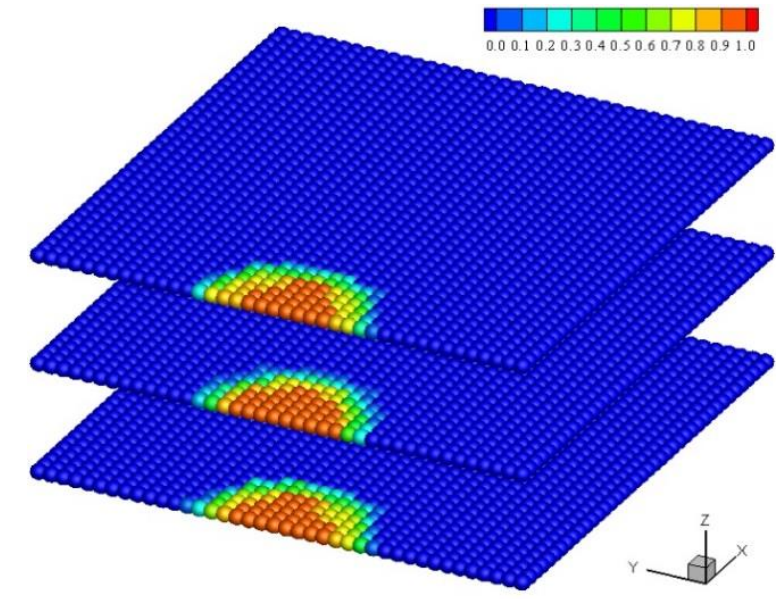

(b) Interlayer shear damage, $\Psi_{\text {is }}$

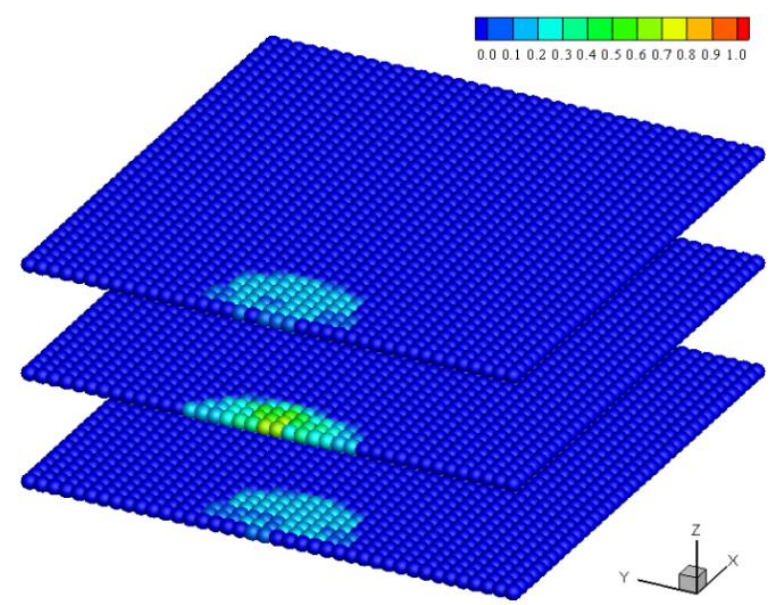

(c) Interlayer matrix damage, $\Psi_{m}$

Fig. 20 Composite mechanical local damage at $250 \mathrm{~s}$ 


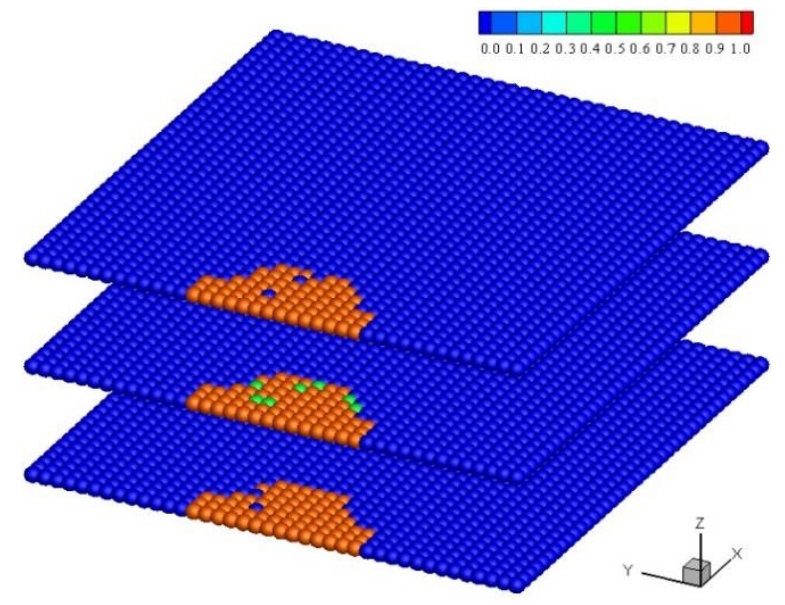

(a) Interlayer normal damage, $\Psi_{\text {in }}$

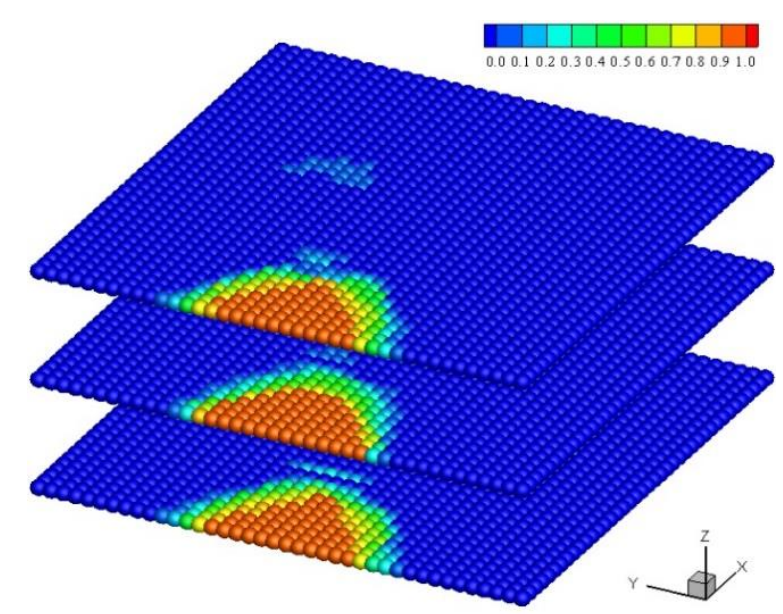

(b) Interlayer shear damage, $\Psi_{\text {is }}$

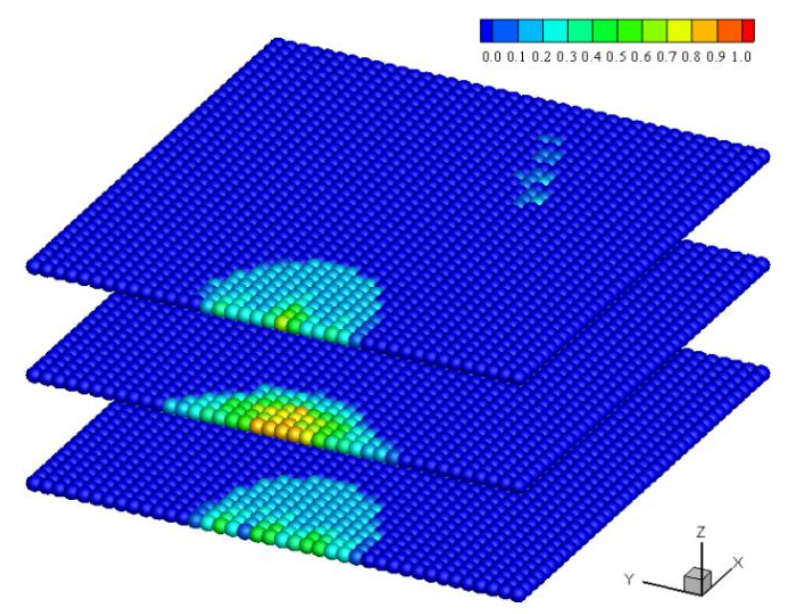

(c) Interlayer matrix damage, $\Psi_{m}$

Fig. 21 Composite mechanical local damage at $500 \mathrm{~s}$

The displacement distributions at $t=100 \mathrm{~s}$ and $t=500 \mathrm{~s}$ are plotted in Fig. 22 and Fig. 23.

Note that, the composite points where the ignition occurs $(\tilde{T}<-2.5)$ and damage parameters $\left(\Psi_{m}, \Psi_{i n}, \Psi_{i s}\right)$ are bigger than 0.5 are removed in both figures. It can be observed from the figures that the region near the local fire damages due to high temperature and mechanical deformation. 


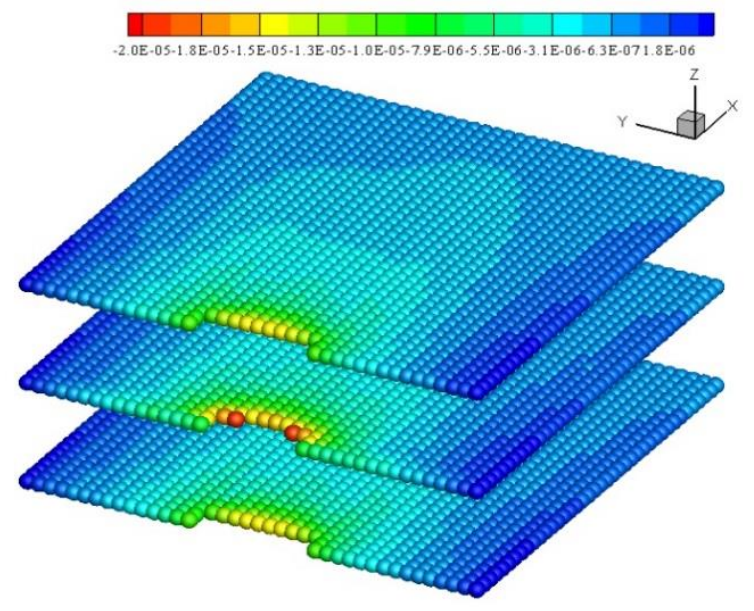

(a)

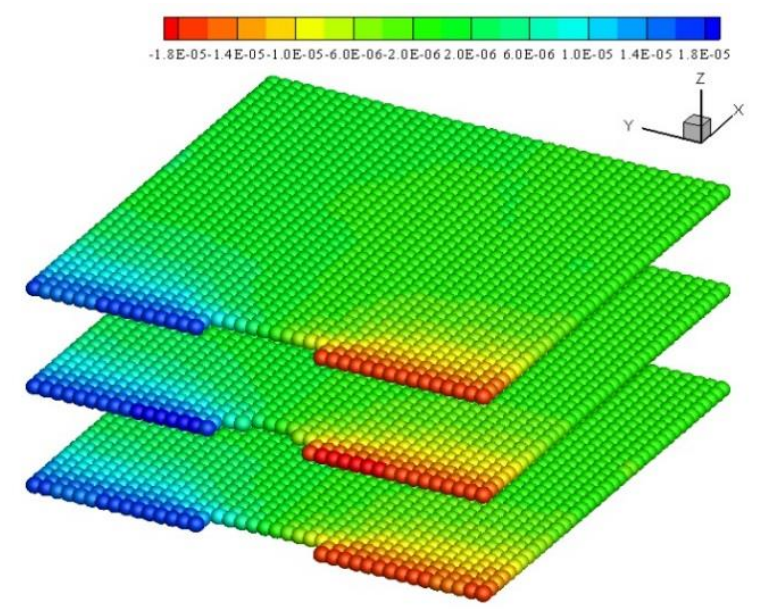

(b)

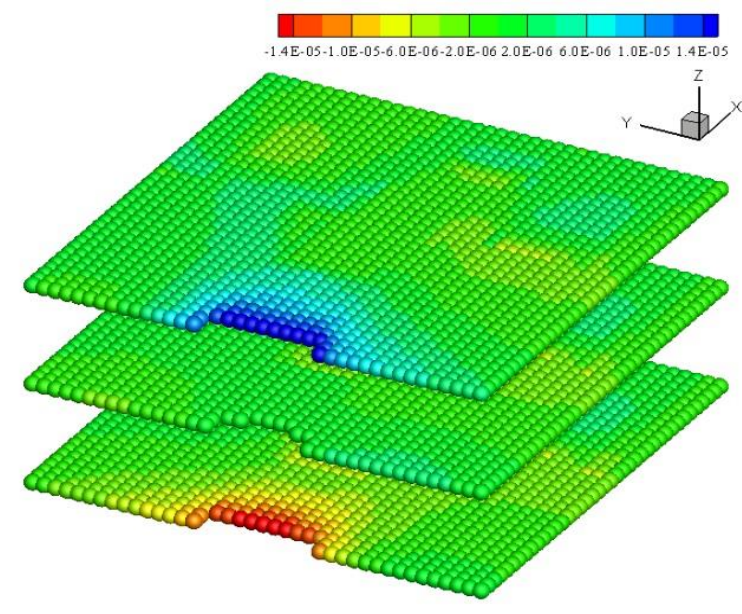

(c)

Fig. 22 Displacement distributions $(\mathrm{m})$ at $t=100 \mathrm{~s}$ : (a) in $x$ direction, (b) in $y$ direction, and (c) in $z$ direction 


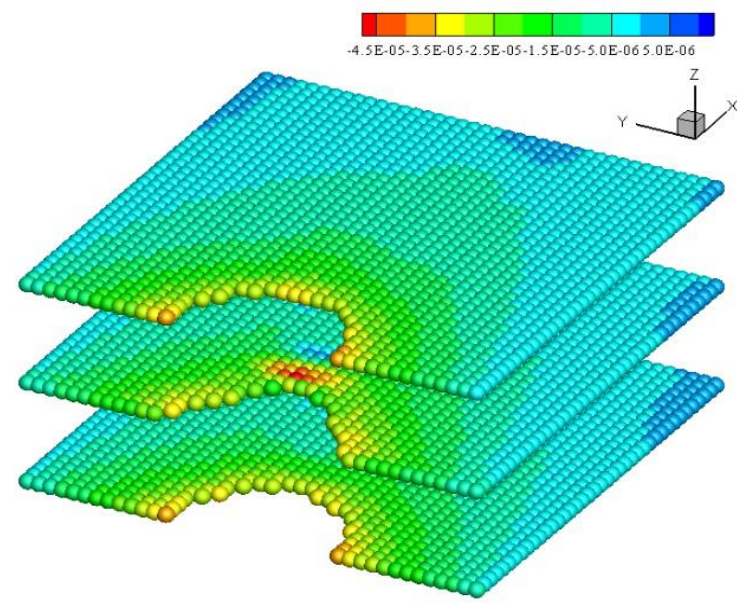

(a)

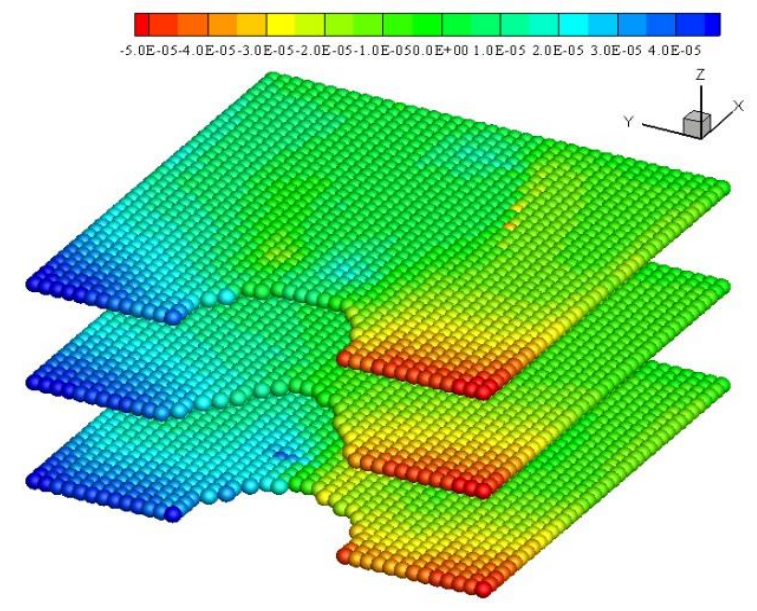

(b)

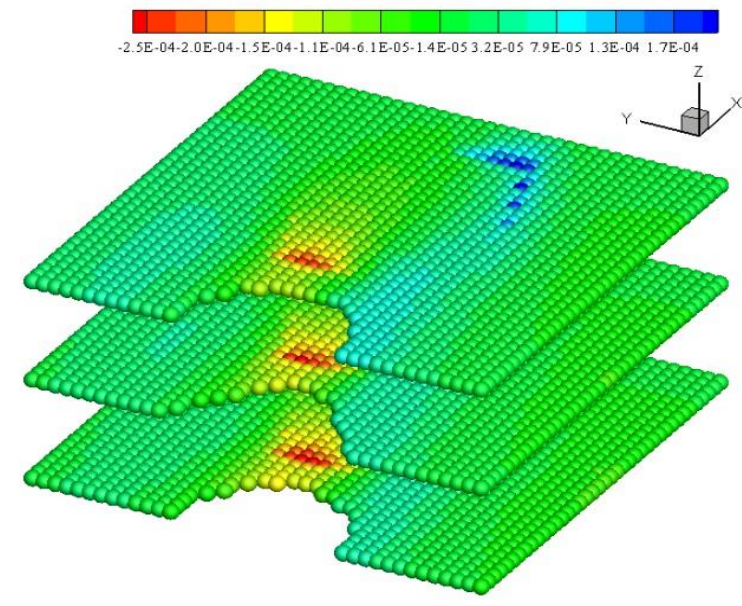

(c)

Fig. 23 Displacement distributions $(\mathrm{m})$ at $t=500 \mathrm{~s}$ : (a) in $x$ direction, (b) in $y$ direction, and (c) in $z$ direction

\subsection{Composite damage due to hot gas induced by fire}

The moving fluid-structure interfaces are one of the typical features in many combustion applications, such as burning and melting surfaces [93]. In this example, the effect of hot gas induced by fire on the composite material is investigated. The hot gas fluid flow and composite model are coupled through the thermal field.

The composite material properties in section 5.1 are used. The temperature profile provided in Eq.(1) is implemented at the boundary to represent a localized fire. The air flow is assumed as two-dimensional. In this section, the one-way coupling approach for the thermal field is used. The heat transfer from composite to air is neglected, only heat transfer from air to composite is considered. In the previous problem, it's found that the mechanical deformations of the 
composite due to temperature change is very small compared to its geometric dimensions. Therefore, the flow field is simulated by considering the initial geometry of the composite. Moreover, the mechanical deformations of the composite due to flow field is also not considered.

\subsubsection{Problem description}

The geometry of the problem is illustrated in Fig. 24. The air (shown in blue) is in a square solid cavity with its non-dimensional dimensions as $L^{*}=W^{*}=1$, and $h_{\text {thick }}^{*}=0.015$. The threelayer composite laminate with the fibre orientation $\left[0 / 90^{\circ} / 0\right]$ is clamped on the left vertical solid boundary (its top layer is illustrated in Fig. 24 by green). The geometry length and width of the laminate are $L_{s}^{*}=W_{s}^{*}=0.5$, and the thickness of each layer is $h_{t h i c k, p l y}^{*}=0.005$. As shown in Fig. 24, the distance between the bottom boundary of the cavity and the bottom of the composite is $d^{*}=0.25$. The length of the local fire region is set as $d_{\text {fire }}^{*}=0.25$.

The horizon size is chosen as $\delta^{*}=3.015 \Delta x^{*}$ for the inplane deformations of the composite model $[47,48,63]$. For the fluid flow model, since the second-order peridynamic differential operator is used, the horizon size is limited to $2 \Delta x \leq \delta \leq 4 \Delta x$ according to [75]. Therefore, the horizon size is also chosen as $\delta^{*}=3.015 \Delta x^{*}$ for the flow field where the point spacing is $\Delta x^{*}=0.0125$. The horizon size for the fluid flow model is also verified as presented in [77, 78]. The total time, $t^{*}=0.25$, and time step size, $\Delta t^{*}=1 \times 10^{-5}$ are chosen for simulating gas flow and heat conduction in composite material.

The non-dimensional parameters are set as $\operatorname{Pr}=0.713$ and $\mathrm{Ra}=10^{3}$ for air. The reference length is set as $L_{0}=0.2 \mathrm{~m}$. The reference velocity is calculated as $v_{0}=\alpha_{f, 0} / L_{0}=10^{-4} \mathrm{~m} / \mathrm{s}$ with the viscous coefficient $\alpha_{f, 0}=2 \times 10^{-5} \mathrm{~m}^{2} / \mathrm{s}$ for air. Therefore, the non-dimensional simulation time $t^{*}=0.25$ corresponds to $500 \mathrm{~s}$. The artificial speed of sound is chosen as $c_{\text {sound }}=10 v_{0}$ [98].

The reference temperature and initial temperature are chosen as $T_{s}^{0}=T_{s, \text { ref }}=293 \mathrm{~K}$, i.e. $T^{*}=0$ for the initial condition. The boundary conditions are also provided in Fig. 24 with grey colour representing the insulated boundary condition, the red one representing the local fire boundary, and the yellow one representing the composite-fluid interface, expressed as 


\section{Thermal field:}

$x^{*}=0$ and $x^{*}=L^{*}$

$$
\frac{\partial T^{*}}{\partial x^{*}}=0
$$

$y^{*}=0$

$$
\begin{aligned}
& x^{*} \leq d_{\text {fire }}^{*}: T^{*}=T_{\text {fire }}^{*}\left(t^{*}\right)=\frac{345 \log \left(8 \times 2000 \times t^{*} / 60+1\right)}{T_{0}} ; 0<t^{*}<0.25 \\
& x^{*}>d_{\text {fire }}^{*}: \frac{\partial T^{*}}{\partial x^{*}}=0
\end{aligned}
$$

$y^{*}=W^{*}$

$$
\frac{\partial T^{*}}{\partial y^{*}}=0
$$

Mechanical field:

$$
x^{*}=0 ; x^{*}=L^{*} ; y^{*}=0 ; y^{*}=W^{*}: \mathbf{u}^{*}=0
$$

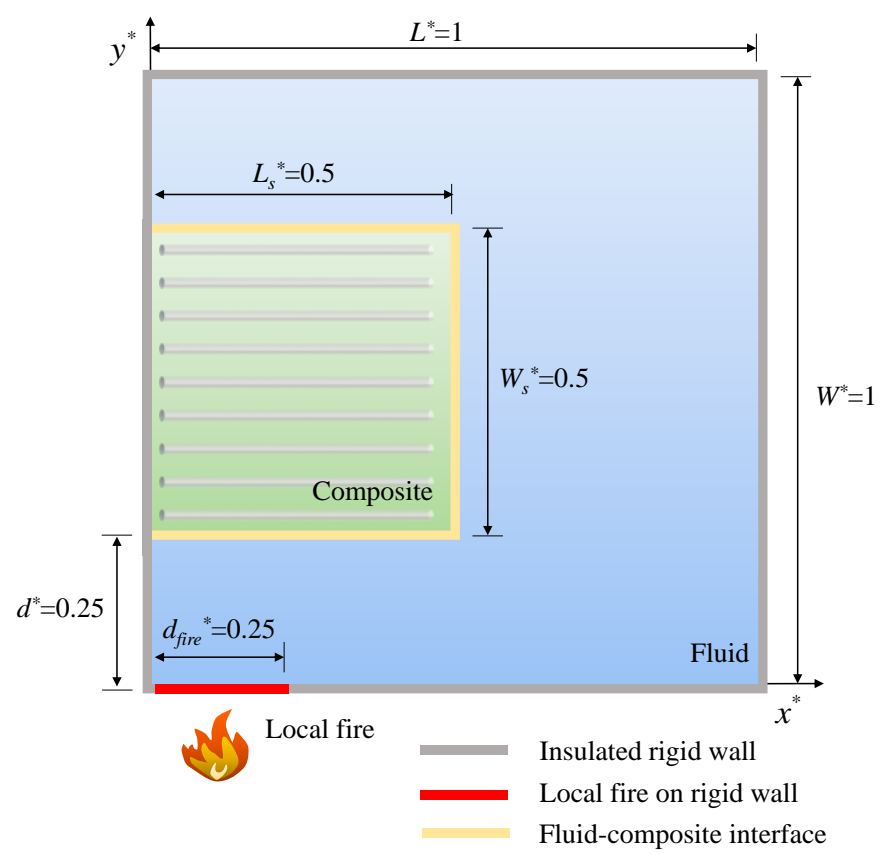

Fig. 24 Geometry illustration for composite and fluid coupling response under the fire scenario 
As observed in Section 5.1, the deformation of composites under fire is relatively small in the in-plane layers compared to its geometry dimensions. Therefore, the composite deformation effect on the fluid field is ignored in the current study for simplicity. For flow simulations, the composite-fluid interface is assumed as a rigid solid wall, as shown in Fig. 25(a).

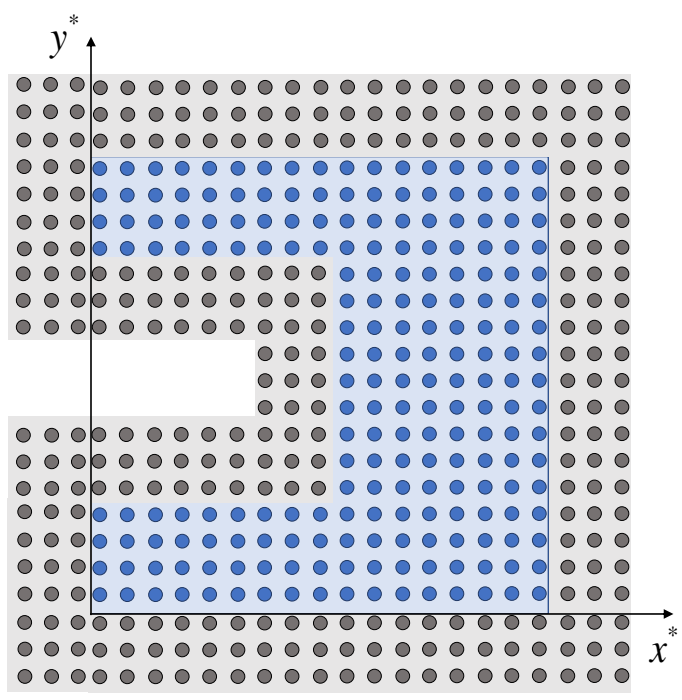

(a)

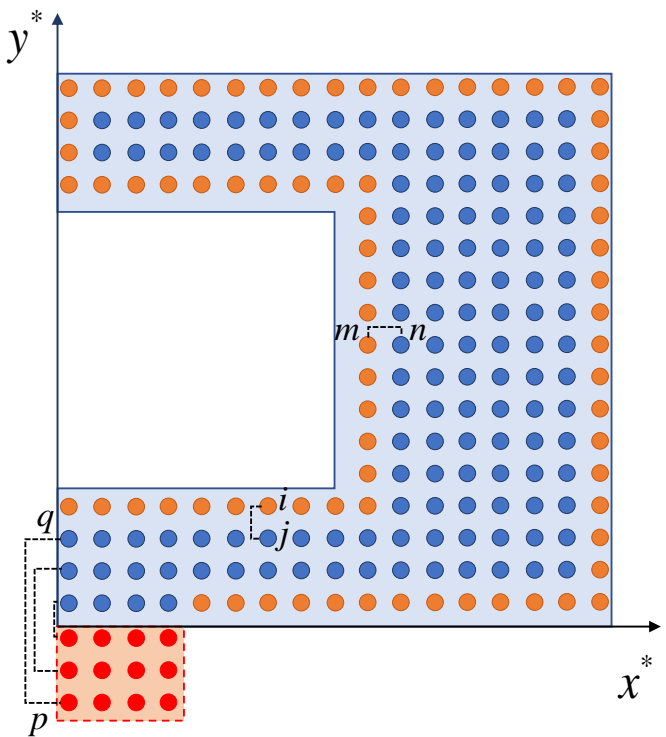

(b)

Fig. 25 PD points for (a) fluid flow field and (b) thermal field

In the mechanical field, all the velocities and displacements of points belonging to the fictitious solid wall layers keep zero throughout the simulation process, i.e. the solid boundary conditions are (grey points in Fig. 25 (a)):

at $x^{*}=L^{*} ; y^{*}=0 ; y^{*}=W^{*} ; x^{*}=0, y^{*}<d^{*} ; x^{*}=0, y^{*}>W^{*}-d^{*} ; x^{*}=L_{s}^{*}, d^{*} \leq y^{*} \leq W^{*}-d^{*} ;$ $0<x^{*} \leq L_{s}^{*}, y^{*}=d^{*}$ and $0<x^{*} \leq L_{s}^{*}, y^{*}=W^{*}-d^{*}$ :

$$
\mathbf{u}_{f}^{*}=0
$$

In the fluid thermal field, the thermal boundary conditions are

At $x^{*}=0, y^{*}<d^{*}, ; x^{*}=0, y^{*}>W^{*}-d^{*} ; x^{*}=L_{s}^{*}, d^{*} \leq y^{*} \leq W^{*}-d^{*}$ and $x^{*}=L^{*}$ (orange points in Fig. 25 (b)):

$$
\frac{\partial T_{f}^{*}}{\partial x^{*}}=0: T_{f, m}^{*}=T_{f, n}^{*} \text { for pairwise points }
$$

At $0<x^{*} \leq L_{s}^{*}, y^{*}=d^{*} ; 0<x^{*} \leq L_{s}^{*}, y^{*}=W^{*}-d^{*} ; y^{*}=W^{*} ; y^{*}=0, x^{*} \geq d_{\text {fire }}^{*}$ and $x^{*}=L^{*}$ (orange points in Fig. 25 (b)): 


$$
\frac{\partial T_{f}^{*}}{\partial y^{*}}=0: T_{f, i}^{*}=T_{f, j}^{*} \text { for pairwise points }
$$

At $y^{*}=0, x^{*}<d_{\text {fire }}^{*}($ for red points in Fig. $25(\mathrm{~b}))$ :

$$
T_{f, p}^{*}=2 T_{\text {fire }}^{*}\left(t^{*}\right)-T_{f, q}^{*}
$$

The composite material is the same as the one in Section 5.1. Regarding the composite PD model, the composite points' initial spacing is the same as the fluid spacing, i.e. $\Delta x^{*}=0.0125$ with $40 \times 40 \times 3$ points. The location $z=0$ is in the midplane of the composite model.

The front view of the composite model for the mechanical field and thermal field is provided in Fig. 26, respectively. In Fig. 26 (a), the fictitious layers on the left represent the vertical solid wall, and the green points represent the composite laminate. In Fig. 26 (b), no fictitious layer is added in the thermal field. The orange points in Fig. 26 (b) are under insulated-boundary conditions while the purple points are under the convection and radiation condition related to the fluid flow.

Thermal boundary conditions are:

At $x^{*}=0$

$$
\frac{\partial T_{s}^{*}}{\partial x^{*}}=0: T_{s, i}^{*}=T_{s, j}^{*} \text { for pairwaise points }
$$

At $x^{*}=L_{s}^{*}, y^{*}=d^{*}$, and $y^{*}=W^{*}-d^{*}$

$$
\begin{aligned}
h_{s}^{*}\left(\mathbf{x}_{s, \text { interface }}^{*}, t^{*}\right)= & -\frac{1}{\Delta x^{*}} h_{\text {con }}^{*}\left(T_{s}^{*}\left(\mathbf{x}_{s, \text { interface }}^{*}, t^{*}\right)-T_{f, \text { local }}^{*}\right) \\
& +\sigma_{\text {stefan-boltzmann }}^{*} \varepsilon \frac{1}{\Delta x^{*}}\left(\left(T_{f, \text { local }}^{*}\right)^{4}-\left(T_{s}\left(\mathbf{x}_{s, \text { interface }}^{*}, t^{*}\right)\right)^{4}\right)
\end{aligned}
$$

Mechanical boundary conditions:

At $x^{*}=0$

$$
\mathbf{u}_{s}^{*}=0
$$




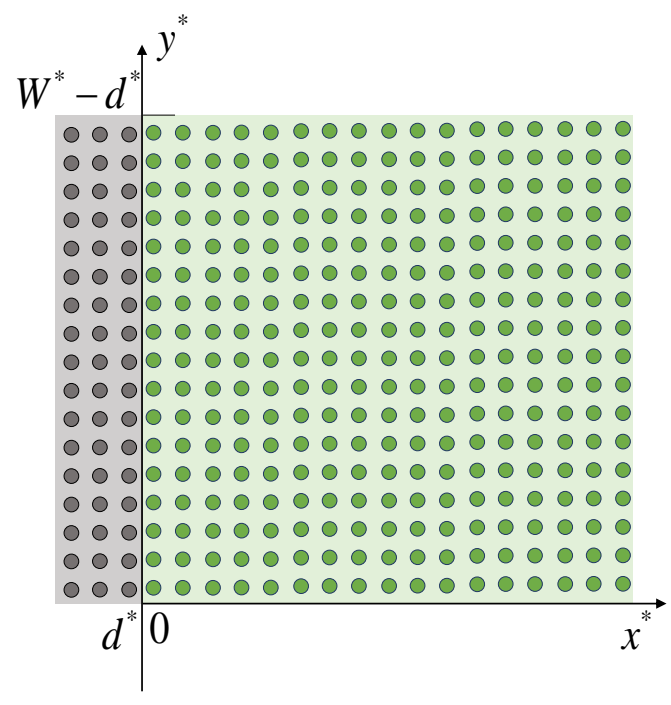

(a)

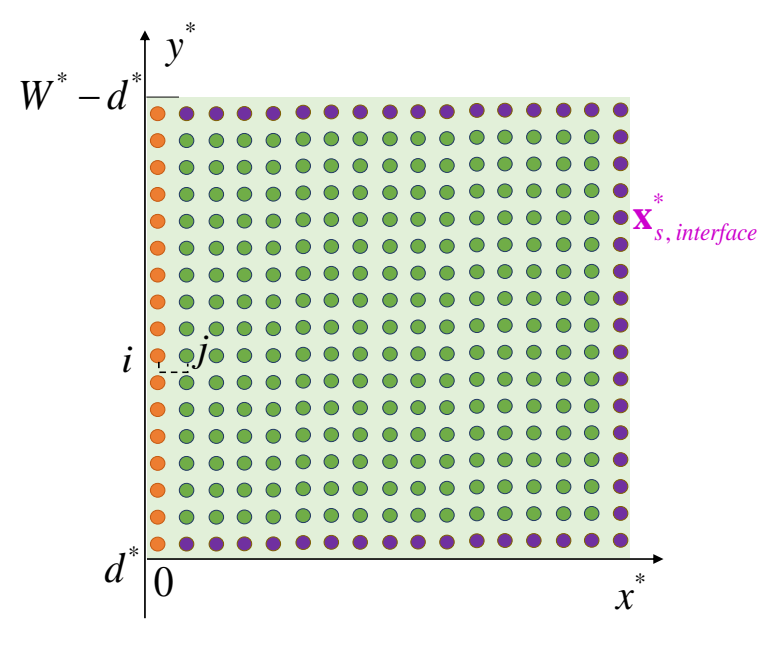

(b)

Fig. 26 PD material points for (a) composite mechanical field,

(b) composite thermal field

\subsubsection{Numerical procedure}

The flowchart for problem 5.2 is provided in Fig. 27. First, the thermo-fluid flow is simulated in a non-dimensional form under the fire boundary condition. Thus, the dimensionless temperature profiles of the fluid are predicted. Subsequently, the heat is transferred from the fluid field to the composite through the convection and radiation interface boundaries. The dimensionless temperature change in the composite is predicted by solving the composite heat conduction equation under the interface boundary conditions. Then the dimensionless temperature of the composite is converted into its dimensional value, in order to update the composite material properties both including the thermal properties and the mechanical properties. This procedure, i.e. thermo-fluid flow and composite heat conduction, is performed every time step as a transient analysis. Subsequently, being the same as Section 5.1, the deformation of the PD composite model due to temperature change is predicted by using the ADR technique [97] for each 100 seconds as a quasi-static analysis. The composite mechanical analysis is performed in a dimensional form by using the converted dimensional temperature profiles. Besides, it should be noted that the non-dimensional values of the composite geometry are used in the composite mechanical analysis, which can be treated as a geometry scaled composite model. Therefore, the predicted deformation such as displacement is also scaled. 


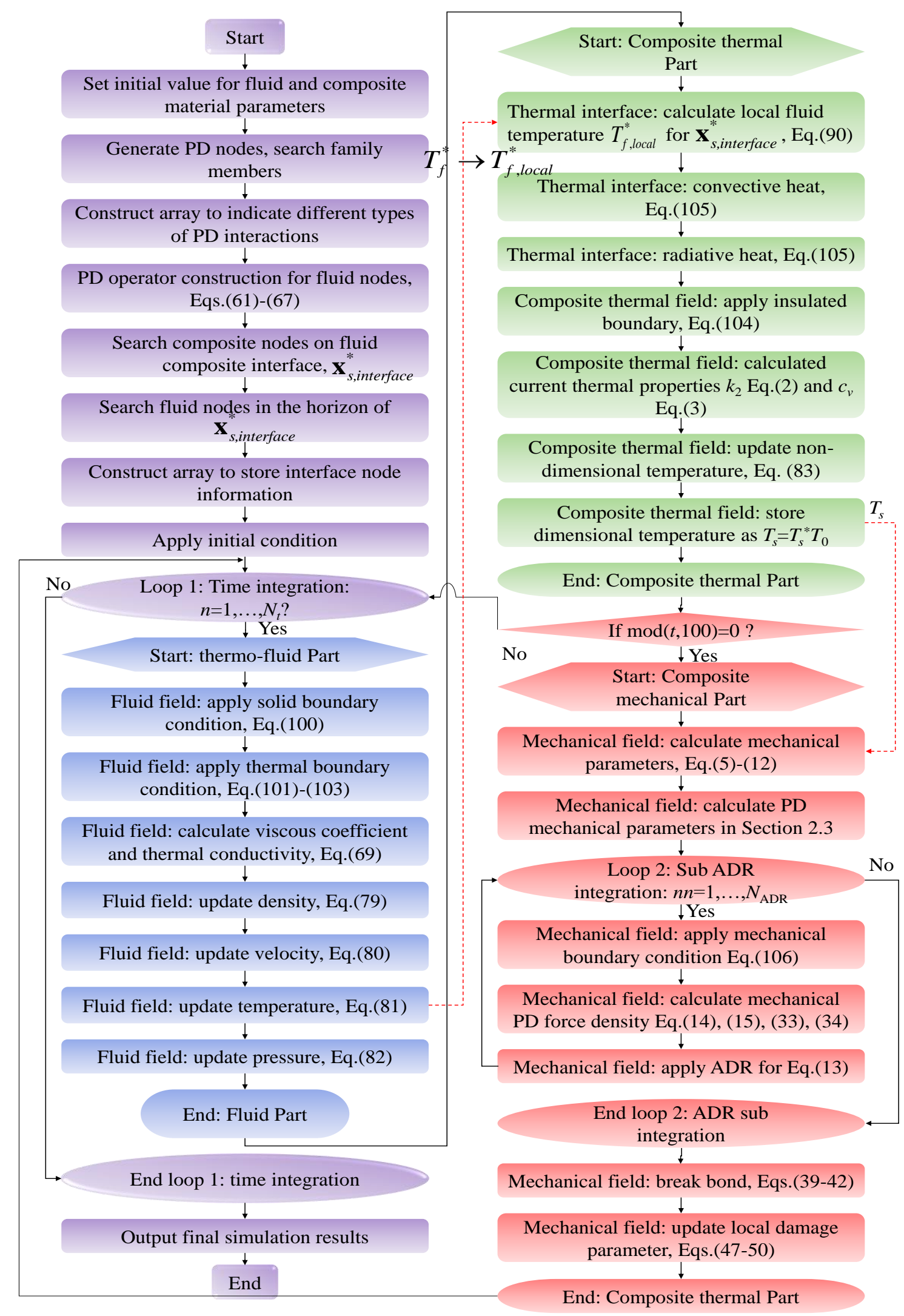

Fig. 27 Flowchart for the coupled thermo-fluid model for air and thermo-mechanical model for composite for problem 5.2 


\subsubsection{Numerical results}

The temperature profiles in fluid with respect to the reference temperature, $\left(T_{f}-T_{r e f}\right)$ are provided in Fig. 28. In order to be comparable to the fire temperature-time curve, the dimensional temperatures at different times are provided. It can be observed from Fig. 28 that the interface between the composite bottom edge and fluid suffers the high temperature while the temperatures around other interfaces are relatively low.

Non-dimensional velocity profiles of fluid at different times are provided in Fig. 29. The fluid flows due to the temperature difference between boundaries. The largest velocity occurs just below the composite bottom edge at $t^{*}=0.05$. Then the air will flow up due to the temperature difference between the high temperature on the bottom and the relatively low temperature on the top. In this example $\mathrm{Ra}=10^{3}$ is used and it is much smaller than $\mathrm{Ra}=10^{6}$ for turbulent flow [99], therefore the fluid flow shown in Fig. 29 is laminar. The fluid field in problem 5.2 is compared with [93] qualitatively. Both in the present study and [93], the region between the fire boundary and the bottom edge of composite experiences the highest temperature. Similar flow pattern is also observed in [93] for the similar flow geometry and fire location, where the region between the fire boundary and the bottom edge of composite and the region between the right vertical edge of composite and the right vertical edge of the container experience the largest velocity. 


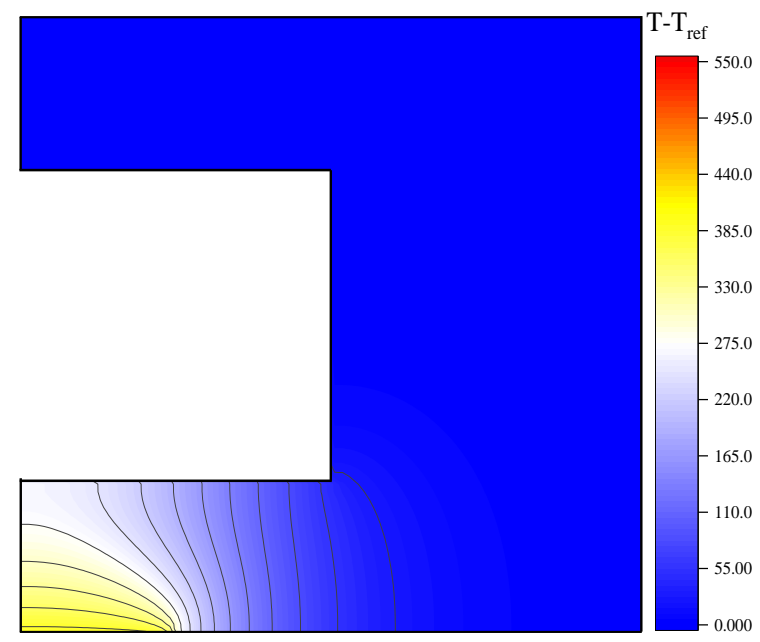

(a)

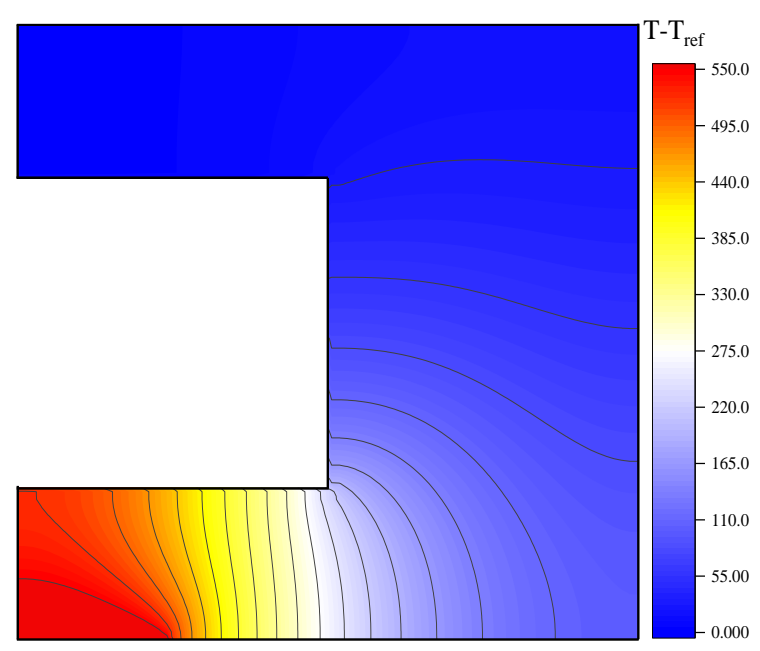

(c)

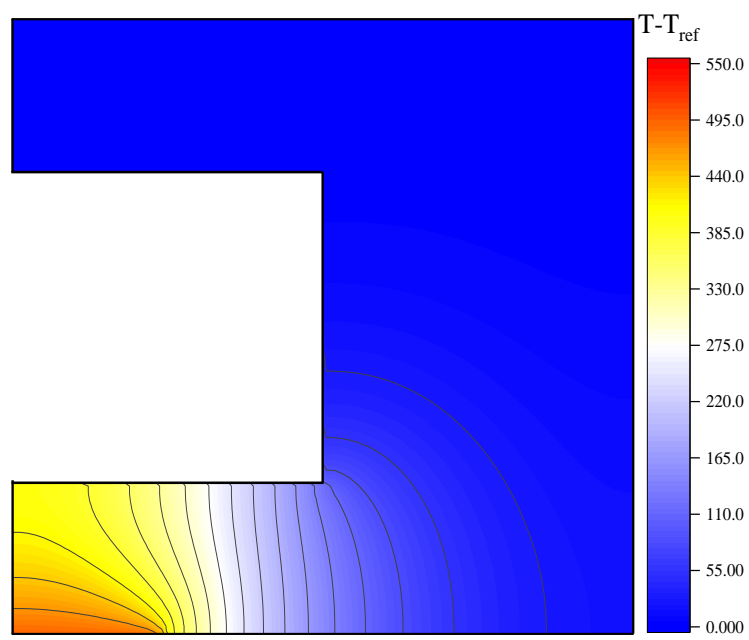

(b)

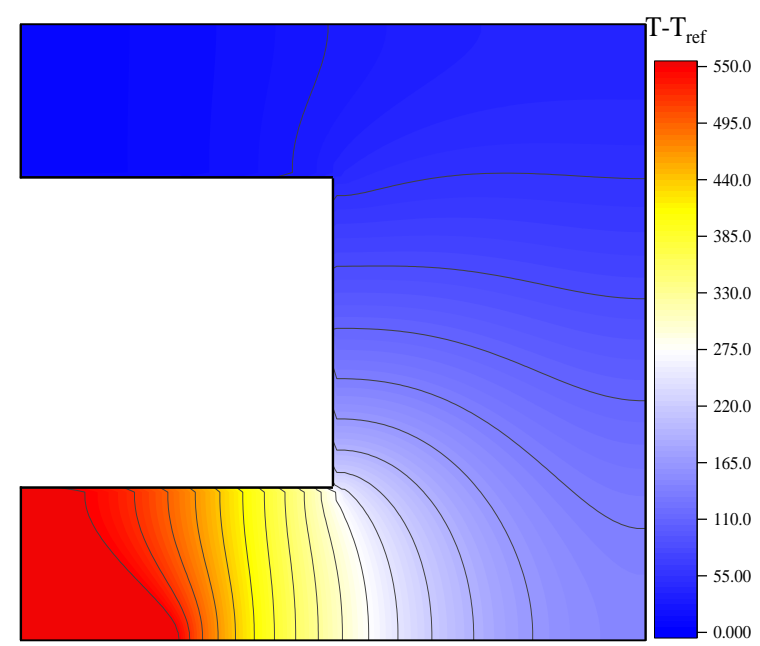

(d)

Fig. 28 Temperature profiles in fluid with respect to reference temperature, . . $\left(T_{f}-T_{\text {ref }}\right)$ at time (a) $t=100 \mathrm{~s}\left(t^{*}=0.05\right)$, (b) $t=200 \mathrm{~s}\left(t^{*}=0.1\right)$, (c) $t=400 \mathrm{~s}\left(t^{*}=0.2\right)$, and (d)

$$
t=500 \mathrm{~s}\left(t^{*}=0.25\right)
$$




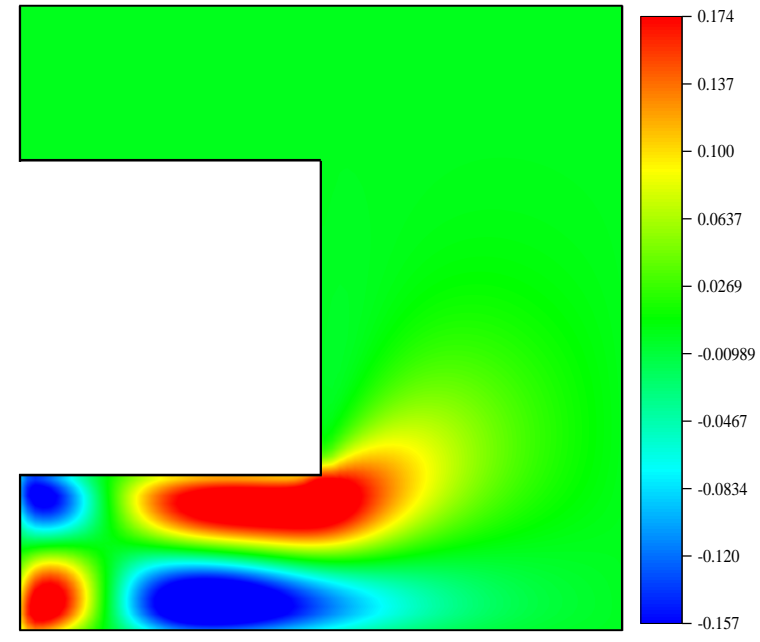

(a) $v_{x}^{*}$, at $t^{*}=0.05$

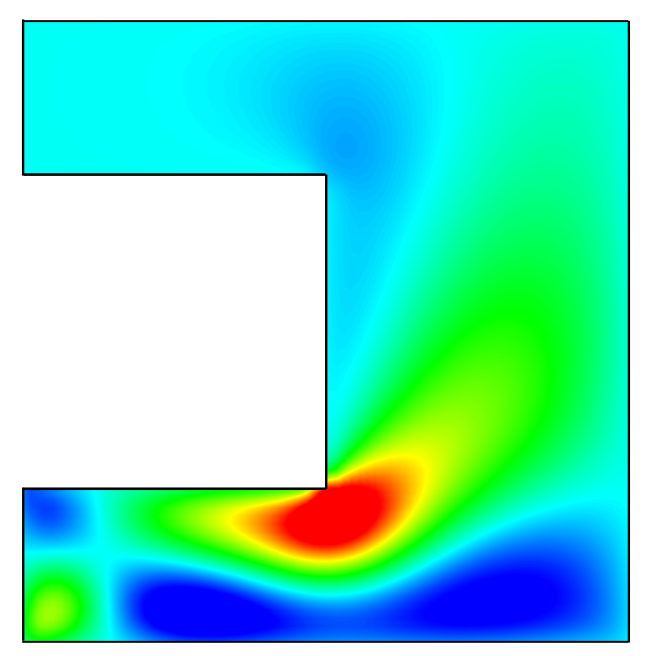

(c) $v_{x}^{*}$, at $t^{*}=0.1$

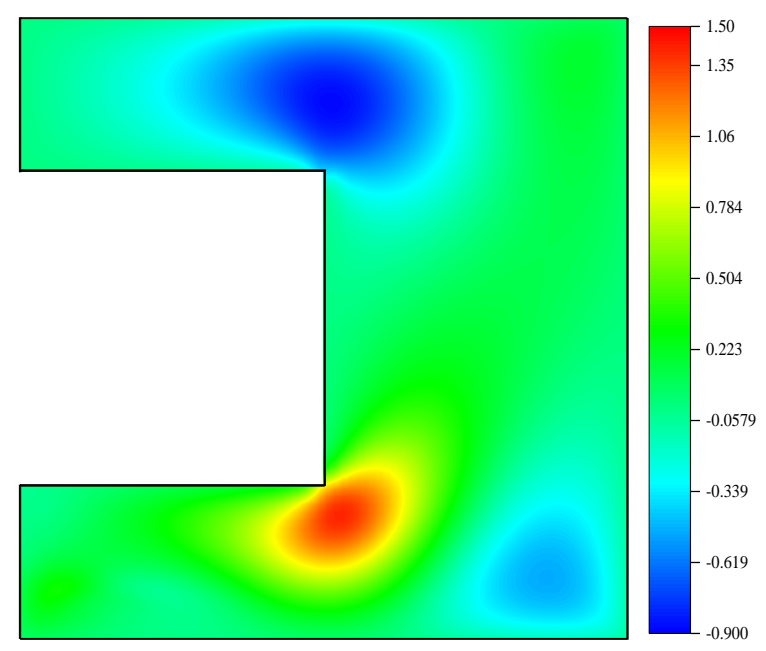

(e) $v_{x}^{*}$, at $t^{*}=0.2$

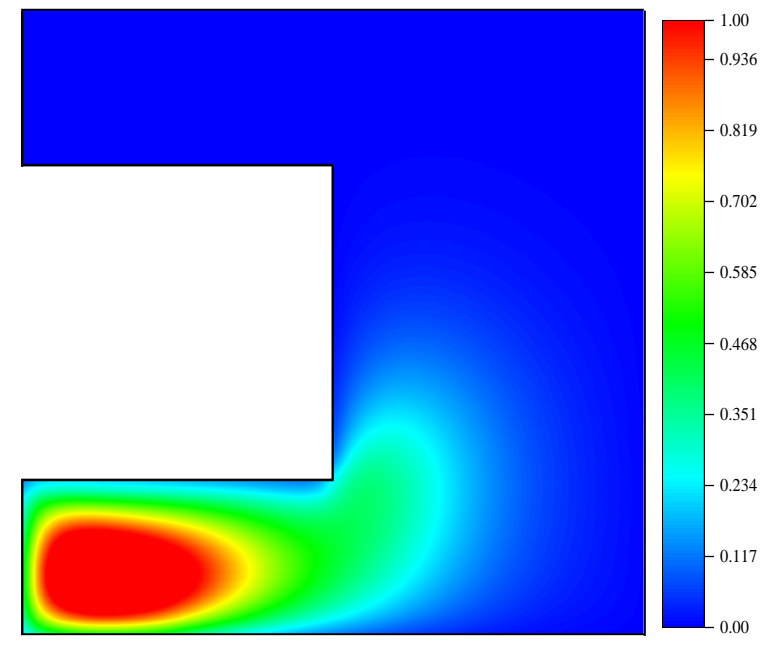

(b) $v_{y}^{*}$, at $t^{*}=0.05$

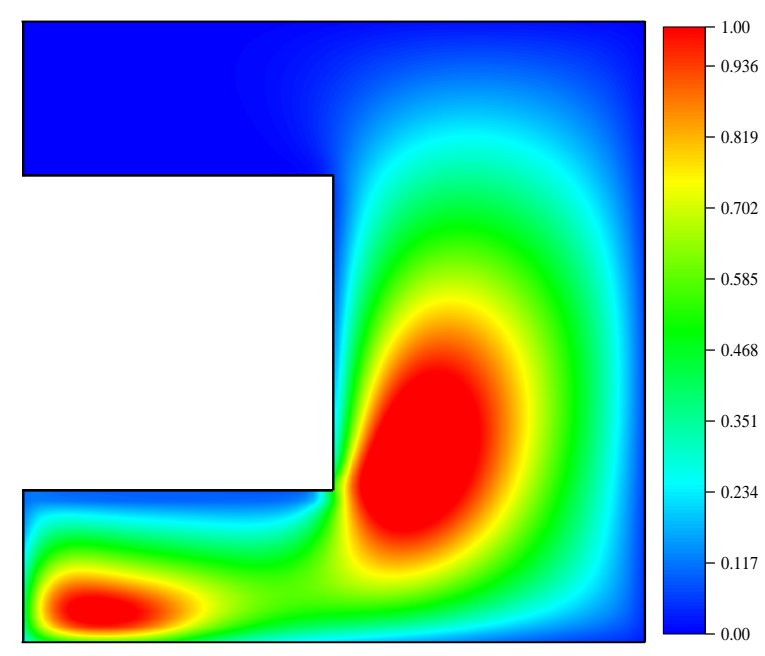

(d) $v_{y}^{*}$, at $t^{*}=0.1$

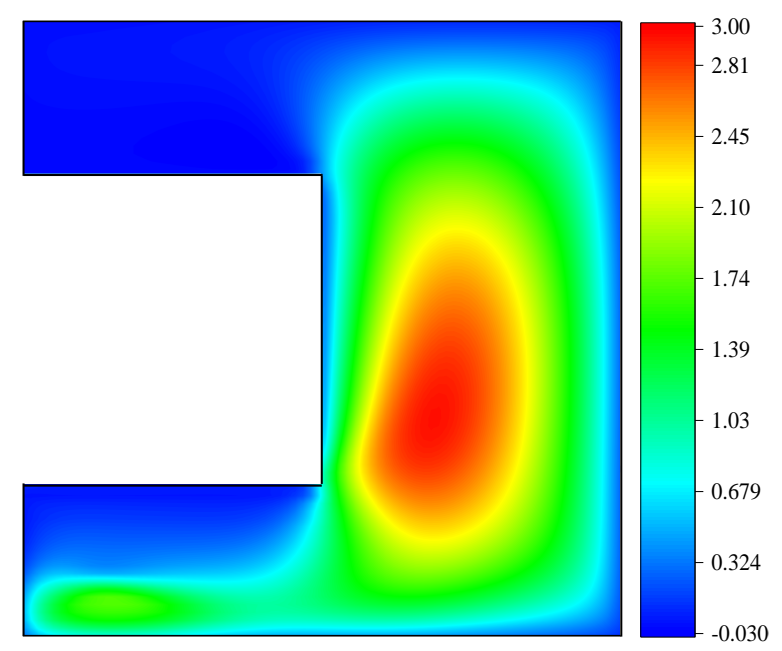

(f) $v_{y}^{*}$, at $t^{*}=0.2$ 


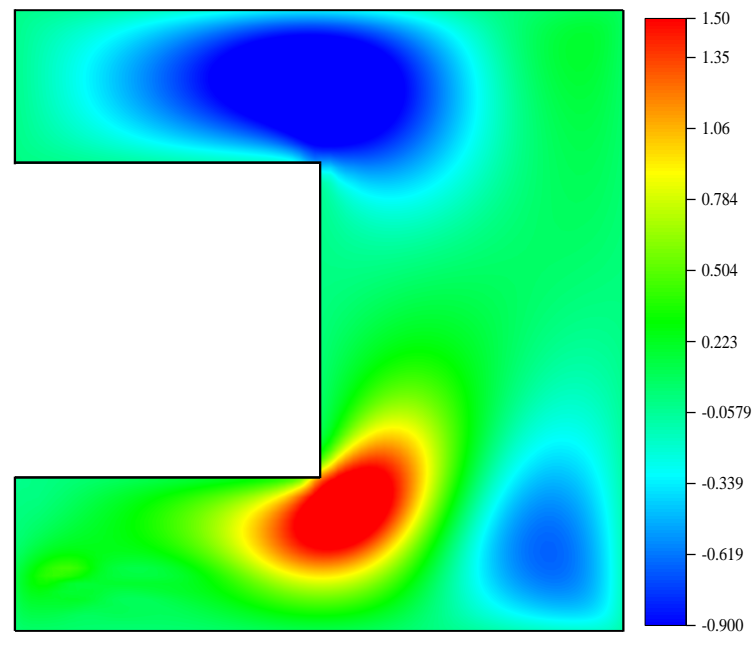

(g) $v_{x}^{*}$, at $t^{*}=0.25$

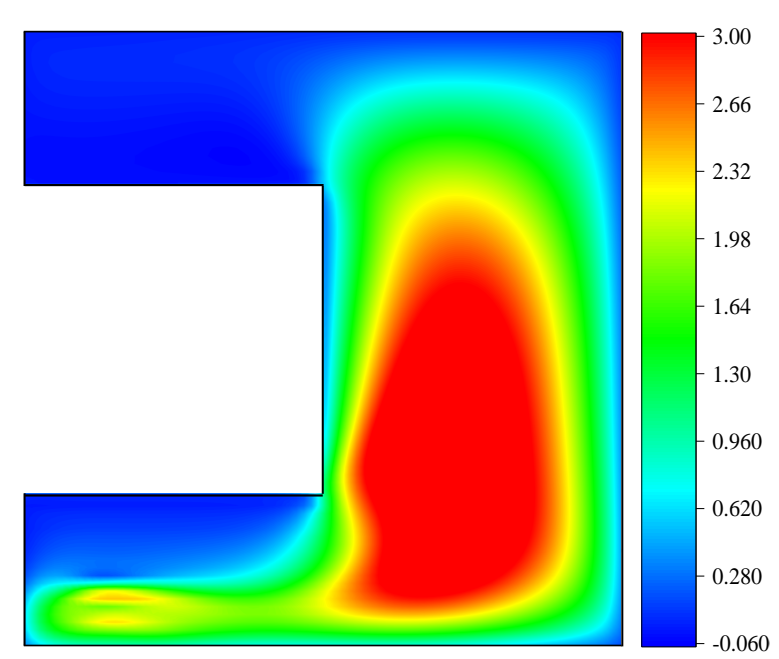

(h) $v_{y}^{*}$, at $t^{*}=0.25$

Fig. 29 Non-dimensional velocity profiles of fluid at different times

Regarding the thermal field of the composite laminate, the temperature profiles in composites with respect to the reference temperature are provided in Fig. 30 at different times. It can be observed from Fig. 30 that due to the anisotropic property and different stacking sequence of the laminates, the temperature distributions are different for different layers at the same time. The rate of temperature rise is relatively small due to the small local fired region and the low thermal conductivity of composite materials used in the present study. According to the investigation performed in [81], the temperature rising rate of the average temperature of the same composite material is only $0.07 \mathrm{~K} / \mathrm{s}$ [81]. In this example, the rising rate is 0.085 $\mathrm{K} / \mathrm{s}$ for the bottom layer and $0.061 \mathrm{~K} / \mathrm{s}$ for the middle layer, which is consistent with the investigation in [81]. In the previous example in Section 5.1, the temperature rising rate of the average temperature of laminates is $0.21 \mathrm{~K} / \mathrm{s}$. Compared to the previous problem in which the composite is directly imposed under the fire-induced temperature boundary condition, the temperature rise in this simulation case is much smaller. Furthermore, no damage due to ignition of composite material is observed. 


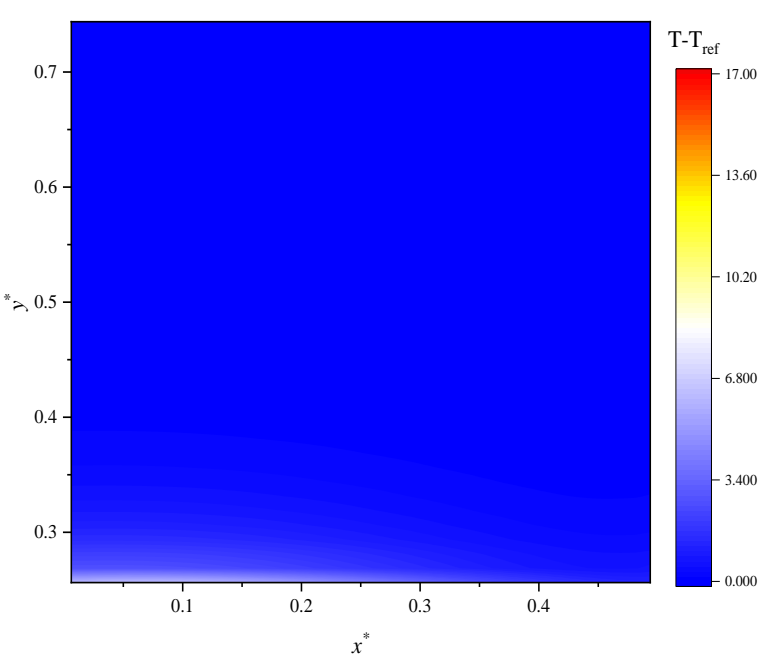

(a) bottom layer, at $t=100 \mathrm{~s}$

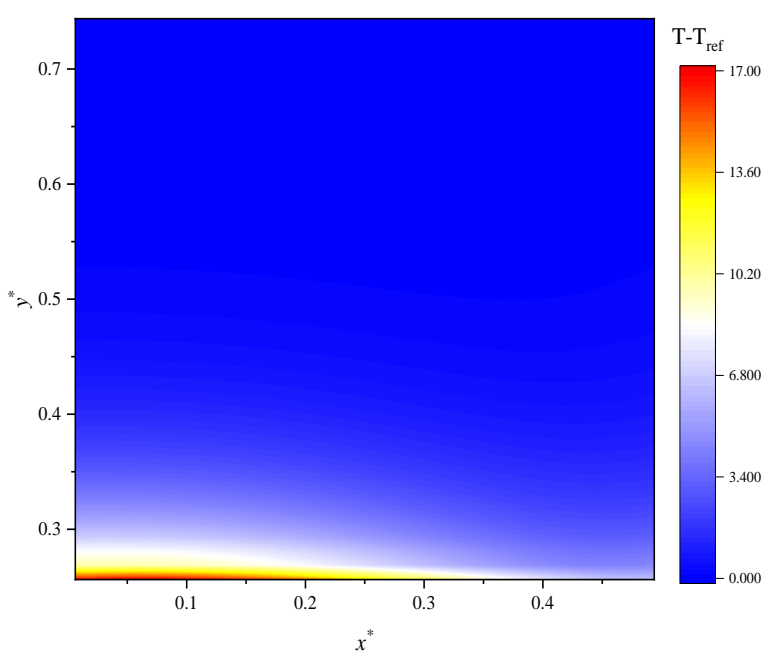

(c) bottom layer, at $t=200 \mathrm{~s}$

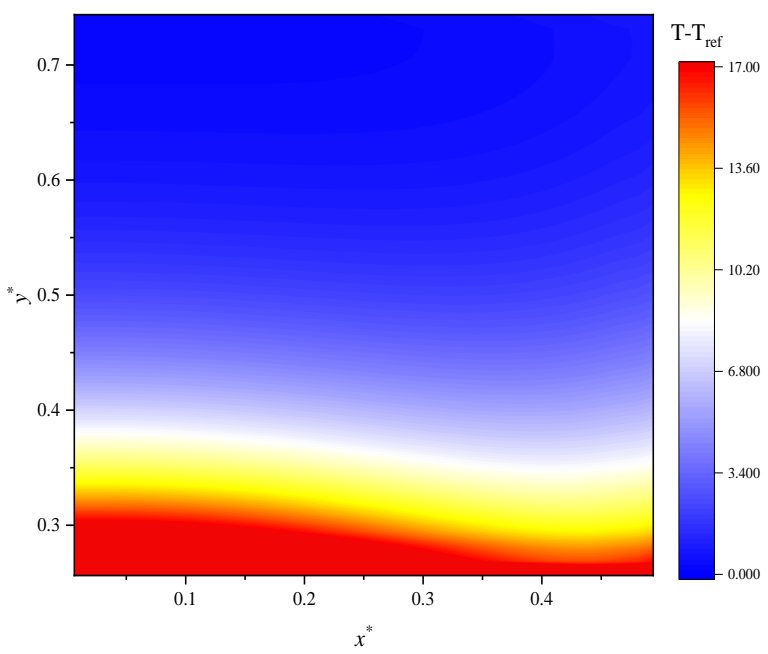

(e) bottom layer, at $t=400 \mathrm{~s}$

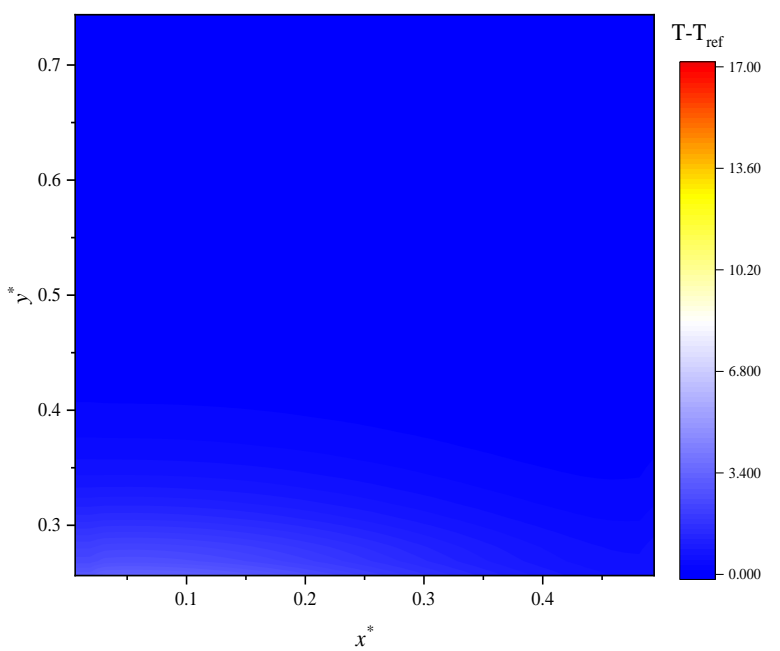

(b) middle layer, at $t=100 \mathrm{~s}$

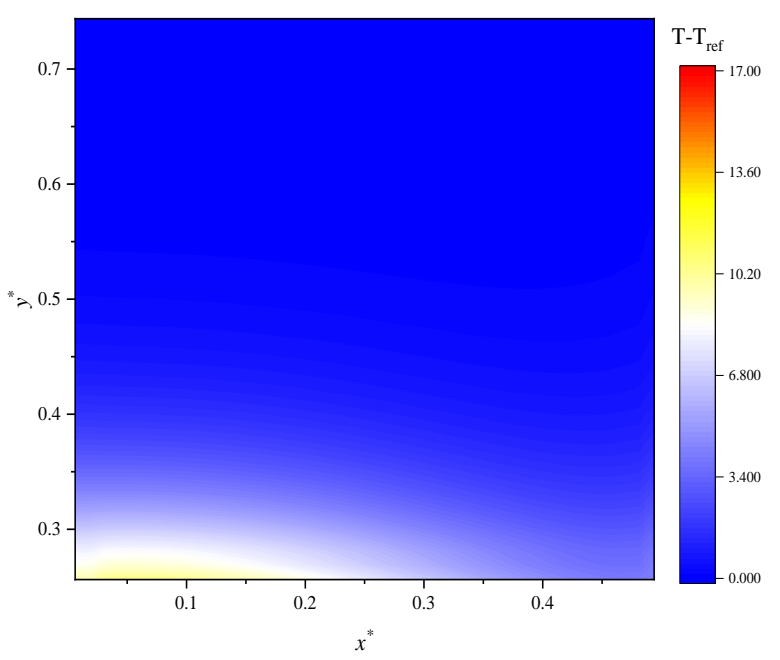

(d) middle layer, at $t=200 \mathrm{~s}$

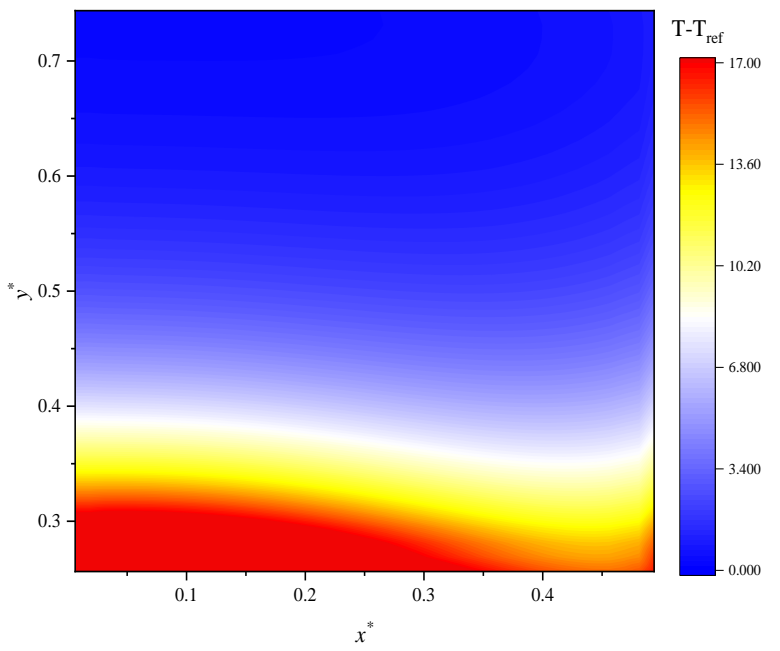

(f) middle layer, at $t=400 \mathrm{~s}$ 


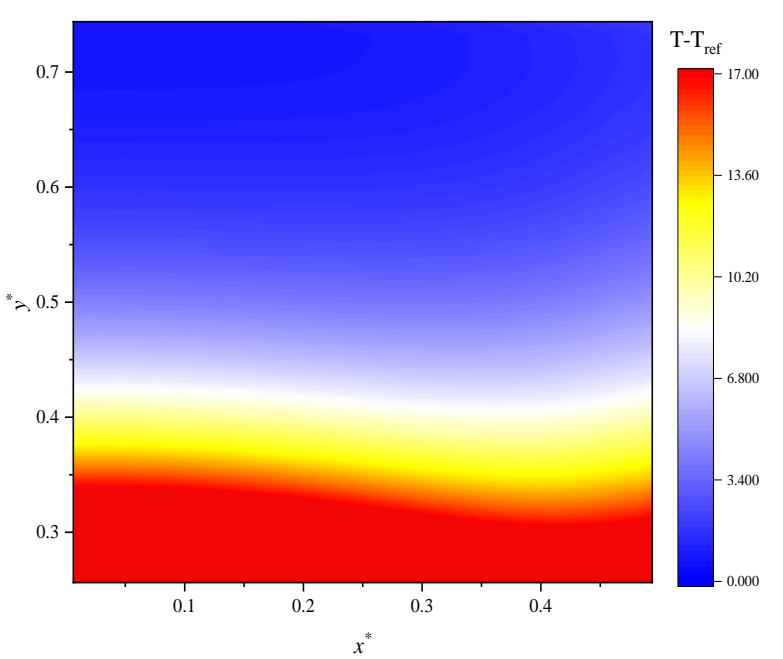

(g) bottom layer, at $t=500 \mathrm{~s}$

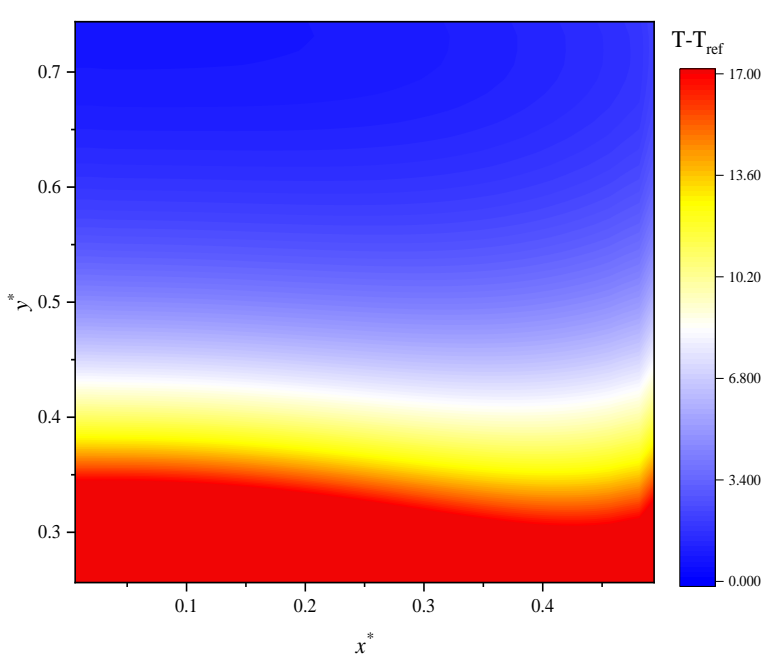

(h) middle layer, at $t=500 \mathrm{~s}$

Fig. 30 Temperature profiles in composite with respect to the reference temperature, $T_{r e f}$

$$
\left(T_{s}-T_{r e f}\right) \text { at different times }
$$

The deformation of the composite is predicted for each 100 seconds by using the ADR approach [97]. The total number of iterations in one ADR performance is $N_{A D R}=4000$. Fig. 31 represents the convergence of displacement fields in ADR [97] simulations at time $t^{*}=0.25$. The non-dimensional displacements of the three-layer composite at different times are provided in Fig. 32-Fig. 33. The deformed shape at the final stage is provided in Fig. 34. It can be observed from the figure that the delamination tends to occur in the heated region because of the thermal expansion.

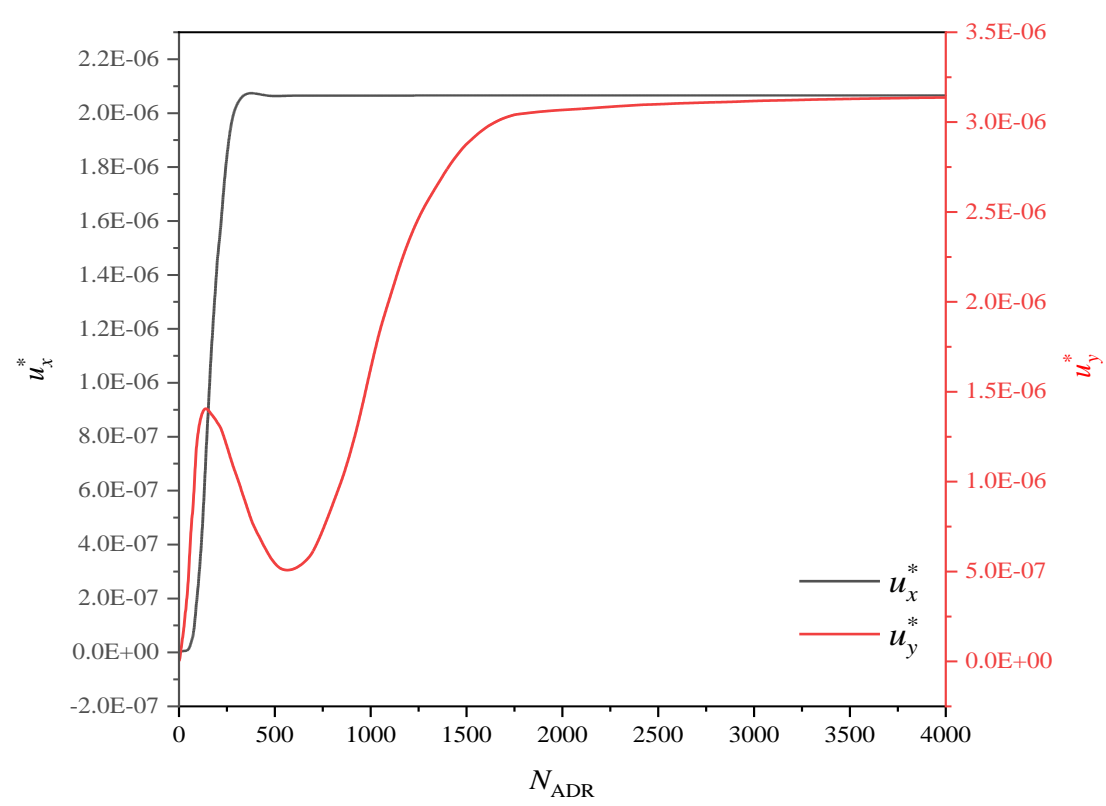

Fig. 31 Convergence of non-dimensional displacement fields for ADR [97] at $t^{*}=0.25$ 


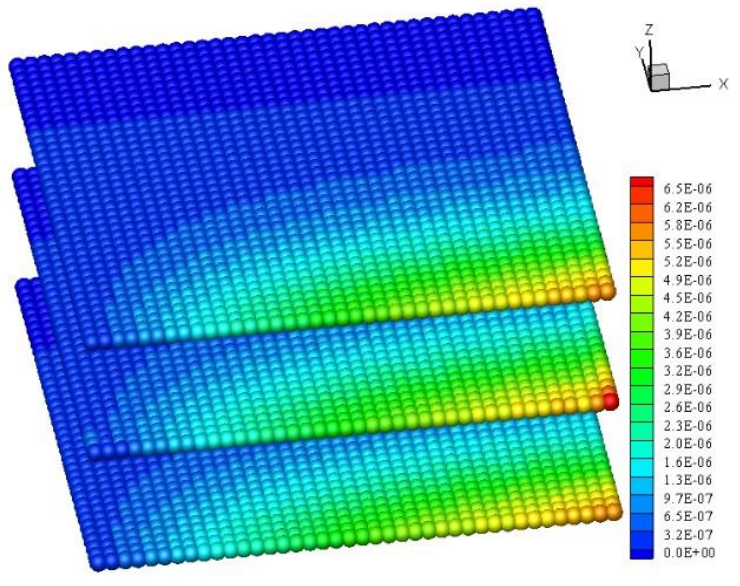

(a) $u_{x}^{*}$

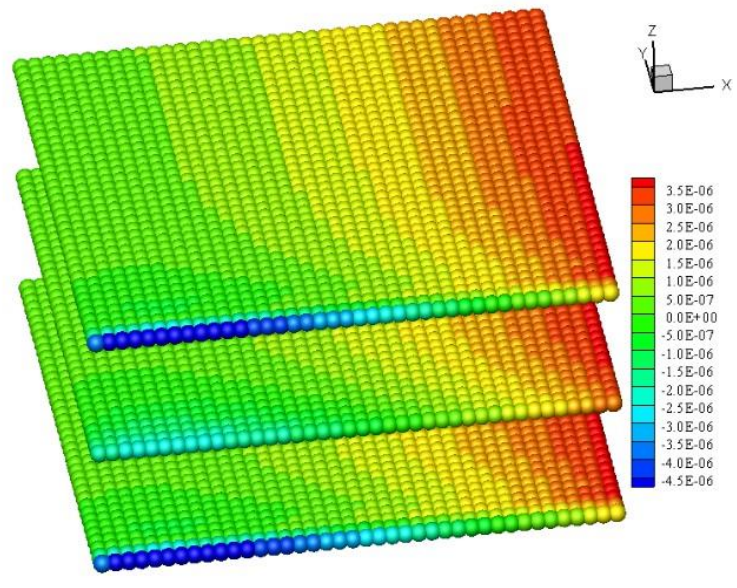

(b) $u_{y}^{*}$

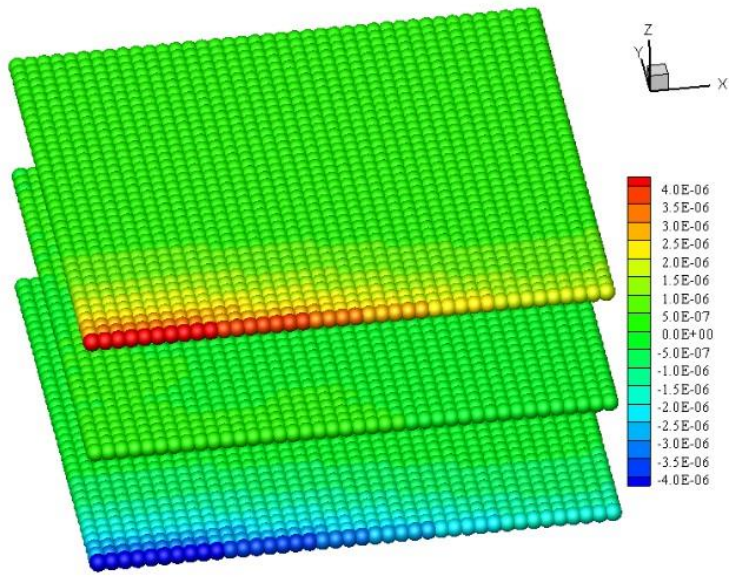

(c) $u_{z}^{*}$

Fig. 32 Non-dimensional displacement distribution at $t^{*}=0.1$ 


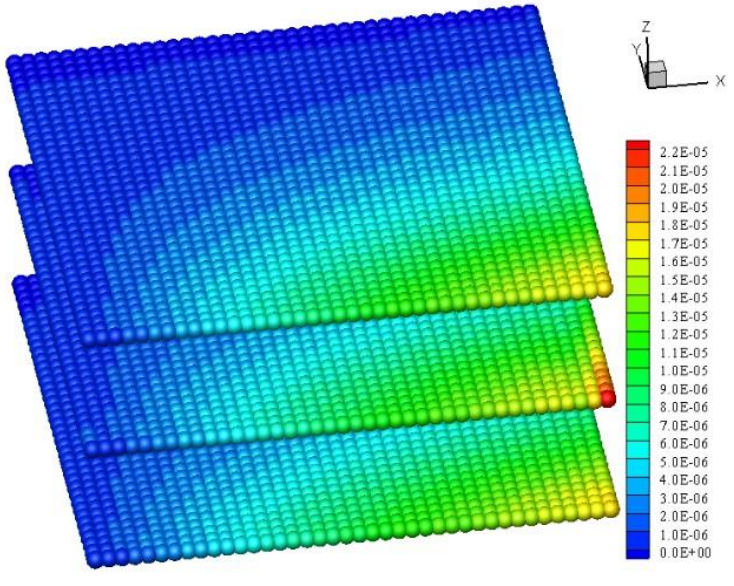

(a) $u_{x}^{*}$

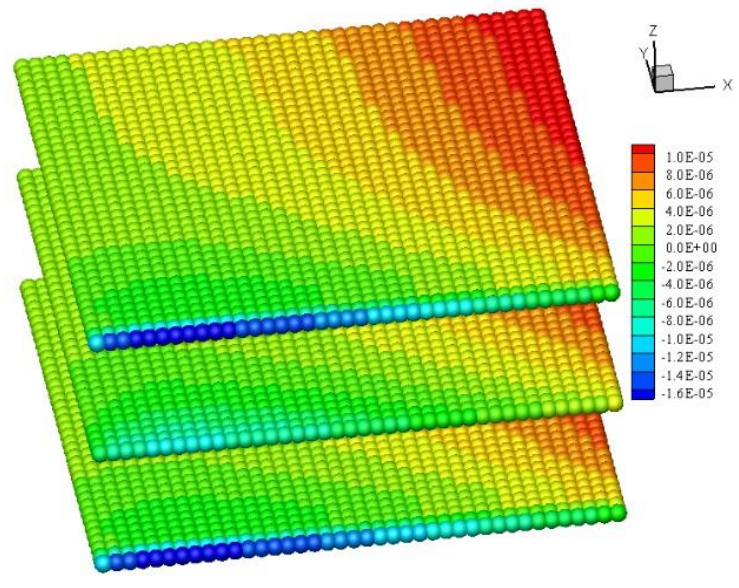

(b) $u_{y}^{*}$

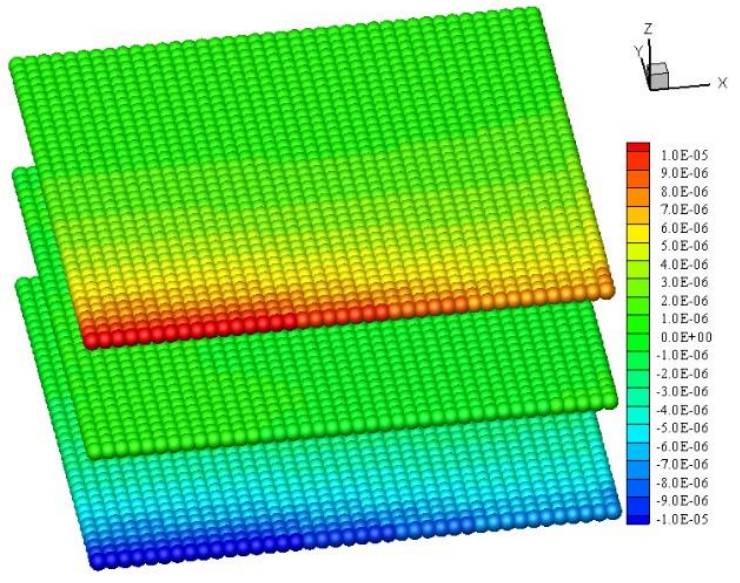

(c) $u_{z}^{*}$

Fig. 33 Non-dimensional displacement distribution at $t^{*}=0.25$

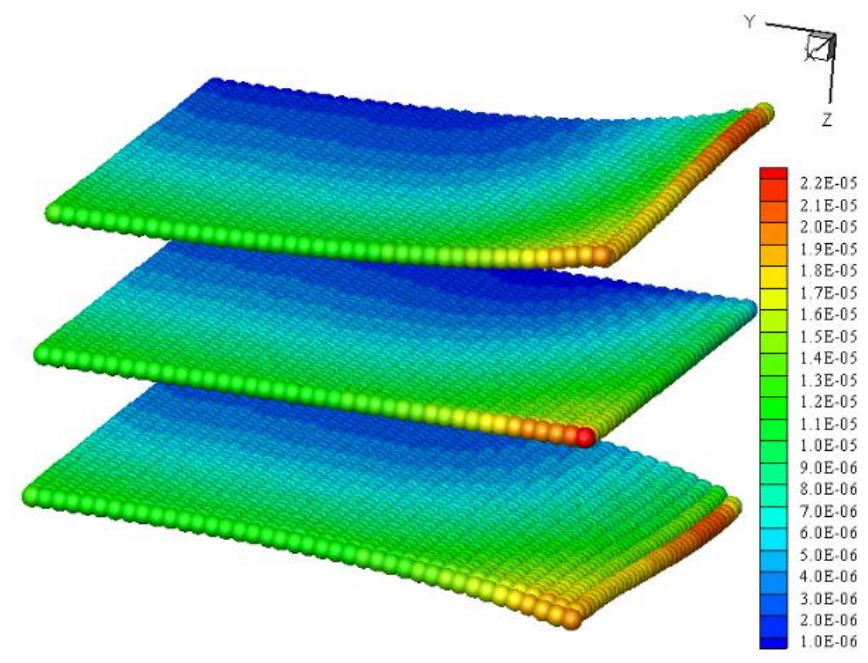

Fig. 34 Deformed shape at $t^{*}=0.25$ (displacement scale factor 200 for deformed shape) 


\section{Conclusion}

In this study, the developed PD composite model and thermo-fluid model are coupled together to predict the composite response under the fire scenario. The thermal degradations of composite material properties are incorporated into the peridynamic composite model. The fluid model coupled with heat transfer is utilized to represent the physical fire model. The ISO standard temperature-time curve is adopted to represent the fire-induced high temperature.

Two problems are investigated in the present study, i.e. composite damage due to direct contact with fire and composite damage due to hot gas induced by fire. The convective and radiative boundary conditions are implemented by coupling the thermo-fluid model and the composite model.

\section{Acknowledgement}

The authors gratefully acknowledge financial support from the China Scholarship Council (CSC No. 201506230126) and the University of Strathclyde.

\section{Data availability}

The raw/processed data required to reproduce these findings cannot be shared at this time as the data also forms part of an ongoing study.

\section{Declaration of competing interest}

The authors declare that they have no known competing financial interests or personal relationships that could have appeared to influence the work reported in this paper.

\section{Reference}

[1] Mouritz AP, Gibson AG. Fire properties of polymer composite materials: Springer Science \& Business Media, 2007.

[2] Cuoci A, Frassoldati A, Faravelli T, Ranzi E. Numerical Modeling of Laminar Flames with Detailed Kinetics Based on the Operator-Splitting Method. Energy \& Fuels. 2013;27:7730-53.

[3] van't Hof B. Numerical aspects of laminar flame simulation: Technische Universiteit Eindhoven, 1998. 
[4] Turns SR. An introduction to combustion: McGraw-hill New York, 1996.

[5] Pierce CD, Moin P. Progress-variable approach for large-eddy simulation of nonpremixed turbulent combustion. Journal of Fluid Mechanics. 2004;504:73-97.

[6] Amantini G, Frank JH, Smooke MD, Gomez A. Computational and experimental study of steady axisymmetric non-premixed methane counterflow flames. Combustion Theory and Modelling. 2007;11:47-72.

[7] Kee RJ, Rupley FM, Meeks E, Miller JA. CHEMKIN-III: A FORTRAN chemical kinetics package for the analysis of gas-phase chemical and plasma kinetics. Sandia National Labs., Livermore, CA (United States); 1996.

[8] Goodwin DG, Moffat HK, Speth RL. Cantera: An object-oriented software toolkit for chemical kinetics, thermodynamics, and transport processes. Caltech, Pasadena, CA. 2009.

[9] RConsul C, Claramunt K, Cadafalch J, Oliva AJm. Detailed numerical simulation of laminar flames by a parallel multiblock algorithm using loosely coupled computers. 2003;5:10.

[10] Coffee T, Heimerl J. Transport algorithms for premixed, laminar steady-state flames. Combustion and Flame. 1981;43:273-89.

[11] Boonkkamp TJLnotJBcoc-A, Eindhoven University of Technology, Department of Mechanical Engineering. Numerical modelling of laminar flames: a concise introduction. 2003:55-69.

[12] Manninen M, Taivassalo V, Kallio S. On the mixture model for multiphase flow. Technical Research Centre of Finland Finland; 1996.

[13] De Ris JN. Spread of a laminar diffusion flame. Symposium (International) on Combustion. 1969;12:241-52.

[14] Becker R, Braack M, Rannacher R. Numerical simulation of laminar flames at low Mach number by adaptive finite elements: Taylor \& Francis, 1999.

[15] RConsul C, Claramunt K, Cadafalch J, Oliva A. Detailed numerical simulation of laminar flames by a parallel multiblock algorithm using loosely coupled computers. Methods. 2003;5:10.

[16] ten Thije Boonkkamp J, Schreel K. Numerical modelling of laminar flames: a concise introduction. Lecture notes of the JM Burgerscentrum course on combustion: 19-22 April 2005, Eindhoven University of Technology, Department of Mechanical Engineering. 2003:55-69.

[17] Chaniotis AK, Frouzakis CE, Lee JC, Tomboulides AG, Poulikakos D, Boulouchos K. Remeshed smoothed particle hydrodynamics for the simulation of laminar chemically reactive flows. Journal of Computational Physics. 2003;191:1-17.

[18] Krishnan A, Ghoniem AF. Simulation of rollup and mixing in rayleigh-taylor flow using the transport-element method. Journal of Computational Physics. 1992;99:1-27.

[19] Soteriou MC, Ghoniem AF. Effects of the free - stream density ratio on free and forced spatially developing shear layers. Physics of Fluids. 1995;7:2036-51.

[20] Brown AL. Impact and Fire Modeling Considerations Employing SPH Coupling to a Dilute Spray Fire Code. ASME 2009 Heat Transfer Summer Conference collocated with the InterPACK09 and 3rd Energy Sustainability Conferences2009. p. 161-71.

[21] Dimitrienko YI. Thermomechanical behaviour of composite materials and structures under high temperatures: 1. Materials. Composites Part A: Applied Science and Manufacturing. 1997;28:453-61.

[22] Hopkins D, Quintiere JG. Material fire properties and predictions for thermoplastics. Fire Safety Journal. 1996;26:241-68.

[23] Gibson AG, Wright PNH, Wu YS, Mouritz AP, Mathys Z, Gardiner CP. The Integrity of Polymer Composites during and after Fire. Journal of Composite Materials. 2016;38:1283307. 
[24] Kim NK, Bhattacharyya D. Development of fire resistant wool polymer composites: Mechanical performance and fire simulation with design perspectives. Materials \& Design. 2016;106:391-403.

[25] Feih S, Mathys Z, Gibson AG, Mouritz AP. Modeling Compressive Skin Failure of Sandwich Composites in Fire. Journal of Sandwich Structures \& Materials. 2008;10:217-45.

[26] Mouritz AP, Feih S, Kandare E, Mathys Z, Gibson AG, Des Jardin PE, et al. Review of fire structural modelling of polymer composites. Composites Part A: Applied Science and Manufacturing. 2009;40:1800-14.

[27] Milke JA, Vizzini AJ. Thermal Response of Fire-Exposed Composites. Journal of Composites Technology \& Research. 1991;13:145-51.

[28] Sullivan R, Salamon N. A finite element method for the thermochemical decomposition of polymeric materials - I. Theory. International Journal of Engineering Science. 1992;30:43141.

[29] Mityushev V, Obnosov YU, Pesetskaya E, Rogosin S. Analytical Methods for Heat Conduction in Composites. Mathematical Modelling and Analysis. 2008;13:67-78.

[30] Luo C, Desjardin P. Thermo-mechanical damage modeling of a glass-phenolic composite material. Composites Science and Technology. 2007;67:1475-88.

[31] Mcmanus HL, Springer GSJJoCM. High temperature thermomechanical behavior of carbon-phenolic and carbon-carbon composites, i. analysis. 1992;26:206-29.

[32] Henderson JB, Wiebelt JA, Tant MR. A Model for the Thermal Response of Polymer Composite Materials with Experimental Verification. Journal of Composite Materials. 2016;19:579-95.

[33] Gibson AG, Wu YS, Evans JT, Mouritz AP. Laminate Theory Analysis of Composites under Load in Fire. Journal of Composite Materials. 2005;40:639-58.

[34] Silling SA. Reformulation of elasticity theory for discontinuities and long-range forces. Journal of the Mechanics and Physics of Solids. 2000;48:175-209.

[35] Silling SA, Askari E. A meshfree method based on the peridynamic model of solid mechanics. Computers \& Structures. 2005;83:1526-35.

[36] Oterkus E, Madenci E. Peridynamics for Failure Prediction in Composites. 53rd AIAA/ASME/ASCE/AHS/ASC Structures, Structural Dynamics and Materials Conference: American Institute of Aeronautics and Astronautics; 2012.

[37] Diyaroglu C. Peridynamics and its applications in marine structures [Doctor of Philosophy]. Glasgow: University of Strathclyde, 2016.

[38] Oterkus E, Madenci E. Peridynamics for failure prediction in composites. 53rd AIAA/ASME/ASCE/AHS/ASC Structures, Structural Dynamics and Materials Conference 20th AIAA/ASME/AHS Adaptive Structures Conference 14th AIAA2012. p. 1692.

[39] Oterkus E, Madenci E. Peridynamic Theory for Damage Initiation and Growth in Composite Laminate. Key Engineering Materials. 2011;488-489:355-8.

[40] Oterkus E, Barut A, Madenci E. Damage growth prediction from loaded composite fastener holes by using peridynamic theory. 51st AIAA/ASME/ASCE/AHS/ASC Structures, Structural Dynamics, and Materials Conference 18th AIAA/ASME/AHS Adaptive Structures Conference 12th2010. p. 3026.

[41] Mehrmashhadi J, Chen Z, Zhao J, Bobaru F. A stochastically homogenized peridynamic model for intraply fracture in fiber-reinforced composites. Composites Science and Technology. 2019;182:107770.

[42] Hu W, Ha YD, Bobaru F. Peridynamic model for dynamic fracture in unidirectional fiber-reinforced composites. Computer Methods in Applied Mechanics and Engineering. 2012;217-220:247-61.

[43] Xu J, Askari A, Weckner O, Silling S. Peridynamic analysis of impact damage in composite laminates. Journal of Aerospace Engineering. 2008;21:187-94. 
[44] Oterkus E, Madenci E, Weckner O, Silling S, Bogert P, Tessler A. Combined finite element and peridynamic analyses for predicting failure in a stiffened composite curved panel with a central slot. Composite Structures. 2012;94:839-50.

[45] Askari E, Xu J, Silling S. Peridynamic analysis of damage and failure in composites. 44th AIAA aerospace sciences meeting and exhibit2006. p. 88.

[46] Colavito K, Barut A, Madenci E, Phan N. Residual strength of composite laminates with a hole by using peridynamic theory. 54th AIAA/ASME/ASCE/AHS/ASC Structures, Structural Dynamics, and Materials Conference2013. p. 1761.

[47] Madenci E, Oterkus E. Peridynamic theory and its applications: Springer, 2014.

[48] Oterkus E, Madenci E. Peridynamic analysis of fiber-reinforced composite materials. Journal of Mechanics of Materials and Structures. 2012;7:45-84.

[49] Diyaroglu C, Oterkus E, Madenci E, Rabczuk T, Siddiq A. Peridynamic modeling of composite laminates under explosive loading. Composite Structures. 2016;144:14-23.

[50] Oterkus S, Madenci E. Peridynamic modeling of fuel pellet cracking. Engineering Fracture Mechanics. 2017;176:23-37.

[51] Nguyen CT, Oterkus S. Peridynamics for the thermomechanical behavior of shell structures. Engineering Fracture Mechanics. 2019;219:106623.

[52] Wang H, Oterkus E, Oterkus S. Three-Dimensional Peridynamic Model for Predicting Fracture Evolution during the Lithiation Process. Energies. 2018;11:1461.

[53] Kilic B, Madenci E. Prediction of crack paths in a quenched glass plate by using peridynamic theory. International Journal of Fracture. 2009;156:165-77.

[54] Gao Y, Oterkus S. Fully coupled thermomechanical analysis of laminated composites by using ordinary state based peridynamic theory. Composite Structures. 2019;207:397-424.

[55] Oterkus S, Madenci E. Fully coupled thermomechanical analysis of fiber reinforced composites using peridynamics. 55th AIAA/ASMe/ASCE/AHS/SC Structures, Structural Dynamics, and Materials Conference-SciTech Forum and Exposition 20142014.

[56] Madenci E, Oterkus S. Peridynamic modeling of thermo-oxidative damage evolution in a composite lamina. 58th AIAA/ASCE/AHS/ASC Structures, Structural Dynamics, and Materials Conference2017. p. 0197.

[57] Oterkus S, Madenci E, Agwai A. Peridynamic thermal diffusion. Journal of Computational Physics. 2014;265:71-96.

[58] Gao Y, Oterkus S. Peridynamic Analysis of Marine Composites under Shock Loads by Considering Thermomechanical Coupling Effects. Journal of Marine Science and Engineering. 2018;6:38.

[59] De Meo D, Oterkus E. Finite element implementation of a peridynamic pitting corrosion damage model. Ocean Engineering. 2017;135:76-83.

[60] De Meo D, Russo L, Oterkus E. Modeling of the onset, propagation, and interaction of multiple cracks generated from corrosion pits by using peridynamics. Journal of Engineering Materials and Technology. 2017;139.

[61] Jafarzadeh S, Chen Z, Bobaru F. Peridynamic modeling of repassivation in pitting corrosion of stainless steel. Corrosion. 2018;74:393-414.

[62] Jafarzadeh S, Chen Z, Bobaru F. Peridynamic modeling of intergranular corrosion damage. Journal of The Electrochemical Society. 2018;165:C362-C74.

[63] Oterkus E. Peridynamic theory for modeling three-dimensional damage growth in metallic and composite structures: University of Arizona, 2010.

[64] Le QV, Chan WK, Schwartz J. A two-dimensional ordinary, state-based peridynamic model for linearly elastic solids. International journal for numerical methods in engineering. 2014;98:547-61. 
[65] Madenci E, Oterkus S. Ordinary state-based peridynamics for plastic deformation according to von Mises yield criteria with isotropic hardening. Journal of the Mechanics and Physics of Solids. 2016;86:192-219.

[66] Madenci E, Oterkus S. Ordinary state-based peridynamics for thermoviscoelastic deformation. Engineering Fracture Mechanics. 2017;175:31-45.

[67] Imachi M, Tanaka S, Bui TQ, Oterkus S, Oterkus E. A computational approach based on ordinary state-based peridynamics with new transition bond for dynamic fracture analysis. Engineering Fracture Mechanics. 2019;206:359-74.

[68] Nguyen CT, Oterkus S. Ordinary state-based peridynamic model for geometrically nonlinear analysis. Engineering Fracture Mechanics. 2020;224:106750.

[69] Warren TL, Silling SA, Askari A, Weckner O, Epton MA, Xu J. A non-ordinary statebased peridynamic method to model solid material deformation and fracture. International Journal of Solids and Structures. 2009;46:1186-95.

[70] Breitenfeld MS, Geubelle PH, Weckner O, Silling SA. Non-ordinary state-based peridynamic analysis of stationary crack problems. Computer Methods in Applied Mechanics and Engineering. 2014;272:233-50.

[71] Amani J, Oterkus E, Areias P, Zi G, Nguyen-Thoi T, Rabczuk T. A non-ordinary statebased peridynamics formulation for thermoplastic fracture. International Journal of Impact Engineering. 2016;87:83-94.

[72] Hattori G, Trevelyan J, Coombs WM. A non-ordinary state-based peridynamics framework for anisotropic materials. Computer Methods in Applied Mechanics and Engineering. 2018;339:416-42.

[73] Wang B, Oterkus S, Oterkus E. Determination of horizon size in state-based peridynamics. Continuum Mechanics and Thermodynamics. 2020.

[74] Madenci E, Barut A, Futch M. Peridynamic differential operator and its applications. Computer Methods in Applied Mechanics and Engineering. 2016;304:408-51.

[75] Madenci E, Barut A, Dorduncu M. Peridynamic differential operator for numerical analysis: Springer, 2019.

[76] Madenci E, Dorduncu M, Gu X. Peridynamic least squares minimization. Computer Methods in Applied Mechanics and Engineering. 2019;348:846-74.

[77] Gao Y, Oterkus S. Non-local modeling for fluid flow coupled with heat transfer by using peridynamic differential operator. Engineering Analysis with Boundary Elements. 2019;105:104-21.

[78] Gao Y, Oterkus S. Nonlocal numerical simulation of low Reynolds number laminar fluid motion by using peridynamic differential operator. Ocean Engineering. 2019;179:135-58.

[79] Henderson JB, Wiebelt JA, Tant MR. A Model for the Thermal Response of Polymer Composite Materials with Experimental Verification. Journal of Composite Materials. 1985;19:579-95.

[80] ISO IIOfS, Geneva, Switzerland. 834: Fire resistance tests-elements of building construction. 1999.

[81] Ou KS, Zheng J, Luo WJ, Li XF, Hu HS, Yang JB, et al. Heat and Mechanical Response Analysis of Composite Compressed Natural Gas Cylinders at Vehicle Fire Scenario. Procedia Engineering. 2015;130:1425-40.

[82] Gibson A, Browne T, Feih S, Mouritz A. Modeling composite high temperature behavior and fire response under load. Journal of Composite Materials. 2012;46:2005-22.

[83] Huang X, Gillespie JW, Eduljee RF. Effect of temperature on the transverse cracking behavior of cross-ply composite laminates. Composites Part B: Engineering. 1997;28:419-24.

[84] Kaw AK. Mechanics of composite materials: CRC press, 2005.

[85] Rahmani H, Najafi SHM, Ashori A. Mechanical performance of epoxy/carbon fiber laminated composites. Journal of Reinforced Plastics and Composites. 2014;33:733-40. 
[86] Gibson AG, Browne TNA, Feih S, Mouritz AP. Modeling composite high temperature behavior and fire response under load. Journal of Composite Materials. 2012;46:2005-22.

[87] Gray DD, Giorgini A. The validity of the boussinesq approximation for liquids and gases. International Journal of Heat and Mass Transfer. 1976;19:545-51.

[88] Merrikh AA, Lage JL. Natural convection in an enclosure with disconnected and conducting solid blocks. International Journal of Heat and Mass Transfer. 2005;48:1361-72.

[89] Szewc K, Pozorski J, Tanière A. Modeling of natural convection with Smoothed Particle Hydrodynamics: Non-Boussinesq formulation. International Journal of Heat and Mass Transfer. 2011;54:4807-16.

[90] Batchelor GK. An introduction to fluid dynamics: Cambridge university press, 2000.

[91] White FM, Corfield I. Viscous fluid flow: McGraw-Hill New York, 2006.

[92] Chenoweth DR, Paolucci S. Natural convection in an enclosed vertical air layer with large horizontal temperature differences. Journal of Fluid Mechanics. 2006;169:173-210.

[93] Luo C, Xie W, DesJardin PE. Fluid-Structure Simulations of Composite Material Response for Fire Environments. Fire technology. 2009;47:887-912.

[94] Intel R. Intel math kernel library reference manual. Tech. Rep. 630813-051US, 2012.[Online]. Available: http://software. intel. $\mathrm{com} / \mathrm{sites} / \mathrm{products} /$ documentation/hpc/mkl/mklman/mklman. pdf; 2011.

[95] Oterkus S. Peridynamics for the solution of multiphysics problems [Doctor of Philosophy]. Arizona: The University of Arizona, 2015.

[96] McGrattan KB, Baum HR, Rehm RG, Hamins A, Forney GP. Fire dynamics simulator-Technical reference guide: National Institute of Standards and Technology, Building and Fire Research, 2000.

[97] Kilic B, Madenci E. An adaptive dynamic relaxation method for quasi-static simulations using the peridynamic theory. Theoretical and Applied Fracture Mechanics. 2010;53:194-204.

[98] Monaghan JJ. Simulating Free Surface Flows with SPH. Journal of Computational Physics. 1994;110:399-406.

[99] Elder JW. Turbulent free convection in a vertical slot. Journal of Fluid Mechanics. 2006;23:99-\&. 